\title{
Characterization and valorization of humins produced by HMF degradation in ionic liquids: A valuable carbonaceous material for antimony removal
}

\author{
Amir Al Ghatta, ${ }^{\star a, b}$ Xinyi Zhou, ${ }^{a, b}$ Giulia Casarano,, ${ }^{b}$ James D. E. T. Wilton-Ely, ${ }^{\star b}$ Jason P. Hallett*a \\ a Department of Chemical Engineering, Imperial College London, Imperial College Road, South \\ Kensington Campus, London SW7 2AZ (UK) \\ b Department of Chemistry, Imperial College London, Molecular Sciences Research Hub, White City \\ Campus, 82 Wood Lane, London W12 0BZ (UK) \\ E-mail: a.al-ghatta16@imperial.ac.uk \\ E-mail: j.wilton-ely@imperial.ac.uk \\ E-mail: j.hallett@imperial.ac.uk
}

\begin{abstract}
The processing of biomass in ionic liquids has demonstrated many benefits compared to organic solvents. This includes the maximization of 5-hydroxymethylfurfural (HMF) yield from sugars through the suppression of byproducts, such as formic acid and levulinic acid. Inefficiencies still exist due to the low stability of HMF at high temperature, leading to side reactions which ultimately result in the undesirable formation of humins. Valorization of this polymeric side product is thus needed to improve the economics of the biorefinery and could lead to humins being viewed as valuable materials for various applications. However, a much better understanding is needed of how humins form from HMF in the various ionic liquids proposed for the biorefinery. In this contribution, humin formation is probed by a range of analytical techniques, including FT-IR, SEM, solid state ${ }^{13} \mathrm{C}$ NMR, MS, GPC and XPS. This reveals that the structure and morphology of the humins formed does not resemble those reported in the literature and the material displays a number of unique aspects. The hydrogen bonding proprieties of the ionic liquids employed exert a strong influence on the chemical functionality of the humins and this is used to demonstrate their potential as functional materials. To demonstrate this, the humins produced in various ionic liquid environments are applied to metal extraction and compared with commercial activated carbon. This revealed that humins are superior for the extraction of antimony ions from waste water, showing promise as an adsorbent additive for water purification.
\end{abstract}

Keywords: ionic liquids, HMF, biorefinery, carbonaceous materials, antimony

\section{INTRODUCTION}

The valorization of sugars to valuable chemical building blocks is a key step in the implementation of a successful biorefinery within a modern economic setting. ${ }^{1}$ Various processes have been explored which are aimed at the efficient synthesis of the versatile biorefinery product 5-hydroxymethylfurfural (HMF), which is the starting point for the synthesis of a large variety of platform chemicals. ${ }^{2}$ Important results have been achieved using ionic liquids solvents, which have led to remarkably improved yields from fructose and permitted the exploitation of cheaper feedstocks, such as glucose and cellulose. ${ }^{3}$ However, HMF is unstable at high temperatures in the ionic liquid medium, mainly due to the reactivity of the hydroxyl group, which is also the origin of the challenges surrounding its isolation. ${ }^{4}$ Galkin and coworkers have highlighted the instability of HMF at room temperature in terms of its physical state. Since the melting point of this compound is between $30-34{ }^{\circ} \mathrm{C}$, it can exist as a solid or in a metastable liquid state. The liquid state undergoes faster degradation due to the increased diffusion of the molecules and residual acidity from the synthetic pathway. ${ }^{5}$ For this reason, the same authors proposed 5methylfurfural as an alternative biorefinery target product. ${ }^{6}$ Recently, it has also been reported that the conversion of HMF to 2,5-diformylfuran (DFF) in ionic liquids at low temperature represents a valuable option to overcome the separation and stability issues related to HMF, since DFF is stable at room temperature and can be separated from the ionic liquid by sublimation. ${ }^{7}$ The in situ conversion of HMF 
into other more valuable chemicals is a promising approach with which to overcome the issues associated with its separation. However, this does not avoid the formation of humins and their reactive functionality leads to the deactivation of many catalysts, limiting the catalyst options available. For example, it is reported that most ruthenium-based catalysts are inactive for the oxidation of HMF in ionic liquids, forming humins instead. ${ }^{8}$ Yan and co-workers ${ }^{9,10}$ developed a catalyst based on iron and zirconium oxides to convert HMF to 2,5-furandicarboxylic acid (FDCA) in [bmim]Cl (bmim = 1-butyl-3methylimidazolium), which showed good tolerance of humins but displayed low selectivity. A recent report describes the same reaction in the same solvent using polyoxometallate catalysts for the highyield synthesis of FDCA. ${ }^{11}$ However, all catalytic systems reported to date are adversely affected by the formation of humins, necessitating the use of prohibitively low substrate concentrations in order to suppress the side reactions that produce them. This is a significant drawback as high substrate loadings are needed to facilitate separation of the products and to make the process economically viable. ${ }^{4}$

These challenges are not limited to ionic liquids, as issues related to the formation of humins exist for all solvents used to convert sugars into a value-added products. Therefore, this aspect represents one of the main barriers to obtaining high yields in these processes. In order to establish a complete and techno-economically efficient biorefinery that adheres to the principles of green chemistry, ${ }^{12}$ the characterization and valorization of all major byproducts is needed. To this end, various studies have focused on the characterization of humins formed with water as the solvent. These investigations aimed to produce a highly functionalized bio-derived material and led to various proposals regarding the mechanism of formation and the chemical structure of the humins formed. Hoang and coworkers analyzed glucose-derived humins produced under strongly acidic conditions and demonstrated their application in syngas production. The authors showed that humins grow as spherical particles, in which the morphology and chemical composition changes upon heating, passing from an amorphous furanic material to a benzene-based framework. ${ }^{13}$ Other studies have focused solely on the characterization of humins formed under different conditions without exploring any specific application. Through the analysis of the amounts of different functional groups observed, Patil and Lund showed that different morphologies of humins are formed on addition of an acid or by thermal decomposition. ${ }^{14}$ Acid-catalyzed generation of humins results in the formation of spheres that grow with increasing reaction time. ${ }^{13}$ In both acid and thermally-driven processes, the absence of aldehyde groups in the IR spectra indicate that aldol condensation is the main mechanism involved in the polymerization. A similar study was performed by Cheng and co-workers, who investigated the soluble fractions of humins in different organic solvents using LC-MS, showing that all soluble fractions have the same molecular structure and that solubility in water depends exclusively on molecular weight. ${ }^{15}$

While current theory supports the observation that ionic liquids stabilize HMF towards overdehydration into formic and levulinic acids, other side reactions occur due to the reaction of sugars with the HMF produced and the thermal instability of HMF at high temperatures.,16,17 Different studies have observed that the thermal degradation of HMF in the ionic liquid is constant, but this process is partially suppressed when a Lewis acid is present (at low temperature). ${ }^{18,19}$ The reason for this is unclear. At temperatures higher than $180{ }^{\circ} \mathrm{C}$, it has been reported that cross polymerization takes place between the $[\mathrm{bmim}]^{+}$cation of the ionic liquid and the aldehyde group of HMF due to the generation of a carbene through deprotonation of the $\mathrm{C} 2$ position on the imidazolium ring. ${ }^{20}$ 
While most reports have studied the humins derived from acid dehydration of sugars, the work described here will focus on the stability of HMF in ionic liquids and the analysis of humins as the products of HMF degradation. The motivation for this study lies in the potential of the ionic liquid medium to act as a template for the synthesis of functional materials with high porosity and specific additional properties. ${ }^{21}$ Antonietti and co-workers used [emim] [DCA] (emim = 1-ethyl-3-methylimidazolium; DCA = dicyanamide) to synthesize a nitrogen-doped, carbon-based graphitic material which displayed high conductivity. It was found that using ionic liquids as nitrogen precursors guarantees a homogenous distribution of nitrogen in the carbon framework. ${ }^{22}$ In another report, hierarchical porous carbonaceous materials were prepared by Titirici and co-workers via ionothermal carbonization of carbohydrates using $[$ bmim $]\left[\mathrm{FeCl}_{4}\right]$ as the reaction medium. ${ }^{23}$

The work reported here focuses on the formation of humins in various ionic liquids commonly used for biomass processing. It was found that the differing hydrogen bonding ability of the anion in the ionic liquids studied (Figure 1) not only triggers the formation of humins but also influences their morphology and chemical structure. These findings are also significant in the context of the sustained interest in carbonaceous materials for a large variety of applications. ${ }^{24}$ Herein we show that humins derived from HMF have great potential as a carbonaceous material for the extraction of antimony from waste water. This is particularly significant as the removal of this particular contaminant has been inefficient using conventional or chemically treated activated carbon. ${ }^{25,26}$ Furthermore, the mild conditions used to form the humins represent a less energy intensive process than that required for activated carbon, since no activation procedure using steam or chemical treatment is required.
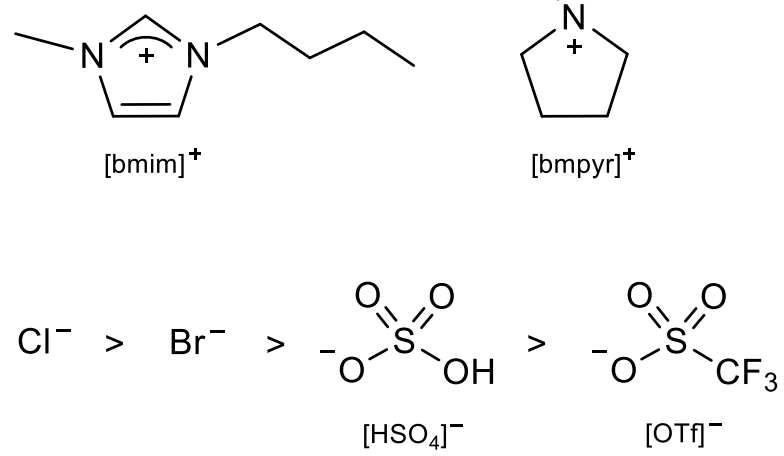

Figure 1. The ionic liquids used in the study with anions ordered by hydrogen bonding ability.

\section{EXPERIMENTAL SECTION}

\section{Materials}

The ionic liquids were prepared according to the procedures reported in the literature. ${ }^{16}$ Further details are provided in the Supporting Information.

\section{HMF stability}

Mixtures of ionic liquids (ILs) and HMF were prepared in large batches using the following procedure. The ionic liquids $(3 \mathrm{~g})$ and $\operatorname{HMF}(300 \mathrm{mg})$ were mixed at the desired mass ratio (1:10) followed by 
addition of a known amount of organic solvent $(10 \mathrm{~mL})$ to solubilize both the HMF and the ionic liquid. For this purpose, dichloromethane was chosen for [bmim] Cl, [bmim]Br and [bmim][OTf], while methanol was selected for [bmim] $\left[\mathrm{HSO}_{4}\right]$ since this $\mathrm{IL}$ is insoluble in dichloromethane. Aliquots of these mixtures were placed in a $3 \mathrm{~mL}$ vial followed by complete solvent removal under vacuum (overnight). The reaction was initiated by placing the vial on a preheated heating plate at the selected temperature and left for the desired time. The reaction was then quenched with water $(3 \mathrm{~mL})$, filtered and the residual HMF measured by HPLC. This analysis was performed using a Shimadzu Prominence preparative HPLC (Shimadzu, Kyoto, Japan) with refractive index and ultraviolet (UV) detectors. The instrument was equipped with a C18 Agilent reverse phase column using methanol/water (1:9) as the mobile phase with a flow rate of $0.6 \mathrm{~mL} / \mathrm{min}$ at $40{ }^{\circ} \mathrm{C}$. Degradation $(z)$ was calculated according to Equation 1 where $C_{i}$ and $C_{f}$ are the initial and final concentrations of HMF in solution:

Eq. 1.

$$
z=\frac{C_{i}-C_{f}}{C_{i}}
$$

\section{Synthesis of humins}

The HMF (300 mg) and ionic liquids $(1 \mathrm{~g})$ were mixed in a $10 \mathrm{~mL}$ vial and placed on a preheated heating block $\left(160^{\circ} \mathrm{C}\right)$ for the 6 hours. Water was added to quench the reaction and precipitate the humins, which were separated by centrifugation and washed with water until the liquid phase became clear. For [bmim] $\left[\mathrm{HSO}_{4}\right]$, the washing was repeated until the $\mathrm{pH}$ of the washings became neutral. The humins were then dried in an oven at $60^{\circ} \mathrm{C}$ overnight. In order to enhance the production of humins, the ionic liquid was recovered as a solution in water (by filtration). The water was removed at low pressure, further HMF was added and the cycle repeated under the same conditions until an appreciable amount of humins (> $200 \mathrm{mg}$ ) had formed, as shown in Figure 2.

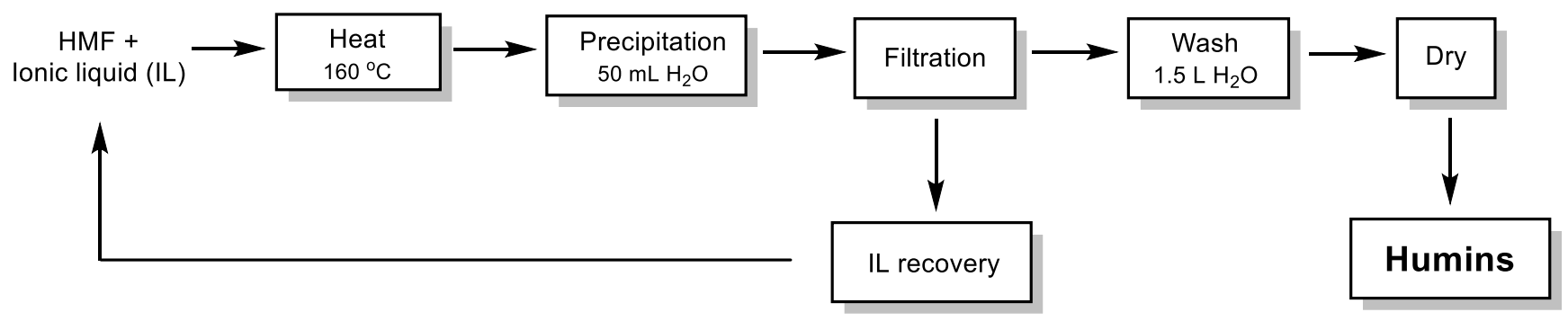

Figure 2. Synthesis of humins in ionic liquids.

The goal of this investigation was to produce sufficient humins for characterization and investigation of their properties. No attempt was made to optimize the degradation of HMF to form humins, as the premise of this paper is that they would be formed in sufficient amounts as an undesirable byproduct of future biorefineries.

\section{Characterization of humins}

For GPC analysis, samples were prepared by dissolving $20 \mathrm{mg}$ of the humins in $1 \mathrm{~mL}$ GPC grade DMSO containing $\operatorname{LiBr}\left(1 \mathrm{~g} \cdot \mathrm{L}^{-1}\right)$ and filtered through a $0.2 \mu \mathrm{m}$ syringe filter. GPC measurements were performed using an Agilent 1260 Infinity instrument equipped with a Viscotek column set (AGuard, A6000 M and 
A3000 M) and using GPC grade DMSO as eluent at a flow rate of $0.4 \mathrm{~mL} \cdot \mathrm{min}^{-1}$ at $60{ }^{\circ} \mathrm{C}$. An Agilent 1260 Infinity RID detector was used for detection. Calibration of the instrument was conducted using 10 pullulan standards (Agilent calibration kit, $180<$ molecular weight $<780,000$ ). Mass spectrometry was performed using a Waters LCT Premier (ES-ToF) Acquity i-Class instrument.

XPS analysis was performed using a Thermo NEXSA XPS instrument fitted with a monochromated Al ka X-ray source (1486.7 eV), a spherical sector analyzer, a 3 multichannel resistive plate and 128 channel delay line detectors. All data were recorded at $19.2 \mathrm{~W}$ and an X-ray beam size of $200 \times 100 \mu \mathrm{m}$. Survey scans were recorded at a pass energy of $160 \mathrm{eV}$ and high-resolution scans recorded at a pass energy of $20 \mathrm{eV}$. Electronic charge neutralization was achieved using a dual-beam low-energy electron/ion source (Thermo Scientific FG-03). The ion gun current was $150 \mu \mathrm{A}$ and the ion gun voltage was $45 \mathrm{~V}$. All sample data were recorded at a pressure below $10^{-8}$ Torr and at room temperature (294 K). XPS spectra were fitted using CasaXPS and processed using OriginPro 2017 with Savitzky-Golay smoothing over a 10 point of window for further refining. Peaks were fitted with a Shirley background prior to component analysis. Full width at half maximum (FWHM) values were allowed to vary in the range 1.3-1.9 and the peak position was chosen based on literature values. ${ }^{27}$ Spectra were referenced to the most intense carbon peak at $284.5 \mathrm{eV}$, which was identified as an $\mathrm{sp}^{2}$-like carbon.

The surface area of the humins was calculated using Brunauer-Emmett-Teller (BET) analysis with a Tristar 3000 instrument with degassing at $5^{\circ} \mathrm{C}$ with nitrogen for 1 hour.

\section{Metal extraction}

A mother solution of the metals as nitrate salts (100 ppm) was prepared by dilution of an ICP standard solution of trivalent antimonite or divalent lead ions (1000 ppm) at $\mathrm{pH} 2$. The $\mathrm{pH}$ was then corrected to $\mathrm{pH} 4$ using a solution of $0.1 \mathrm{M} \mathrm{NaOH}$. In a typical experiment, $5 \mathrm{mg}$ of humins (or activated carbon) was mixed with $5 \mathrm{~mL}$ of metal ion solution in a $10 \mathrm{~mL}$ vial and mixed overnight (12 h) in a vortex mixer. At the end of the reaction, the solid was removed with a $0.2 \mu$ filter and diluted by a factor of 100 for ICP analysis to determine the concentration of residual metal. The extraction efficiency $(y)$ is defined using Equation 2 where $x$ refers to the initial (i) and final (f) concentrations. The experiments were carried out in triplicate.

Eq. 2.

$$
y=\frac{x_{i}-x_{f}}{x_{i}}
$$

Freundlich constants were derived by fitting the adsorbed amount of metal per unit of adsorbent ( $q$ in $\mathrm{mg} / \mathrm{g}$ ) and the initial concentration (ppm) with Equation 3:

Eq. 3.

$$
q=Q s a t * K * x_{i}^{n} /\left(1+K * x_{i}^{n}\right)
$$

Where Qsat is the saturation amount of the solid and K and $\mathrm{n}$ characteristic constants of the system which represent the affinity of the metal towards the active sites of the solid. Fitting was done using OriginPro 2019 with the integrated non-linear fitting package with an orthogonal distance regression method with $R^{2}$ of 0.99 . 


\section{RESULTS AND DISCUSSION}

\section{Synthesis of humins}

The yield of humins was found to vary in each ionic liquid tested and these data are reported in Table 1. Particularly notable was the observation that the substantial hydrogen bond acceptor ability of the chloride ions inhibited the formation of humins in high yield. This was the case even after three cycles of the synthetic protocol, indicating that strong hydrogen bonding limits the formation of humins. In contrast, weaker hydrogen bonding was found to favor the formation of humins, leading to the maximum yield being recorded when these interactions are minimized, through using the triflate (trifluoromethanesulfonate) anion. This suggests that chloride anions tend to cause degradation of HMF to form water soluble oligomers rather than producing high molecular weight polymers. Furthermore, this degradation appears not to be selective since no well-defined products are observed by HPLC analysis. Consequently, the strong coordination of the halide ions to the hydroxyl group of HMF results in negligible conversion to humins. In [bmim] $\mathrm{Br}$, only $22 \%$ of the $\mathrm{HMF}$ degradation products obtained were humins and an even lower yield of humins was obtained in [bmim] $\left[\mathrm{HSO}_{4}\right]$. In the latter case, this might be due not only to an intermediate hydrogen bonding ability, but also to an alternative degradation process, such as overhydration to levulinic and formic acid. In contrast, the triflate anion offers negligible coordinating ability, leading to more facile HMF degradation and a tendency to form humins as the degradation product.

The fact that humins were formed in substantial amounts only after the second or even third cycles suggests that initial degradation of the starting material proceeds via non-humin oligomers with low molecular weight that are soluble and do not precipitate upon addition of water. Hence, subsequent cycles would benefit from the presence of an increased concentration of such oligomers, which will aggregate and form a high mass insoluble polymer.

Table 1. Yield of humins in different ionic liquids $(1 \mathrm{~g})$ after heating HMF $(300 \mathrm{mg})$ at $160{ }^{\circ} \mathrm{C}$ for $6 \mathrm{~h}$ followed by re-use of ionic liquid with fresh HMF in a new cycle. Kamlet-Taft parameters ( $\alpha, \beta$ and $\pi$ ) taken from reference 28 or references $29^{a}$ and $30^{b}$. Yields (based on mass) are for humins isolated from each cycle.

\begin{tabular}{lccccc}
\hline lonic liquid & Cycle & Yield of humins (\%) & $\boldsymbol{\alpha}$ & $\boldsymbol{\beta}$ & $\boldsymbol{\pi}$ \\
\hline$[\mathrm{bmim}] \mathrm{Cl}$ & 1 & 0 & 0.32 & 0.95 & 1.13 \\
& 2 & 0 & & & \\
& 3 & 2 & & & \\
\hline$[\mathrm{bmim}] \mathrm{Br}$ & 1 & 0 & 0.36 & 0.87 & 1.06 \\
& 2 & 22 & & & \\
& 3 & 22 & & & \\
\hline$[$ bmim][HSO 4$]$ & 1 & 8 & $\mathrm{~N} / \mathrm{A}^{\mathrm{a}}$ & $0.67^{\mathrm{a}}$ & $1.09^{\mathrm{a}}$ \\
& 2 & 25 & & & \\
\hline$[\mathrm{bmim}][\mathrm{OTf}]$ & 1 & 22 & 0.50 & 0.57 & 0.90 \\
& 2 & 24 & & & \\
\hline$[\mathrm{bmpyr}][\mathrm{OTf}]$ & 1 & 0 & $\mathrm{~N} / \mathrm{A}^{\mathrm{b}}$ & $0.59^{\mathrm{b}}$ & $089^{\mathrm{b}}$ \\
& 2 & 65 & & & \\
& 3 & 53 & & & \\
\hline
\end{tabular}


By analyzing the reaction mixture using HPLC, the conversion of HMF was measured at different temperatures and times (Figure 3). At temperatures below $100{ }^{\circ} \mathrm{C}$, no significant degradation was observed for all the ionic liquids tested. The ionic liquid [bmim] $\left[\mathrm{HSO}_{4}\right]$ led to a greater extent of degradation of HMF compared to ionic liquids with triflate, chloride or bromide anions, probably due to the protic nature of hydrogensulfate, which can catalyze the degradation of HMF. At higher temperatures, the bromide-based ionic liquid shows a lower degree of degradation compared to ionic liquids with either chloride or triflate anions, which suggests that an optimum hydrogen bonding strength exists, favoring the stabilization of HMF. A kinetic analysis at $160{ }^{\circ} \mathrm{C}$ showed that the degradation of HMF is less influenced by hydrogen bonding at high temperature, instead showing similar trends and values for both chloride and triflate. However, the partial suppression of degradation in the ionic liquid with bromide anions suggests that hydrogen bonding retains some influence even at high temperature. Moreover, the fact that the use of an ionic liquid with a chloride anion (in contrast to triflate) fails to result in the formation of humins suggests that degradation leads mainly to the formation of low molecular weight, water-soluble oligomers, which do not aggregate to form insoluble polymers of higher molecular weight. The formation of levulinic acid and formic acid was not detected when ionic liquids with bromide, chloride or triflate anions were used to form humins and only small amounts $(<5 \%)$ were detected by HPLC when using $[\mathrm{bmim}]\left[\mathrm{HSO}_{4}\right]$.
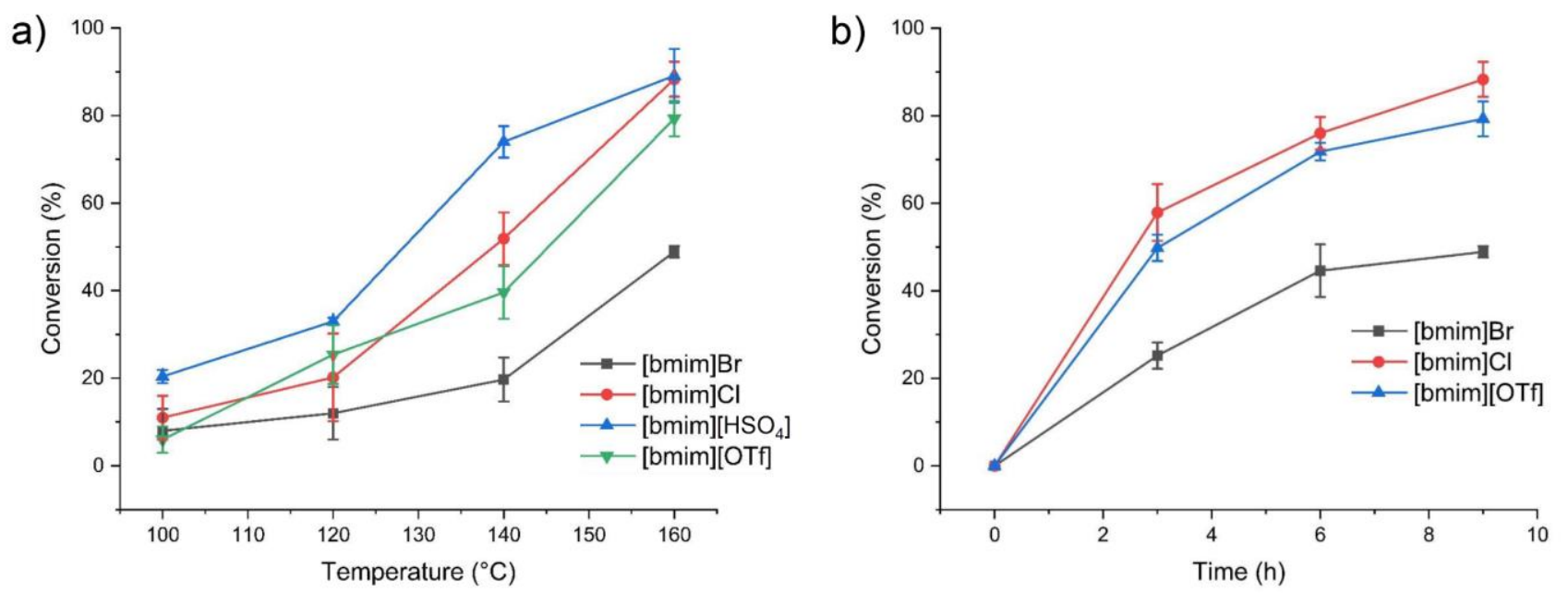

Figure 3. HMF degradation in a range of ionic liquids at a) different temperatures $(t=9 \mathrm{~h})$ and $b)$ different times $\left(\mathrm{T}=160^{\circ} \mathrm{C}\right) .10 \% \mathrm{HMF}$ loading.

\section{Analysis of humins}

In order to identify the functionality present in the different humins formed, FT-IR spectroscopy was performed on each sample and compared with the spectrum for the original substrate (Figure 4). Humins produced by ionic liquids with [bmpyr] ${ }^{+}$(1-butyl-1-methylpyrrolidinium) and [bmim] ${ }^{+}$cations and the same anion exhibited the same infrared spectra, indicating that the chemical structure of the solid material obtained in our study is dictated solely by the anion. This is consistent with studies performed on HMFionic liquid mixtures, showing that the cation does not contribute to the interactions between the ionic liquid system and HMF. In contrast, the anions can establish strong hydrogen bonds with the hydroxyl group of HMF.4,31 Indeed, HMF undergoes degradation in organic solvents with high $\beta$ value such as 
dimethylsulfoxide (DMSO) and dimethylformamide (DMF), although the extent is lower than that for [bmim] Cl, due to their lower basicity. ${ }^{32}$ It has been reported that HMF is a key species in the formation of humins during biomass conversion and that it can maintain its characteristic furanic structure even after incorporation into a polymeric network. ${ }^{33}$ Therefore, the spectra of the humins obtained under the conditions used in this study were recorded alongside the spectrum of HMF. In this way, the structural changes of the starting material upon polymerization could be monitored, as well as the influence of the solvent on the functionalization of the humins.

A number of significant differences were observed in the characterization of the humins formed from the HMF in the different ionic liquids. The absorption for the hydroxyl group of HMF in the noncoordinating triflate ionic liquid (around $3400 \mathrm{~cm}^{-1}$ ) disappears, indicating condensation of the hydroxyl group. The fact that the carbonyl signal at $1655 \mathrm{~cm}^{-1}$ remains well-defined and unshifted indicates that the mechanism involves reaction of $-\mathrm{OH}$ groups alongside the preservation of carbonyl groups in the product. The spectra of these humins show similarities to the features in the starting material with key differences between 1037-1051 $\mathrm{cm}^{-1}$ and at $886 \mathrm{~cm}^{-1}$. These bands can be attributed to the vibrations of a C-O-C linkage connecting the furan rings, as has already been reported elsewhere. ${ }^{34,35}$

The humins formed in the imidazolium-based ILs with bromide and hydrogensulfate anions exhibit the same IR spectra (Figure 4b) suggesting a similar structure. The absorption of the hydroxyl group is replaced by a broad peak with a lower intensity, which is attributed to a carbonyl moiety, indicating the formation of carboxylic acid units. The furanic signals at 755-788, 964, 1020 and $1523 \mathrm{~cm}^{-}$ ${ }^{1}$ are retained, suggesting that the main furanic network still exists but with different connectivity. The IR spectra for these types of humins are in agreement with those reported by Yan and co-workers, who identified humins as the main side product from the catalytic aerobic oxidation of HMF to FDCA. ${ }^{9}$ Furthermore, the FT-IR spectra of the humins formed in [bmim] $\left[\mathrm{HSO}_{4}\right]$ and $[\mathrm{bmim}] \mathrm{Br}$ exhibits similar features to those reported by $\mathrm{Xu}$ and co-workers during the dehydration of glucose in [bmim] Cl at high sugar concentration (50\%). The same group observed mostly oligomers at low sugar loadings ${ }^{36}$ This suggests that the origin of the formation of insoluble humins is the instability of HMF at high concentration and temperature. The peaks associated with the aldehyde and furan ring in the IR spectra at lower frequency are observed to have decreased in intensity suggesting that breakdown of the furanic structure occurs to some extent. It has already been demonstrated that sugars undergo decomposition to form a highly aromatic structure when iron ions are present to promote sugar dehydration. ${ }^{23,37}$ The hydrogensulfate anion could act as a Brønsted acid, enhancing degradation of HMF in a similar effect to the iron-based Lewis acids reported in the literature. ${ }^{38}$ 

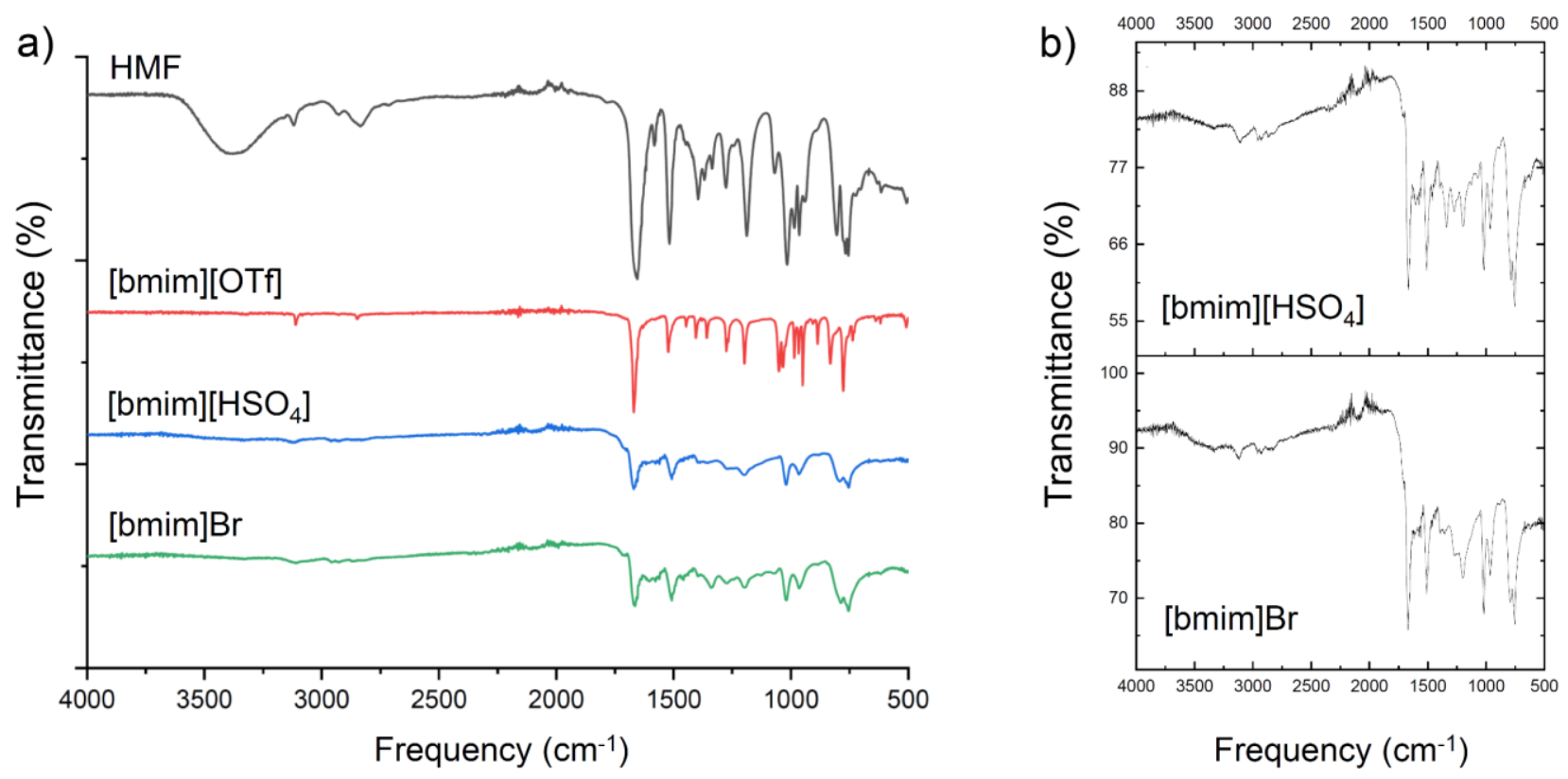

Figure 4. a) Comparison of IR spectra of HMF with humin samples obtained in different ionic liquids and b) IR spectra of humins formed in the two ionic liquids known to engage in substantial hydrogen bonding.

In order to further confirm that new ether bonds had been formed in the humins generated in the triflate ionic liquid, it was decided to analyze the components that were soluble in organic solvents. A fraction soluble in tetrahydrofuran was separated in very small quantities as a yellowish powder and analyzed. The IR spectrum of this compound was found to be the same as that for the corresponding humins, suggesting that this fraction soluble in organic solvents could be a repeating unit of the polymer with a lower molecular weight (Figure 5a). Extensive washing of the humins with tetrahydrofuran did not lead to a change in the IR spectrum. A further study was performed, resulting in two significant peaks at $m / z 235$ (HMF dimer) and $m / z 467$ in the high-resolution mass spectrum. Based on these findings, a tentative mechanism is proposed and this is shown in Figure $5 \mathrm{~b}$. In the mechanism, one aldehyde group condenses to form an acetal with two hydroxyl groups, while the residual hydroxyl group undergoes condensation with another HMF molecule. Propagation to form higher molecular weights through condensation with other HMF molecules is then possible. This leads to a structure without hydroxyl groups and correlates well with the features in the IR spectra obtained. It is possible that the tetramer unit could react with other HMF molecules or other oligomers possessing a hydroxyl group to form a higher molecular weight compound via the same mechanism. 


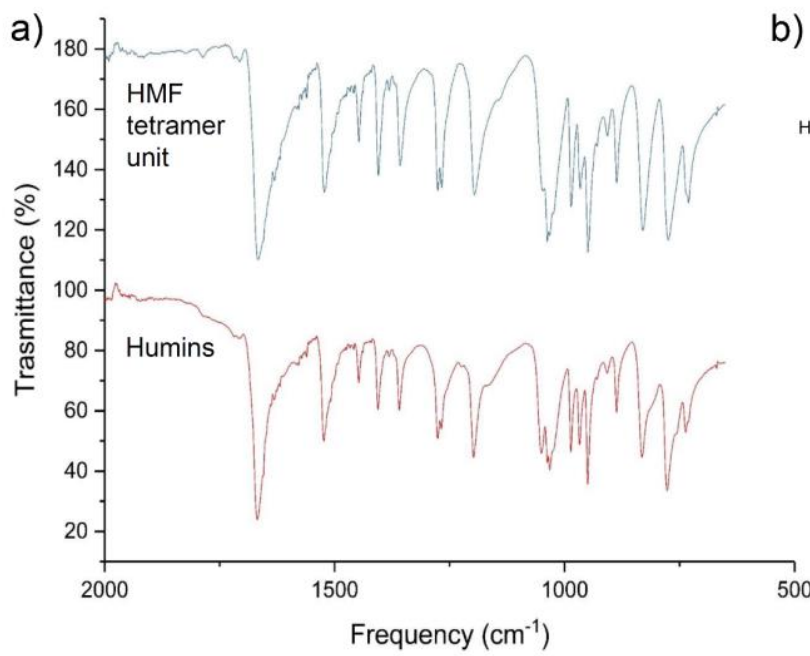

b)

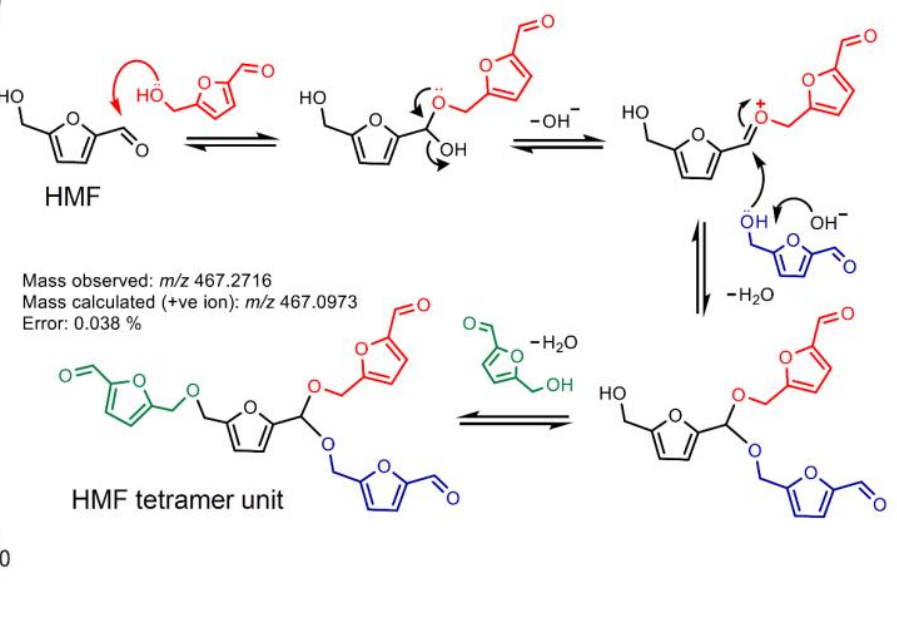

Figure 5. Analysis of material formed in [bmim][OTf] showing a) IR spectra of the isolated HMF tetramer unit and the main polymer; b) proposed mechanism of formation of the tetramer unit from HMF, in agreement with mass spectrometry analysis.

Further insight into the chemical structure was gained using solid state ${ }^{13} \mathrm{C}\left\{{ }^{1} \mathrm{H}\right\}$ NMR spectroscopy. Spectra were recorded and these are presented in Figure 6 for the humins produced in [bmim] $\left[\mathrm{HSO}_{4}\right]$ and [bmim][OTf]. The peak at $180 \mathrm{ppm}$ is assigned to the carboxylic acid group according to the literature ${ }^{39}$ and further supported by our IR spectroscopy data for the humins formed in [bmim] $\left[\mathrm{HSO}_{4}\right]$. This signal is similar to the chemical shift of the aldehyde in HMF (displaced to lower field than typical values), therefore the IR provides important confirmation of the nature of this functional group. ${ }^{40}$ The resonance at 155 ppm and those present between 110-130 ppm are assigned to the carbon nuclei in the $\alpha, \beta$ positions of the furan ring. The resonances for the ether linkage are observed in the region below $70 \mathrm{ppm}$ along with features for other $\mathrm{sp}^{3}$-like carbon atoms derived from other types of degradation. Compared with humins derived from hydrothermal treatment of sugars in water, the aliphatic region is characterized by multiple peaks suggesting different type of linkers. Typically the $\alpha$ carbon peak at $155 \mathrm{ppm}$ is not observed for humins formed hydrothermally, indicating that structural changes occur to the furan ring in these materials. ${ }^{41}$ Moreover, in contrast to humins derived from thermal or alkali treatments, ${ }^{39,42}$ no signals are observed in the characteristic regions for aldehyde and ketone resonances (above 200 ppm) for the humins formed in the ionic liquid. 
a)

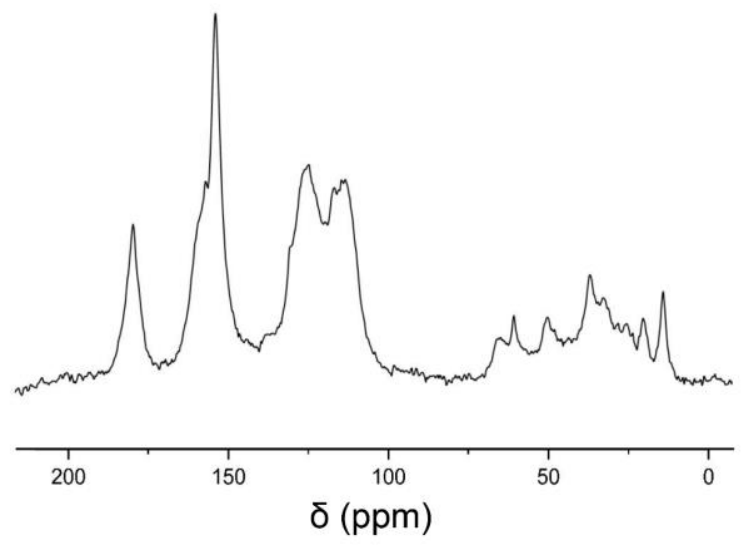

b)

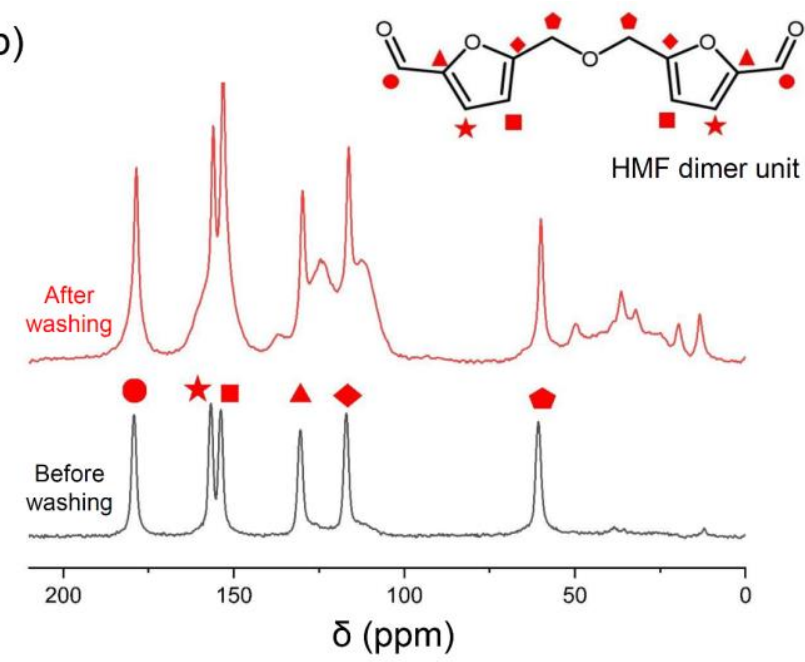

Figure 6. Solid state ${ }^{13} \mathrm{C}\left\{{ }^{1} \mathrm{H}\right\}$ NMR spectra for humins derived from a) [bmim] $\left[\mathrm{HSO}_{4}\right]$ and b) [bmim][OTf] before and after washing with organic solvents (acetone and ethanol).

The spectrum of the humins derived from [bmim][OTf] shows a different pattern, which suggests that the solid is comprised mostly of small oligomers formed from HMF dimers derived from the condensation of the alcohol group. This is in agreement with the mass spectrometry data, which identified a dimer corresponding to a mass of $m / z$ 237. In Figure $6 b,{ }^{13} \mathrm{C}\left\{{ }^{1} \mathrm{H}\right\}$ NMR resonances were assigned to the HMF dimer but no signals were observed for the HMF tetramer in Figure 5 detected by mass spectrometry, possibly due to the low quantity of this molecule compared to the amounts of oligomers formed. In order to analyze the core structure of the solid, it was decided to wash the humins extensively with tetrahydrofuran and ethanol and re-analyze the washed solid. The ${ }^{13} \mathrm{C}\left\{{ }^{1} \mathrm{H}\right\}$ NMR (Figure $6 \mathrm{~b}$ ) and FT-IR (Figure S22) spectra exhibited a background with humin features similar to those for the material formed in [bmim] $\left[\mathrm{HSO}_{4}\right]$, indicating a different composition at the surface (oligomers) compared to the core (more complex polymer matrix). The fact that these oligomers are not observed when humins are formed in [bmim] Br and [bmim] $\left[\mathrm{HSO}_{4}\right]$ suggests that, in the absence of hydrogen bond accepting anions, two different types of degradation arise, which lead to the formation of small, water-insoluble oligomers and polymers derived from ring-opening reactions. Washing the humins formed in [bmim] $\left[\mathrm{HSO}_{4}\right]$ with organic solvents did not lead to any change in the spectrum obtained.

\section{XPS analysis}

In order to gain further insights into the nature of the chemical bonding present in the solids, X-ray photoelectron spectroscopy (XPS) was performed. The main challenge associated with this spectroscopic technique is the tuning of the different parameters (number of components in a peak, background, line shapes) for an accurate fitting of the data. This challenge is greater for carbonaceous materials due to the complexity inherent in the interpretation of carbon $\mathrm{C} 1 \mathrm{~s} \mathrm{X}$-ray photoelectron spectra. Humins produced from [bmim][OTf] and [bmim] $\left[\mathrm{HSO}_{4}\right]$ were analyzed and compared (Figure 7 ). The data from the humins produced in [bmim][OTf] can be fitted well displaying little evidence of C-C peaks, indicating that most of the molecular structure can be approximated to a furanic network with few C-C aliphatic linkages. However, it is important to note that this is mostly indicative of the surface of the humins which, as demonstrated in the solid state NMR analysis, is mostly covered by oligomers. The distribution of functional groups in the HMF tetramer unit shown in Figure 5 matches the pattern obtained 
from the fitting in Figure 7 of the number of carbonyl and acetal groups for the humins prepared in [bmim][OTf]. However, the data for the furanic $\mathrm{C}=\mathrm{C}$ and ether $\mathrm{C}-\mathrm{O}$ linkages indicate that other reactions, which were not detected by IR spectroscopy may exist. This is further confirmed by the presence of a peak (with tailing) that is characteristic for the carbon atoms of carboxylic acids, suggesting that ring opening may also occur, which is further confirmed by the solid state NMR and IR spectra in Figure 6 and Figure S22 (after washing). The fitting obtained for the humins obtained in [bmim] $\left[\mathrm{HSO}_{4}\right]$ shows an increase in the amount of carboxylic acid units present and a smaller number of carbonyl and acetal groups (Table 2), which is in agreement with the IR data (Figure 4). Further C-C bonding was added to the fitting following the evidence from the NMR analysis, which shows signals in the (high-field) aliphatic region.

a)

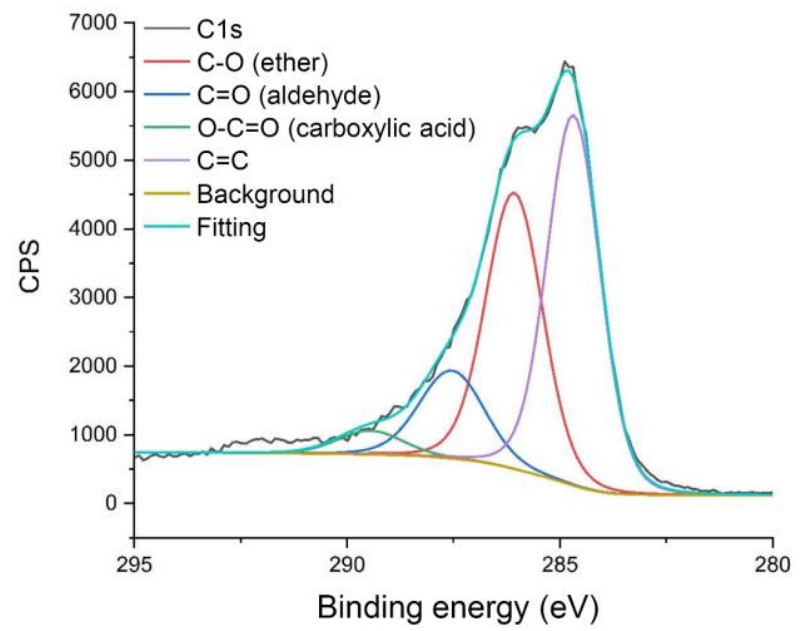

b)

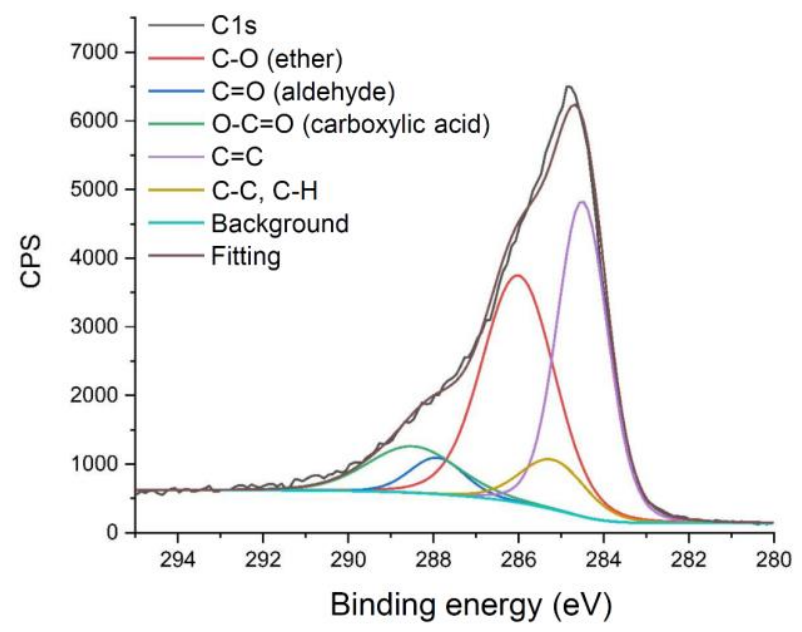

Figure 7. XPS spectra with fitting of humin functional groups for humins produced in a) [bmim][OTf] and b) $[$ bmim $]\left[\mathrm{HSO}_{4}\right]$.

Table 2. Percentage of different functional groups in the humin products derived from the fitting of the C1s XPS signal.

\begin{tabular}{|c|c|c|c|}
\hline Chemical bond & $\begin{array}{l}\text { Humins from } \\
\text { [bmim][OTf] (\%) }\end{array}$ & $\begin{array}{c}\text { Humins from } \\
{\left[\text { bmim] }\left[\mathrm{HSO}_{4}\right](\%)\right.}\end{array}$ & $\begin{array}{c}\text { Isolated tetramer } \\
\text { unit (\%) }\end{array}$ \\
\hline $\mathrm{H}_{2} \mathbf{C}-\mathrm{O}^{\text {ether }}$ & 36.7 & 39.3 & 50.0 \\
\hline $\mathrm{HC}=\mathrm{O}, \mathrm{O}-\mathbf{C}(\mathrm{H})-\mathrm{O}^{\text {acetal }}$ & 13.5 & 4.8 & 16.7 \\
\hline $\mathbf{C}(=\mathrm{O}) \mathrm{OH}$ & 3.6 & 10.0 & - \\
\hline $\mathrm{C}=\mathrm{C}$ & 46.2 & 38.1 & 33.3 \\
\hline Other sp³-like $\mathbf{C}-\mathbf{C}, \mathbf{C}-\mathbf{H}$ & - & 7.8 & - \\
\hline
\end{tabular}

\section{GPC analysis of humins}

The molecular weight of the fraction of humins soluble in dimethyl sulfoxide (DMSO) was analyzed by gel permeation chromatography (GPC) at different time points during the course of the reaction (Figure 8). The humins from the triflate-based ionic liquids exhibited two peaks at low and high molecular weight. While the low molecular weight feature has a sharp peak, its value is not completely reliable since it is below the calibration curve. However, these data are representative for water-insoluble oligomers with low molecular weights. A time course analysis (Figure 8b) of the reaction shows that these low molecular 
weight peaks decrease in intensity in favor of the high molecular weight peak. Indeed, by increasing the reaction time, the molecular weight of the humins increases two-fold with greater polydispersity being observed, as well as a decrease in the peak at lower molecular weight. This indicates that one step in a possible mechanism for humin growth is the agglomeration of oligomers rather than direct addition of HMF to the polymer matrix. This is further confirmed by the IR spectra shown in Figure 5 indicating that the humins are growing with the same repeating units. In contrast, no peaks in the low molecular weight region are observed for humins produced in hydrogensulfate-based ionic liquids. In that case, a mechanism of growth through HMF addition is more plausible compared to the condensation of oligomers.
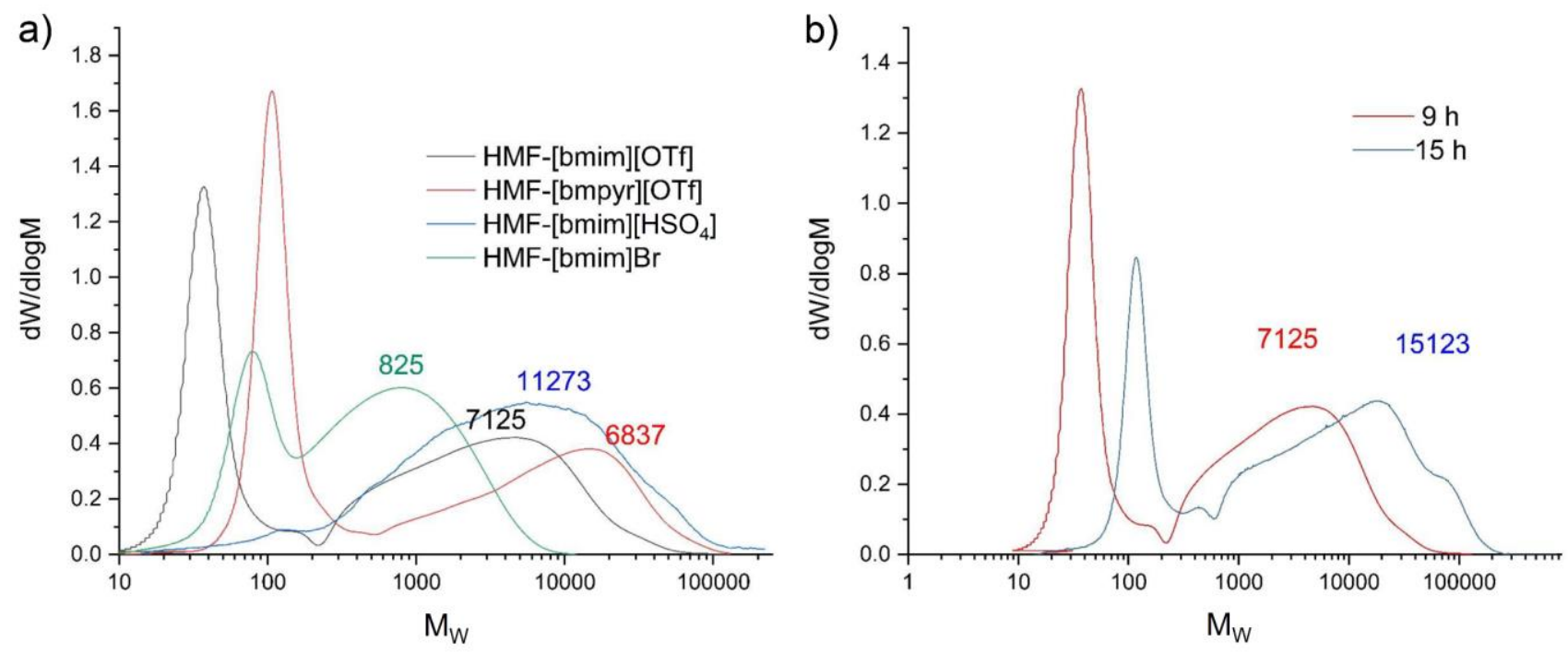

Figure 8. GPC analysis of humins formed in different ionic liquids expressed as average molecular weight. a) Molecular weight distribution of the different humins formed in different ionic liquids and $b$ ) influence of time on the molecular weight of the humins formed in [bmim][OTf].

\section{Morphology of humins}

The morphology of humins was analyzed by scanning electron microscopy (SEM) in order to gain insights regarding their surface architecture. In contrast to previous reports, which reported the formation of humins from HMF in water and water/DMSO mixtures, the humins formed in the different ionic liquids do not all display a spherical morphology. This is likely to be due to a combination of factors, such as the diverse chemical structure of the humins obtained and the differing viscosity of the ionic liquid media. ${ }^{41,43,44}$ In order to allow a comparison with other carbonaceous materials, the morphology of commercial activated carbon was analyzed alongside that of the humins formed in different ionic liquids (Figure 9). The activated carbon surface is shown in Figure 9a and it is evident that the solid is formed by the agglomeration of nanoparticles which gives high porosity and high surface area $\left(948 \mathrm{~m}^{2} \mathrm{~g}^{-1}\right)$. The images of the humins formed in $[\mathrm{bmim}]\left[\mathrm{HSO}_{4}\right]$ partly resemble those of activated carbon, with a hierarchical structure consisting of small particles agglomerated into larger spherical particles - a feature observed in other studies of humins formed from sugars. ${ }^{14}$ However, the humins formed in [bmim]Br display no nanostructured features and the solid is mainly composed of large particles with very low porosity. Since, the IR data indicate that the humins formed by these two ionic liquids have the same structure, other factors (such as viscosity) could be controlling the morphology of the humins and the 
agglomeration of the nanoparticles. The humins formed from the non-coordinating triflate ionic liquid do not display a hierarchical structure and the solid is formed by the agglomeration of large and small particles. Further confirmation of the differing sizes present is obtained from the measurement of the particle size of the humins formed in [bmim][OTf], which indicates an average particle size of $37 \mu \mathrm{m}$ with a broad distribution in the nanometer range (Figure 10). All humins exhibited low surface area $\left(<1 \mathrm{~m}^{2}\right.$ $\mathrm{g}^{-1}$ ), as would be expected in the absence of high temperature treatment. Various different treatments have been reported in the literature to increase the surface area, such as chemical activation with $\mathrm{KOH}$ or pyrolysis. ${ }^{45}$
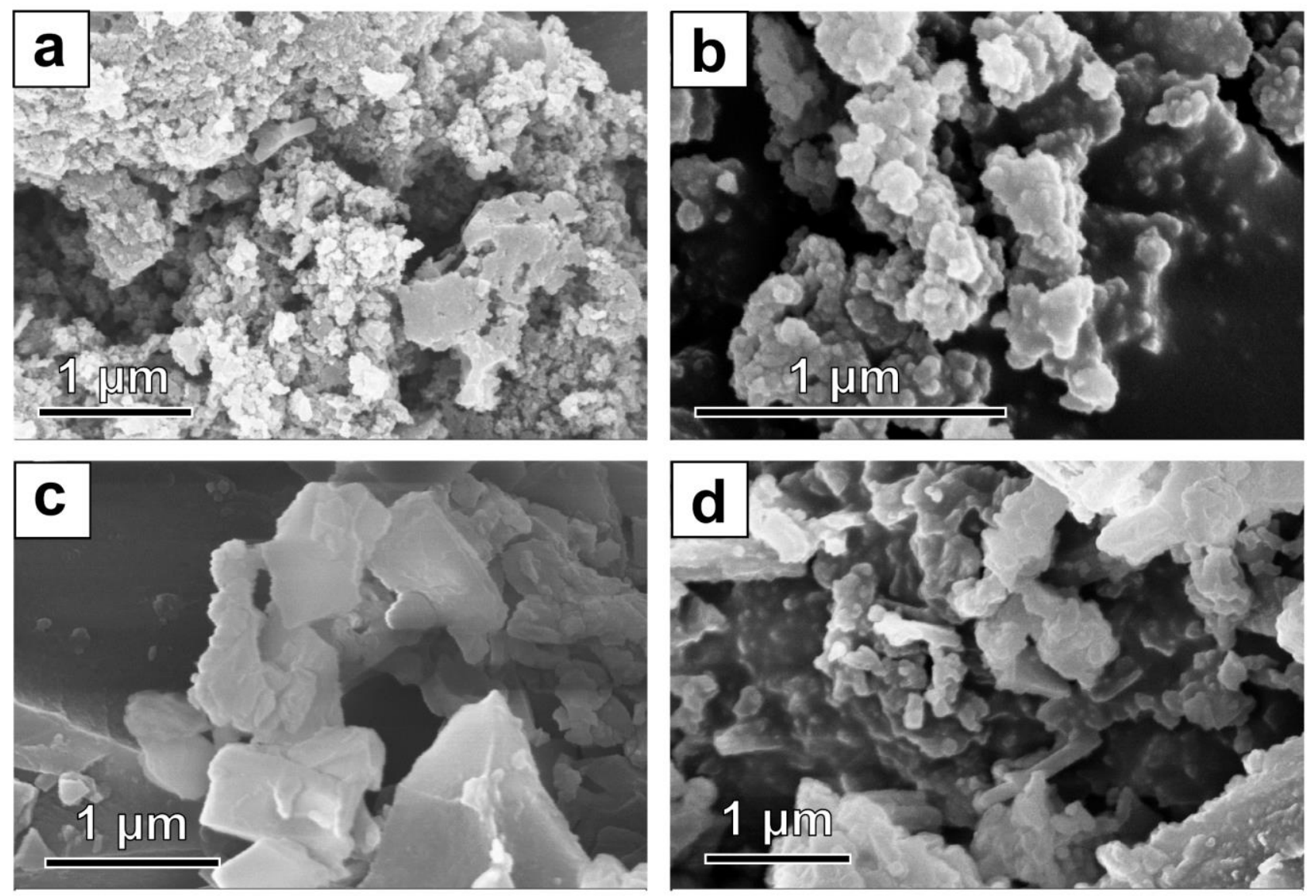

Figure 9. SEM images showing the morphology of a) commercial activated carbon and the humins formed in (b) [bmim] [HSO 4$]$, (c) [bmim] Br, (d) [bmim][OTf]. 


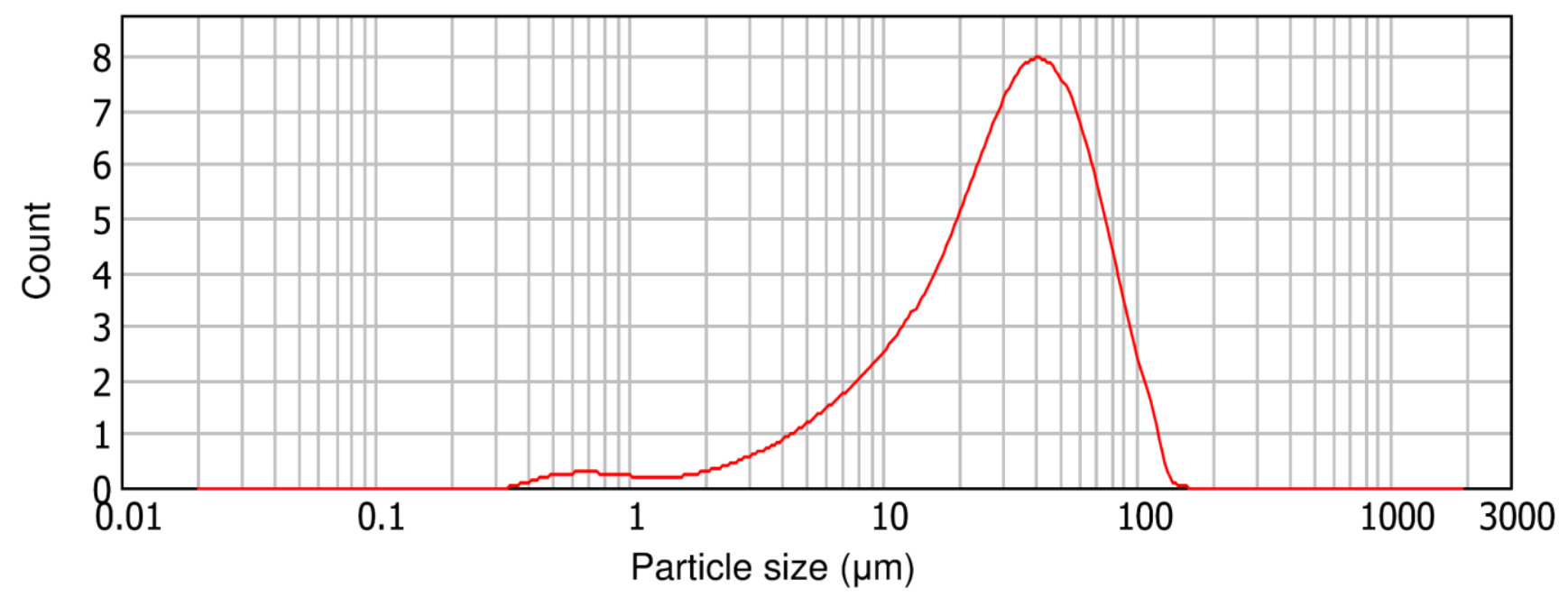

Figure 10. Particle size distribution of humins formed in [bmim][OTf] as determined by laser diffraction particle analysis.

\section{Extraction of metals from water using humins}

Activated carbon is a widely used adsorbent for the removal of organic pollutants and metals from waste water. The mechanism of extraction by activated carbon is physical adsorption, a mass transfer process that leads to the accumulation of adsorbates at the surface via weak, physical forces, such as Van der Waals interactions. The removal efficiency of activated carbon towards a wide range of contaminants has been extensively investigated. ${ }^{46-48}$ In our experiments, the good performance of activated carbon towards lead $\left(\mathrm{Pb}^{2+}\right)$ was confirmed with extraction efficiency values close or equal to $100 \%$, even at very low initial concentrations (Figure 11a). This performance was not matched by any of the humins. In fact, the highest percentage removal $(60 \%)$ was shown by the humins derived from [bmpyr][OTf] at $30 \mathrm{ppm}$ lead loading and this decreased for higher initial concentrations, indicating low absorption capacity. While the extraction of lead by activated carbon is clearly superior, the affinity of the humins towards antimony $\left(\mathrm{Sb}^{3+}\right)$ showed the opposite trend, greatly surpassing that for activated carbon. Almost complete extraction of antimony ions was observed when using the humins formed in [bmim] $\left[\mathrm{HSO}_{4}\right]$, even at high heavy metal ion concentrations (Figure 11b). A plausible rationale for this performance is the selective affinity of the metal species towards the structural and chemical properties of the humins, even if the surface area of the activated carbon is much higher $\left(948 \mathrm{~m}^{2} \mathrm{~g}^{-1}\right.$ for activated carbon, $0.1 \mathrm{~m}^{2}$ $\mathrm{g}^{-1}$ for humins formed in [bmim] [HSO $], 0.25 \mathrm{~m}^{2} \mathrm{~g}^{-1}$ for humins formed in [bmim][OTf]). Accordingly, the greater degree of functionalization of humins compared to that of activated carbon is likely to enhance the observed high adsorption efficiency. Further evidence for this is provided by the XPS data for the antimony adsorbed on the surface of the different carbonaceous materials studied (Figure 12). The XPS analysis for antimony is characterized by two distinct peaks corresponding to $S b 3 \mathrm{~d}_{5 / 2}$ and $\mathrm{Sb}_{3} \mathrm{~d}_{3 / 2}$. However, since the $\mathrm{Sb}_{3} \mathrm{~d}_{5 / 2}$ peak overlaps with the O1s region (from functional groups in the humins) between $528-532 \mathrm{eV}$, the analysis of these features is complex. In contrast, the peak at $\mathrm{Sb}_{3 / 2}$ is not obscured, making its interpretation more straightforward. From the analysis of the $\mathrm{Sb3d}_{3 / 2}$ peak in the region $541-538 \mathrm{eV}$, it can be deduced that the binding energy of the peak decreases in the order: activated carbon $(540.08 \mathrm{eV})$, humins from [bmim][OTf] $(539.58 \mathrm{eV})$ and humins from [bmim] $\left[\mathrm{HSO}_{4}\right]$ $(539.10 \mathrm{eV})$, indicating that the humins are interacting more strongly with the valence electrons of the antimony in the humins with respect to its situation in activated carbon. This suggests that the chemical 
oxygen-based functionality characteristic of humins leads to stronger binding to antimony than is the case for activated carbon. The observed trend in binding energy values reflects the propensity of these carbon-based materials to adsorb antimony ions. This lends support to the hypothesis that it is the chemical functionality of the humins that leads to the enhanced adsorption of antimony ions. In particular, the carboxylate functionality present offers a stronger interaction with antimony compared to (non-chemically treated and hence unfunctionalized) activated carbon. ${ }^{49,50}$ Various studies have reported the removal of antimony from water and these have highlighted the importance of the presence of carboxylic groups. ${ }^{51}$ In particular, Castaldi and co-workers showed that antimony can be removed by chelation with citric and oxalic acids before addition of ethylenediaminetetraacetic acid (EDTA). ${ }^{52}$

An analysis of the fitting with the Freundlich isotherm suggests that activated carbon has an adsorption capacity of $214 \mathrm{mg} / \mathrm{g}$ compared to around $341 \mathrm{mg} / \mathrm{g}$ for [bmim][OTf]. For [bmim][HSO 4 ], values over $500 \mathrm{mg} / \mathrm{g}$ can be achieved, further indicating the high adsorption capacity of this solid material due to its high degree of functionality.

It is known that a significant amount of antimony enters the environment every year in different forms on account of the widespread use of antimony compounds in agriculture and industry. Both antimony and antimonide are toxic and exposure to sources of antimony in water presents a significant risk to humans. Currently the removal of antimony faces various challenges and the different methodologies employed suffer from distinct drawbacks. For example, activated carbon needs to be doped with iron to enhance the extraction process. Coagulation/flocculation requires additives which contribute to the generation of toxic waste while other approaches, such as membrane filtration or electrodeposition, are expensive. ${ }^{53}$ The investigation of humins for the removal of antimony is thus worthy of further attention.
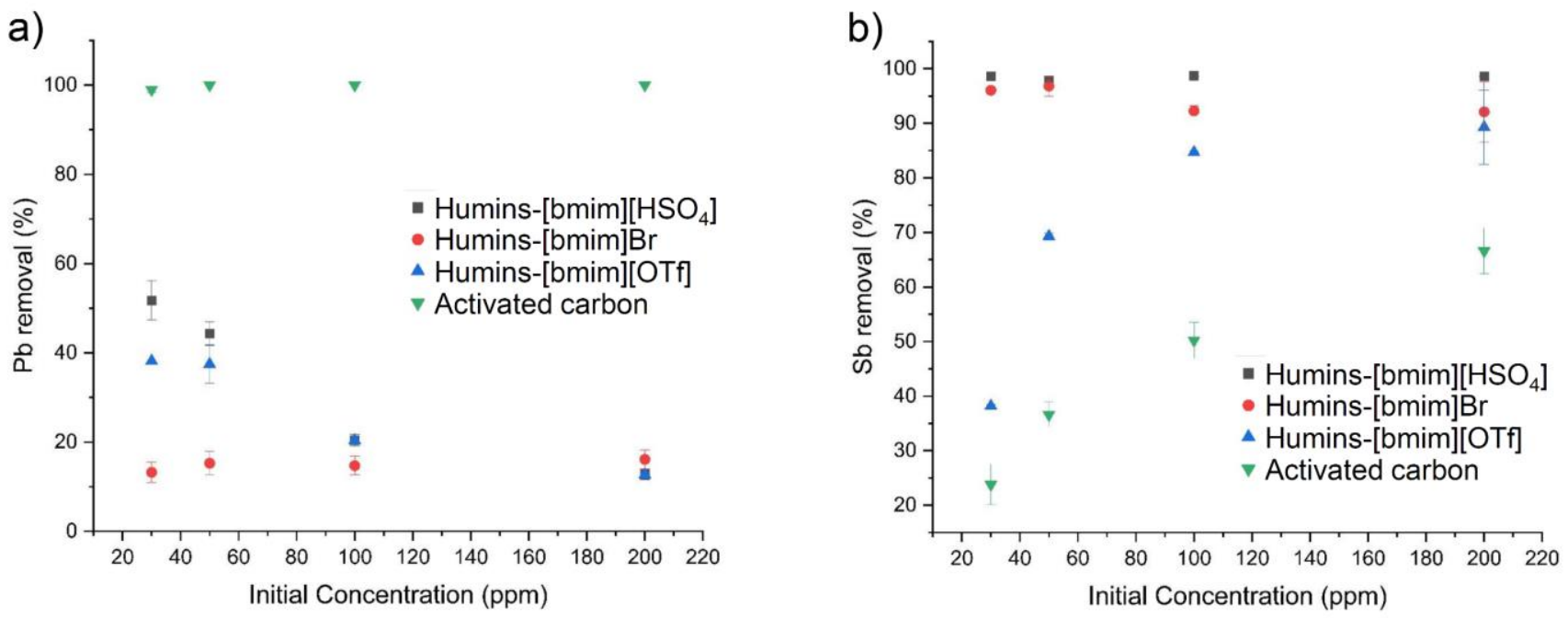

Figure 11. Extraction of a) $\mathrm{Pb}^{2+}$ ions and b) $\mathrm{Sb}^{3+}$ ions using humins produced in different ionic liquids as well as activated carbon. Carbonaceous solid loading is $1 \%$ by mass with respect to the solvent. 


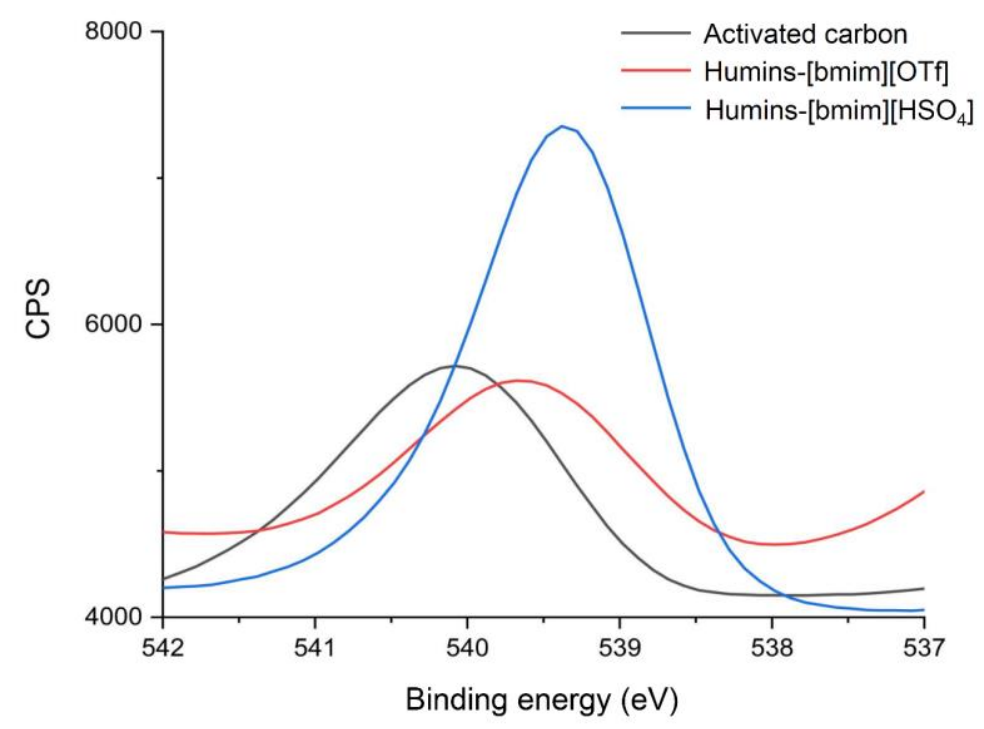

Figure 12. XPS data for the $\mathrm{Sb}_{3} \mathrm{~d}_{3 / 2}$ signal of trivalent antimony adsorbed on the surface of the humins produced in this work and activated carbon.

\section{CONCLUSIONS}

These investigations have allowed the stability of HMF towards the formation of humins to be analyzed in various ionic liquids, illustrating the impact of the reaction medium on the resulting carbonaceous materials. Spectroscopic techniques, such as infrared spectroscopy, solid state ${ }^{13} \mathrm{C}\left\{{ }^{1} \mathrm{H}\right\} \mathrm{NMR}$ spectroscopy and XPS were used to analyze the main functional groups in the solid. For the humins formed in non-coordinating ionic liquids (with triflate anions), a mechanism of degradation was proposed based on a possible tetramer unit. In contrast, the humins formed in ionic liquids with anions capable of hydrogen bonding (bromide and hydrogensulfate) share the same chemical structure, characterized by a greater proportion of carboxylic acid groups, but display different morphologies. Humins formed in [bmim] $\left[\mathrm{HSO}_{4}\right]$ exhibit a hierarchical structure (SEM) which consists of nanoparticles agglomerated into larger spherical particles. In addition, the formation of humins in ionic liquids requires milder, more manageable conditions $\left(160{ }^{\circ} \mathrm{C}\right.$ and $1 \mathrm{~atm}$ ) compared to their formation in water (typically $180{ }^{\circ} \mathrm{C}$ and $20 \mathrm{~atm}),{ }^{13-15}$ reducing safety concerns. The improved understanding of the differences in structure and functionality gained through this study was applied to the important and topical application of heavy metal ion removal from water. It was found that the more highly functionalized humins were most effective. This higher degree of functionality is suggested to be the reason for the markedly superior performance observed in the removal of antimony ions from water compared to widely-used activated carbon. This finding is important as the removal of this particular wastewater contaminant has been inefficient using conventional or chemically-treated activated carbon. ${ }^{25,26}$ Furthermore, the mild conditions used to form this functional material can lead to energy savings since no activation procedure using steam or chemical treatment is required. This potential application is significant both in terms of the increasing issues of antimony pollution and the likely production of humins as side products in the biorefineries of the future.

\section{ASSOCIATED CONTENT}

The Supporting Information is available free of charge at:

Synthetic procedures and characterization with selected spectra and analytical data (PDF) 


\section{AUTHOR INFORMATION \\ Corresponding Authors}

Amir Al Ghatta - Department of Chemical Engineering, Imperial College London, South Kensington Campus, Imperial College Road, London SW7 2AZ, United Kingdom and Department of Chemistry, Imperial College London, Molecular Sciences Research Hub, White City Campus, 82 Wood Lane, London W12 0BZ, United Kingdom; orcid.org/0000-0002-0599-1494.

Email: a.al-ghatta16@imperial.ac.uk.

James D. E. T. Wilton-Ely - Department of Chemistry, Imperial College London, Molecular Sciences Research Hub, White City Campus, 82 Wood Lane, London W12 0BZ, United Kingdom; orcid.org/00000002-5192-3038.

Email: j.wilton-ely@imperial.ac.uk.

Jason P. Hallett - Department of Chemical Engineering, Imperial College London, South Kensington Campus, Imperial College Road, London SW7 2AZ, United Kingdom; orcid.org/0000-0003-3431-2371. Email: j.hallett@imperial.ac.uk.

\section{Authors}

Xinyi Zhou - Department of Chemical Engineering, Imperial College London, South Kensington Campus, Imperial College Road, London SW7 2AZ, United Kingdom and Department of Chemistry, Imperial College London, Molecular Sciences Research Hub, White City Campus, 82 Wood Lane, London W12 0BZ, United Kingdom.

Giulia Casarano - Department of Chemistry, Imperial College London, Molecular Sciences Research Hub, White City Campus, 82 Wood Lane, London W12 0BZ, United Kingdom.

\section{Notes}

The authors declare no competing financial interest.

\section{ACKNOWLEDGEMENTS}

Imperial College London is thanked for the award of a President's PhD Scholarship (to A.A.G.) and the EPSRC are gratefully acknowledged for a Doctoral Prize Fellowship (to A.A.G.). Prof. R. Law is acknowledged for the measurement of solid state NMR spectra. We thank Prof. M. Titirici for helpful discussions. We thank Dr G. Kerherve for measurements and technical assistance in the XPS analysis. Dr C. Clarke is acknowledged for support in the interpretation of the XPS spectra.

\section{REFERENCES}

(1) Ragauskas, A. J.; Williams, C. K.; Davison, B. H.; Britovsek, G.; Cairney, J.; Eckert, C. A.; Frederick Jr., W. J.; Hallett, J. P.; Leak, D. J.; Liotta, C. L.; et al. The Path Forward for Biofuels and Biomaterials. Science 2006, 311 (5760), 484-489. https://doi.org/10.1126/science.1114736.

(2) Dashtban, M.; Gilbert, A.; Fatehi, P. Recent Advancements in the Production of Hydroxymethylfurfural. RSC Adv. 2014, 4 (4), 2037-2050. https://doi.org/10.1039/c3ra45396k.

(3) Zakrzewska, M. E.; Bogel-łukasik, E.; Bogel-łukasik, R. Ionic Liquid-Mediated Formation of 5- 
Hydroxymethylfurfural s A Promising Biomass-Derived Building Block. Chem. Rev. 2011, 111 (2), 397-417. https://doi.org/https://doi.org/10.1021/cr100171a.

(4) Al Ghatta, A.; Wilton-Ely, J. D. E. T.; Hallett, J. P. Strategies for the Separation of the Furanic Compounds HMF, DFF, FFCA, and FDCA from lonic Liquids. ACS Sustainable Chem. Eng. 2019, 7 (19), 16483-16492. https://doi.org/https://doi.org/10.1021/acssuschemeng.9b03613.

(5) Galkin, K. I.; Krivodaeva, E. A.; Romashov, L. V.; Zalesskiy, S. S.; Kachala, V. V.; Burykina, J. V.; Ananikov, V. P. Critical Influence of 5-Hydroxymethylfurfural Aging and Decomposition on the Utility of Biomass Conversion in Organic Synthesis. Angew. Chem. Int. Ed. 2016, 55 (29), 83388342. https://doi.org/10.1002/anie.201602883.

(6) Galkin, K. I.; Ananikov, V. P. Towards Improved Biorefinery Technologies : 5-Methylfurfural as a Versatile C6 Platform for Biofuels Development. ChemSusChem 2019, 12 (1), 185-189. https://doi.org/10.1002/cssc.201802126.

(7) Al Ghatta, A.; Wilton-Ely, J. D. E. T.; Hallett, J. P. Efficient Formation of 2,5-Diformylfuran in lonic Liquids at High Substrate Loadings and Low Oxygen Pressure with Separation through Sublimation. ACS Sustainable Chem. Eng. 2020, 8 (6), 2462-2471. https://doi.org/10.1021/acssuschemeng.9b06691.

(8) Stahlberg, T.; Eyjolfsdottir, E.; Gorbanev, Y. Y.; Sdaba, I.; Riisager, A. Aerobic Oxidation of 5(Hydroxymethyl)Furfural in Ionic Liquids with Solid Ruthenium Hydroxide Catalysts. Catal. Letters 2012, 142 (9), 1089-1097. https://doi.org/10.1007/s10562-012-0858-5.

(9) Yan, D.; Xin, J.; Zhao, Q.; Gao, K.; Lu, X.; Wang, G.; Zhang, S. Fe-Zr-O Catalyzed Base-Free Aerobic Oxidation of 5-HMF to 2,5-FDCA as a Bio-Based Polyester Monomer. Catal. Sci. Technol. 2018, 8 (1), 164-175. https://doi.org/10.1039/c7cy01704a.

(10) Yan, D.; Xin, J.; Shi, C.; Lu, X.; Ni, L.; Wang, G.; Zhang, S. Base-Free Conversion of 5Hydroxymethylfurfural to 2,5-Furandicarboxylic Acid in Ionic Liquids. Chem. Eng. J. 2017, 323 (1), 473-482. https://doi.org/10.1016/j.cej.2017.04.021.

(11) Chen, R.; Xin, J.; Yan, D.; Dong, H.; Lu, X.; Zhang, S. Highly Efficient Oxidation of 5Hydroxymethylfurfural to 2,5-Furandicarboxylic Acid with Heteropoly Acids. ChemSusChem 2019, 12 (12), 2715-2724. https://doi.org/10.1002/cssc.201900651.

(12) 12 Principles of Green Chemistry - American Chemical Society https://www.acs.org/content/acs/en/greenchemistry/principles/12-principles-of-greenchemistry.html (accessed Dec 3, 2020).

(13) Hoang, T. M. C.; van Eck, E. R. H.; Bula, W. P.; Gardeniers, J. G. E.; Leffertsa, L.; Seshana, K. Humin Based By-Products from Biomass Processing as a Potential Carbonaceous Source for Synthesis Gas Production. Green Chem. 2015, $17 \quad$ (2), 959-972. https://doi.org/10.1039/C4GC01324G.

(14) Patil, S. K. R.; Lund, C. R. F. Formation and Growth of Humins via Aldol Addition and Condensation during Acid-Catalyzed Conversion of 5-Hydroxymethylfurfural. Energy Fuels 2011, 25 (10), 4745-4755. https://doi.org/10.1021/ef2010157.

(15) Cheng, Z.; Vlachos, D. G.; Everhart, J. L.; Tsilomelekis, G.; Nikolakis, V.; Saha, B. Structural Analysis of Humins Formed in the Brønsted Acid Catalyzed Dehydration of Fructose. Green Chem. 2018, 20 (5), 997-1006. https://doi.org/10.1039/C7GC03054A.

Al Ghatta, A.; Wilton-Ely, J. D. E. T.; Hallett, J. P. Rapid, High-Yield Fructose Dehydration to 5- 
Hydroxymethylfurfural in Mixtures of Water and the Noncoordinating lonic Liquid [Bmim][OTf]. ChemSusChem 2019, 12

(19), 4452-4460. https://doi.org/https://doi.org/10.1002/cssc.201901529.

(17) Li, Y. N.; Wang, J. Q.; He, L. N.; Yang, Z. Z.; Liu, A. H.; Yu, B.; Luan, C. R. Experimental and Theoretical Studies on Imidazolium Ionic Liquid-Promoted Conversion of Fructose to 5Hydroxymethylfurfural. Green Chem. 2012, $14 \quad$ (10), 2752-2758. https://doi.org/10.1039/c2gc35845j.

(18) Zhao, H.; Holladay, J. E.; Brown, H.; Zhang, Z. C. Metal Chlorides in lonic Liquid Solvents Convert Sugars to 5-Hydroxymethylfurfural. Science 2007, 316 (5831), 1597-1600. https://doi.org/10.1126/science.1141199.

(19) Wei, Z.; Liu, Y.; Thushara, D.; Ren, Q. Entrainer-Intensified Vacuum Reactive Distillation Process for the Separation of 5-Hydroxylmethylfurfural from the Dehydration of Carbohydrates Catalyzed by a Metal Salt-Ionic Liquid. Green Chem. 2012, 14 (4), 1220-1226. https://doi.org/10.1039/c2gc16671b.

(20) Zhang, Z.; Liu, W.; Xie, H.; Zhao, Z. K. An Unexpected Reaction between 5-Hydroxymethylfurfural and Imidazolium-Based lonic Liquids at High Temperatures. Molecules 2011, 16 (10), 84638474. https://doi.org/10.3390/molecules16108463.

(21) Ma, Z.; Yu, J.; Dai, S. Preparation of Inorganic Materials Using lonic Liquids. Adv. Mater. 2010, 22 (2), 261-285. https://doi.org/10.1002/adma.200900603.

(22) Paraknowitsch, J. P.; Zhang, J.; Su, D.; Thomas, A.; Antonietti, M. lonic Liquids as Precursors for Nitrogen-Doped Graphitic Carbon. Adv. Mater. 2010, 22 (1), 87-92. https://doi.org/10.1002/adma.200900965.

(23) Xie, Z. L.; White, R. J.; Weber, J.; Taubert, A.; Titirici, M. M. Hierarchical Porous Carbonaceous Materials via lonothermal Carbonization of Carbohydrates. J. Mater. Chem. 2011, 21 (20), 74347442. https://doi.org/10.1039/c1jm00013f.

(24) Hu, B.; Wang, K.; Wu, L.; Yu, S.-H.; Antonietti, M.; Titirici, M.-M. Engineering Carbon Materials from the Hydrothermal Carbonization Process of Biomass. Adv. Mater. 2010, 22 (7), 813-828. https://doi.org/10.1002/adma.200902812.

(25) Yu, T.; Wang, X.; Li, C. Removal of Antimony by FeCl3-Modified Granular-Activated Carbon in Aqueous Solution. J. Environ. Eng. 2014, 140 (9). https://doi.org/10.1061/(ASCE)EE.19437870.0000736.

(26) Lin, B. L.; Hu, J. W.; Duan, S. M.; Huang, X. F.; Jia, M.; Wang, Y.; Liu, C.; Luo, J. A Study on Adsorption Kinetics of Antimony onto Coconut Shell Activated Carbon. Adv. Mater. Res. 2013, 788, 471-475.

(27) XPS Interpretation of Carbon https://xpssimplified.com/elements/carbon.php (accessed Dec 3, 2020).

(28) Spange, S.; Lungwitz, R.; Schade, A. Correlation of Molecular Structure and Polarity of lonic Liquids. J. Mol. Liq. 2014, 192, 137-143. https://doi.org/10.1016/j.molliq.2013.06.016.

(29) Brandt, A.; Ray, M. J.; To, T. Q.; Leak, D. J.; Murphy, R. J.; Welton, T. Green Chemistry lonic Liquid Pretreatment of Lignocellulosic Biomass with lonic Liquid-Water Mixtures. Green Chem. 2011, 13 (9), 2489-2499. https://doi.org/10.1039/c1gc15374a. 
(30) Alarcón-Espósito, J.; Contreras, R.; Tapia, R. A.; Campodónico, P. R. Gutmann's Donor Numbers Correctly Assess the Effect of the Solvent on the Kinetics of $S_{N}$ Ar Reactions in lonic Liquids. Chem. Eur. J. 2016, 22 (37), 13347-13351. https://doi.org/10.1002/chem.201602237.

(31) Wang, H.; Liu, S.; Zhao, Y.; Zhang, H.; Wang, J. Molecular Origin for the Difficulty in Separation of 5-Hydroxymethylfurfural from Imidazolium Based lonic Liquids. ACS Sustainable Chem. Eng. 2016, 4 (12), 6712-6721. https://doi.org/10.1021/acssuschemeng.6b01652.

(32) Gajula, S.; Inthumathi, K.; Arumugam, S. R.; Srinivasan, K. Strategic Designing on Selection of Solvent Systems for Conversion of Biomass Sugars to Furan Derivatives and Their Separation. ACS Sustainable Chem. Eng. 2017, 5 (6), 5373-5381. https://doi.org/10.1021/acssuschemeng.7b00681.

(33) Van Zandvoort, I.; Wang, Y.; Rasrendra, C. B.; van Eck, E. R. H.; Bruijnincx, P. C. A.; Heeres, H. J.; Weckhuysen, B. M. Formation, Molecular Structure, and Morphology of Humins in Biomass Conversion: Influence of Feedstock and Processing Conditions. ChemSusChem 2013, 6 (9), 1745-1758. https://doi.org/10.1002/cssc.201300332.

(34) Sumerskii, I. V; Krutov, S. M.; Ya Zarubin, M. Humin-Like Substances Formed under the Conditions of Industrial Hydrolysis of Wood. Russ. J. Appl. Chem. 2010, 83 (2), 321-328. https://doi.org/10.1134/S1070427210020266.

(35) Shen, H.; Shan, H.; Liu, L. Evolution Process and Controlled Synthesis of Humins with 5Hydroxymethylfurfural (HMF) as Model Molecule. ChemSusChem 2020, 13 (3), 513-519. https://doi.org/10.1002/cssc.201902799.

(36) Xu, Z.; Yang, Y.; Yan, P.; Xia, Z.; Liu, X.; Zhang, Z. C. Mechanistic Understanding of Humin Formation in the Conversion of Glucose and Fructose to 5-Hydroxymethylfurfural in [BMIM]Cl Ionic Liquid. RSC Adv. 2020, 10, 34732-34737. https://doi.org/10.1039/d0ra05641c.

(37) Xie, Z. L.; Huang, X.; Titirici, M. M.; Taubert, A. Mesoporous Graphite Nanoflakes via lonothermal Carbonization of Fructose and Their Use in Dye Removal. RSC Adv. 2014, 4 (70), 37423-37430. https://doi.org/10.1039/c4ra05146g.

(38) Eminov, S.; Wilton-Ely, J. D. E. T.; Hallett, J. P. Highly Selective and Near-Quantitative Conversion of Fructose to 5-Hydroxymethylfurfural Using Mildly Acidic Ionic Liquids. ACS Sustainable Chem. Eng. 2014, 2 (4), 978-981. https://doi.org/10.1021/sc400553q.

(39) Van Zandvoort, I.; Koers, E. J.; Weingarth, M.; Bruijnincx, P. C. A.; Baldus, M.; Weckhuysen, B. M. Structural Characterization of 13C-Enriched Humins and Alkali-Treated 13C Humins by 2D Solid-State NMR. Green Chem. 2015, 17 (8), 4383-4392. https://doi.org/10.1039/c5gc00327j.

(40) Zhang, M.; Yang, H.; Liu, Y.; Sun, X.; Zhang, D.; Xue, D. First Identification of Primary Nanoparticles in the Aggregation of HMF. Nanoscale Res. Lett. 2012, 7 (1), 38. https://doi.org/10.1186/1556-276X-7-38.

(41) Falco, C.; Baccile, N.; Titirici, M. M. Morphological and Structural Differences between Glucose, Cellulose and Lignocellulosic Biomass Derived Hydrothermal Carbons. Green Chem. 2011, 13 (11), 3273-3281. https://doi.org/10.1039/c1gc15742f.

(42) Van Zandvoort, I.; Van Eck, E. R. H.; De Peinder, P.; Heeres, H. J.; Bruijnincx, P. C. A.; Weckhuysen, B. M. Full, Reactive Solubilization of Humin Byproducts by Alkaline Treatment and Characterization of the Alkali-Treated Humins Formed. ACS Sustainable Chem. Eng. 2015, 3 (3), 533-543. https://doi.org/10.1021/sc500772w. 
(43) Tsilomelekis, G.; Josephson, T. R.; Nikolakis, V.; Caratzoulas, S. Origin of 5Hydroxymethylfurfural Stability in Water/Dimethyl Sulfoxide Mixtures. ChemSusChem 2014, 7 (1), 117-126. https://doi.org/10.1002/cssc.201300786.

(44) Tsilomelekis, G.; Orella, M. J.; Lin, Z.; Cheng, Z.; Zheng, W.; Nikolakis, V.; Vlachos, D. G. Molecular Structure, Morphology and Growth Mechanisms and Rates of 5-Hydroxymethyl Furfural (HMF) Derived Humins. Green Chem. 2016, 18 (7), 1983-1993. https://doi.org/10.1039/c5gc01938a.

(45) Wang, J.; Kaskel, S. KOH Activation of Carbon-Based Materials for Energy Storage J. Mater. Chem. 2012, 22, 23710-23725. https://doi.org/10.1039/c2jm34066f.

(46) Taiwo, A. F.; Chinyere, N. J. Sorption Characteristics for Multiple Adsorption of Heavy Metal lons Using Activated Carbon from Nigerian Bamboo. J. Mater. Sci. Chem. Eng. 2016, 4 (4), 39-48. https://doi.org/10.4236/msce.2016.44005.

(47) Bohli, T.; Ouederni, A.; Villaescusa, I. Simultaneous Adsorption Behavior of Heavy Metals onto Microporous Olive Stones Activated Carbon: Analysis of Metal Interactions. Euro-Mediterranean J. Environ. Integr. 2017, 2 (1), 1-15. https://doi.org/10.1007/s41207-017-0030-0.

(48) Karnib, M.; Kabbani, A.; Holail, H.; Olama, Z. Heavy Metals Removal Using Activated Carbon, Silica and Silica Activated Carbon Composite. In Energy Procedia; Elsevier Ltd, 2014; Vol. 50, pp 113-120. https://doi.org/10.1016/j.egypro.2014.06.014.

(49) Sato, S.; Yoshihara, K.; Moriyama, K.; Machida, M.; Tatsumoto, H. Influence of Activated Carbon Surface Acidity on Adsorption of Heavy Metal lons and Aromatics from Aqueous Solution. Appl. Surf. Sci. 2007, 253 (20), 8554-8559. https://doi.org/10.1016/j.apsusc.2007.04.025.

(50) Yin, C. Y.; Aroua, M. K.; Daud, W. M. A. W. Review of Modifications of Activated Carbon for Enhancing Contaminant Uptakes from Aqueous Solutions. Sep. Purif. Technol. 2007, 52 (3), 403-415. https://doi.org/10.1016/j.seppur.2006.06.009.

(51) Mudhoo, A.; Ramasamy, D. L.; Bhatnagar, A.; Usman, M.; Sillanpää, M. An Analysis of the Versatility and Effectiveness of Composts for Sequestering Heavy Metal lons, Dyes and Xenobiotics from Soils and Aqueous Milieus. Ecotoxicol. Environ. Saf. 2020, 197, 110587. https://doi.org/10.1016/j.ecoenv.2020.110587.

(52) Diquattro, S.; Garau, G.; Lauro, G. P.; Silvetti, M.; Deiana, S.; Castaldi, P. Municipal Solid Waste Compost as a Novel Sorbent for Antimony(V): Adsorption and Release Trials at Acidic PH. Environ. Sci. Pollut. Res. 2018, 25 (6), 5603-5615. https://doi.org/10.1007/s11356-017-0933-y.

(53) Li, J.; Zheng, B. H.; He, Y.; Zhou, Y.; Chen, X.; Ruan, S.; Yang, Y.; Dai, C.; Tang, L. Antimony Contamination, Consequences and Removal Techniques: A Review. Ecotoxicol. Environ. Saf. 2018, 156, 125-134. https://doi.org/10.1016/j.ecoenv.2018.03.024. 
For ToC use:

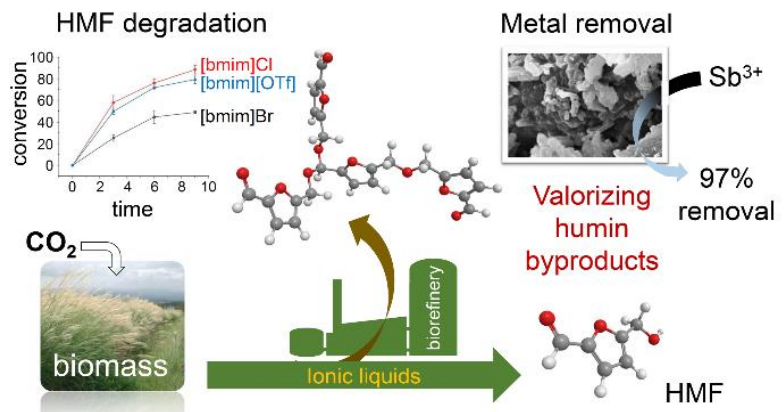

The ionic liquid medium dictates the structure of the humins formed from HMF, leading to their use in antimony removal. 


\section{Supporting information}

Characterization and valorization of humins produced by HMF degradation in ionic liquids: A valuable carbonaceous material for antimony removal

Amir Al Ghatta, ${ }^{\star}$ Xinyi Zhou, Giulia Casarano, James D. E. T. Wilton-Ely, ${ }^{*}$ Jason P. Hallett ${ }^{\star}$

Department of Chemical Engineering, Imperial College, South Kensington Campus, London SW7 $2 A Z$.

Department of Chemistry, Imperial College, Molecular Sciences Research Hub, White City Campus, London W12 OBZ.

\section{Contents}

S1 General considerations

page $\mathrm{S} 2$

S2 Synthesis of ionic liquids with halide anions

page $\mathrm{S} 2$

S2.1 Synthesis of [bmim] Cl

page $\mathrm{S} 2$

S2.2 Synthesis of [bmim] $\mathrm{Br}$

page S5

S2.3 Synthesis of [bmpyr]Cl

page $S 7$

S3 Synthesis of ionic liquids with triflate anions

page 59

S3.1 Synthesis of [bmim][OTf]

page $\$ 9$

S3.2 Synthesis of [bmpyr][OTf]

page $S 11$

S4 Mass spectrometry analysis of oligomers

page $\mathrm{S} 13$

S5 Scanning Electron Microscopy (SEM) images of the materials

page $S 14$

S6 X-ray Photoelectron Spectroscopy (XPS)

page $S 18$

S7 Fourier Transform Infrared Spectroscopy (FT-IR)

page $S 19$

S8 References

page S22

Number of figures: 24

Number of tables: 5 


\section{S1 General considerations}

1-Methylimidazole, 1-chlorobutane, 1-bromobutane, $\mathrm{N}$-methylpyrrolidine, lithium triflate, calcium hydride and phosphorous pentoxide were purchased from Merck. Acetonitrile, ethyl acetate and dichloromethane were procured from VWR. 1-Methylimidazole was distilled under reduced pressure over $\mathrm{CaH}_{2}$. 1-Chlorobutane was distilled at atmospheric pressure under $\mathrm{P}_{2} \mathrm{O}_{5}$. All reagents were used as received unless stated otherwise. Electrospray mass spectra were obtained using a Synapt G2 instrument (in positive mode). Nuclear magnetic resonance (NMR) spectroscopy was performed using a Bruker $400 \mathrm{MHz}$ spectrometer using deuterated DMSO as solvent. All couplings are reported in Hertz. SEM images were obtained using a JSM-6400 scanning electron microscope.

\section{S2 Synthesis of ionic liquids with halide anions}

\section{S2.1 Synthesis of [bmim] Cl}

The ionic liquid [bmim] $\mathrm{Cl}$ was synthesized on a large scale $(350 \mathrm{~g})$. Manipulation of all chemicals was performed by cannulation under inert conditions. 1-methylimidazole $(160 \mathrm{~mL}$, $2 \mathrm{~mol}$ ) was transferred to a three neck flask equipped with an air condenser followed by the addition of acetonitrile $(100 \mathrm{~mL})$. The mixture was heated at $60{ }^{\circ} \mathrm{C}$ under stirring and 1chlorobutane $(230 \mathrm{~mL}, 2.2 \mathrm{~mol}, 1.1 \mathrm{eq})$ was then added dropwise using a dropping funnel. The reaction mixture was heated for 3 days at $70{ }^{\circ} \mathrm{C}$. Complete consumption of 1methylimidazole was confirmed by ${ }^{1} \mathrm{H}$ NMR spectroscopy. [bmim] $\mathrm{Cl}$ was precipitated by gradual addition of ethyl acetate at $3^{\circ} \mathrm{C}$. The resultant white crystals were washed under inert conditions with portions of ethyl acetate ( $2 \mathrm{~L}$ in total), dried under vacuum and stored in a glovebox.

[bmim]Cl: ${ }^{1} \mathrm{H}$ NMR (DMSO-d $\left.\mathrm{d}_{6}\right): \delta 9.62\left(\mathrm{~s}, 1 \mathrm{H}, \mathrm{N}_{2} \mathrm{CH}\right), 7.92,7.84(\mathrm{~m} \times 2,2 \times 1 \mathrm{H}, \mathrm{NCH}), 4.20$ (t, $\left.2 \mathrm{H}, \mathrm{N}-\underline{\mathrm{H}}_{2}-\mathrm{CH}_{2}, \mathrm{~J}_{\mathrm{HH}}=7.2 \mathrm{~Hz}\right), 3.88\left(\mathrm{~s}, 3 \mathrm{H}, \mathrm{N}-\mathrm{CH}_{3}\right), 1.77,1.24\left(\mathrm{~m} \times 2,2 \times 2 \mathrm{H}, \mathrm{N}-\mathrm{CH}_{2}-\underline{\mathrm{C}}_{2}-\right.$ $\left.\mathrm{C}_{2}-\mathrm{CH}_{3}\right), 0.87$ (t, 3H, $\left.-\mathrm{CH}_{2}-\underline{\mathrm{C}}_{3}, \mathrm{~J}_{\mathrm{HH}}=7.3 \mathrm{~Hz}\right) \mathrm{ppm} .{ }^{13} \mathrm{C}\left\{{ }^{1} \mathrm{H}\right\} \mathrm{NMR}\left(\mathrm{DMSO}-\mathrm{d}_{6}\right): \delta 137.2\left(\mathrm{~N}_{2}-\right.$

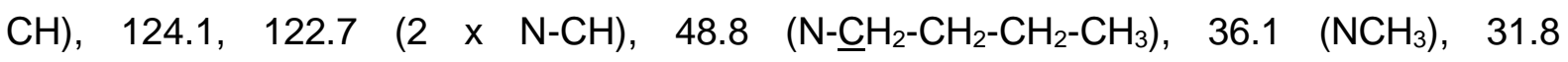
$\left(\mathrm{NCH}_{2} \underline{\mathrm{C}} \mathrm{H}_{2} \mathrm{CH}_{2} \mathrm{CH}_{3}\right), 19.2\left(\mathrm{NCH}_{2}-\mathrm{CH}_{2}-\mathrm{CH}_{2}-\mathrm{CH}_{3}\right), 13.7\left(\mathrm{~N}-\left(\mathrm{CH}_{2}\right)_{2}-\mathrm{CH}_{2}-\underline{\mathrm{CH}_{3}}\right)$ ppm. MS (ES, +ve mode) $: \mathrm{m} / \mathrm{z}$ (abundance) $=139(100)[\mathrm{bmim}]^{+}$. The characterization data for [bmim]Cl were in good agreement with those reported in the literature. ${ }^{\mathrm{S} 1}$ 


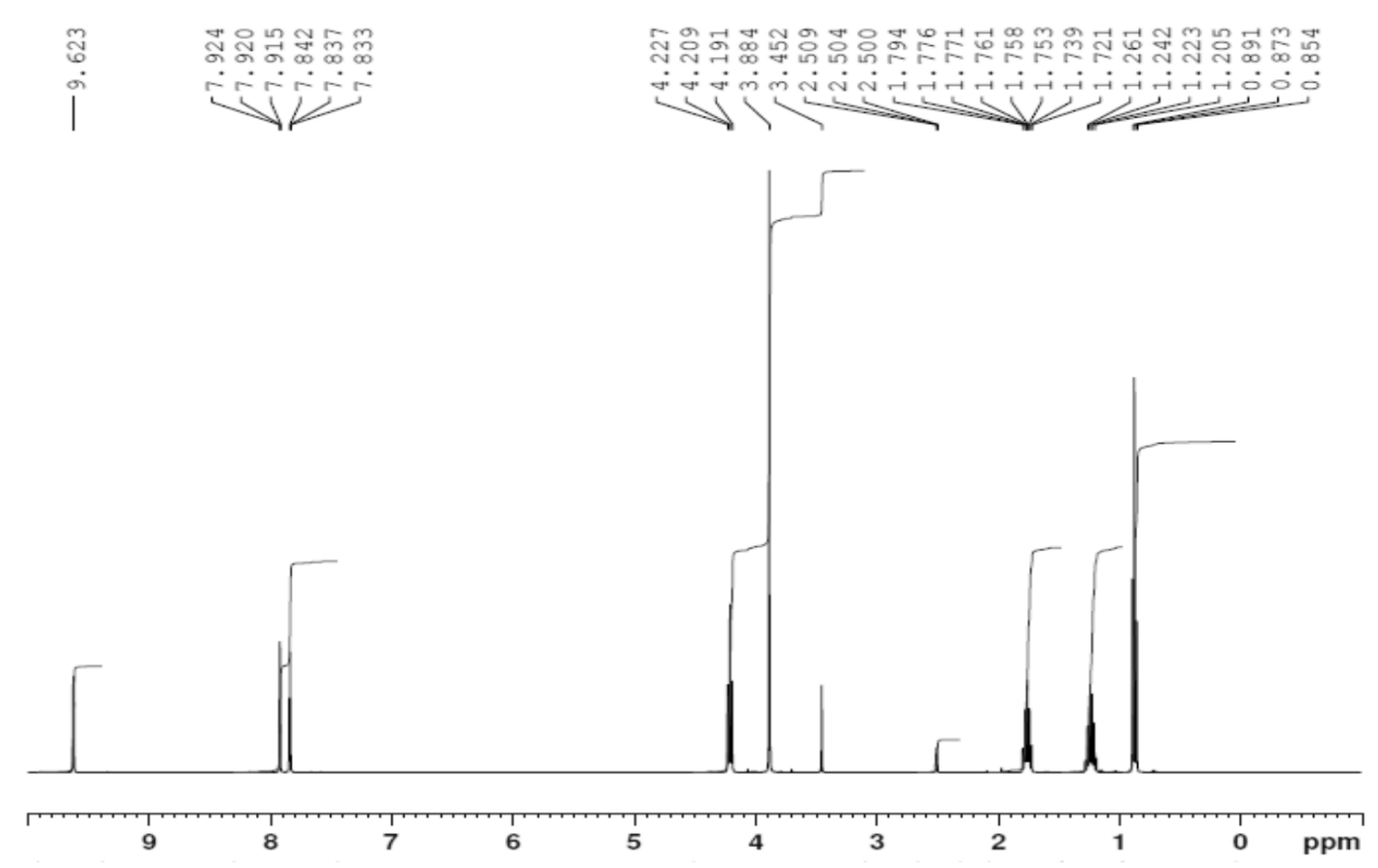

Figure S1. ${ }^{1} \mathrm{H}$ NMR spectrum of [bmim]Cl in deuterated DMSO.

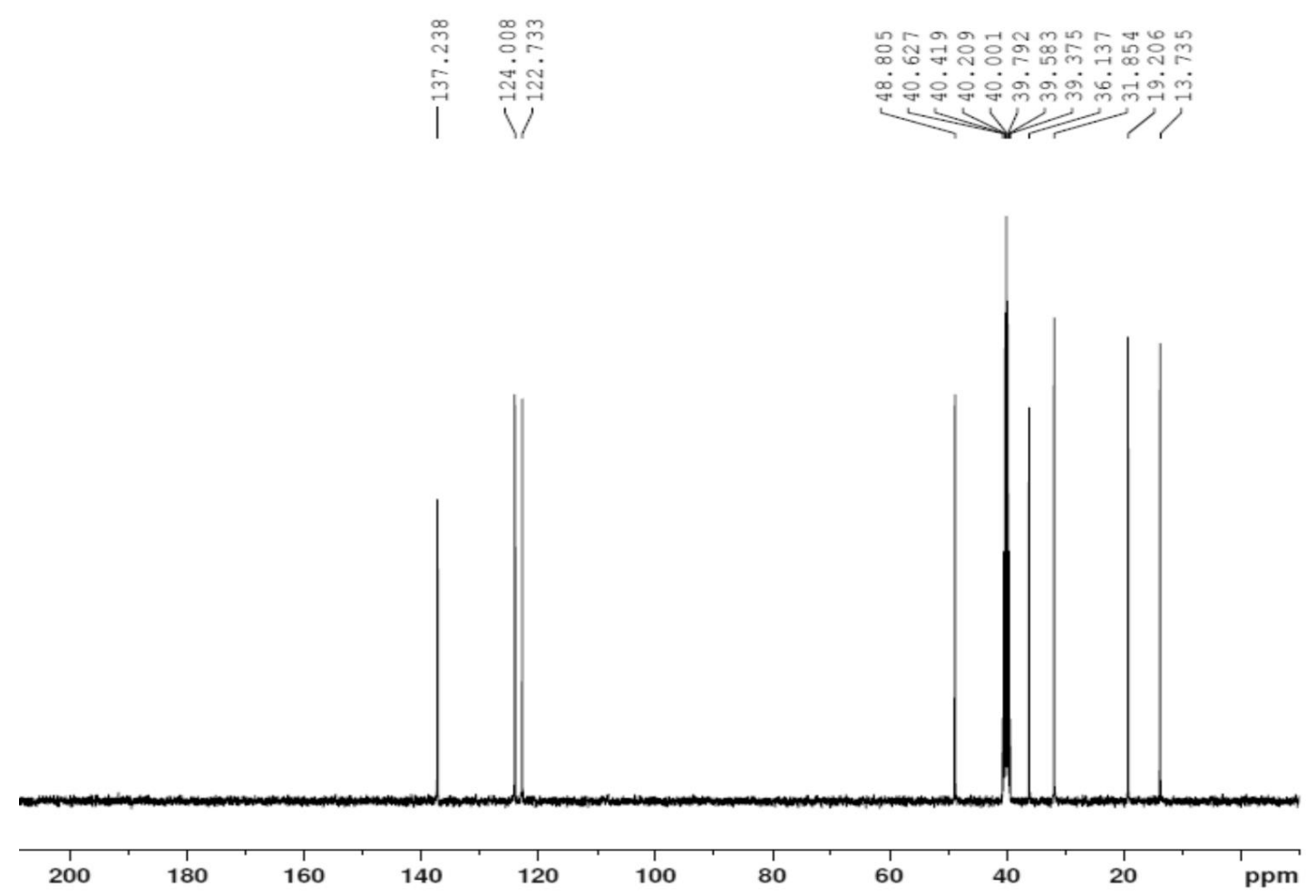

Figure S2. ${ }^{13} \mathrm{C}\left\{{ }^{1} \mathrm{H}\right\}$ NMR spectrum of $[\mathrm{bmim}] \mathrm{Cl}$ in deuterated DMSO. 


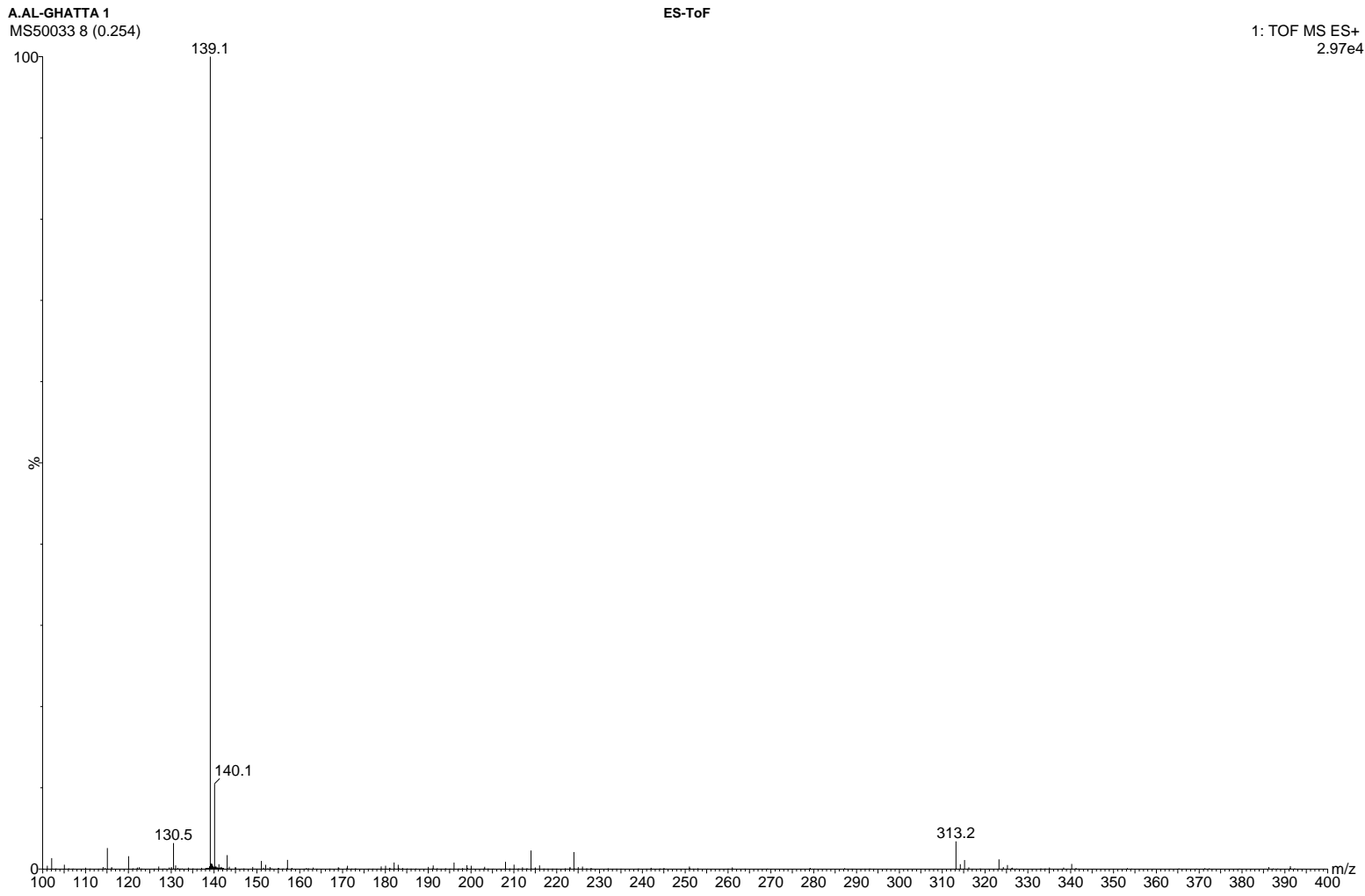

Figure S3. Mass spectrum of [bmim]Cl. 


\section{S2.2 Synthesis of [bmim]Br}

The ionic liquid [bmim] $\mathrm{Br}$ was synthesized by the same procedure as employed for [bmim]Cl but using 1-bromobutane in place of 1-chlorobutane.

[bmim]Br: ${ }^{1} \mathrm{H}$ NMR (DMSO-d $\left.\mathrm{d}_{6}\right): \delta 9.33\left(\mathrm{~s}, 1 \mathrm{H}, \mathrm{N}_{2} \mathrm{CH}\right), 7.86,7.78(\mathrm{~m} \times 2,2 \times 1 \mathrm{H}, \mathrm{N}-\mathrm{CH}), 4.20$ (t, $\left.2 \mathrm{H}, \mathrm{N}-\mathrm{CH}_{2}-\mathrm{CH}_{2}, \mathrm{~J}_{\mathrm{HH}}=7.2 \mathrm{~Hz}\right), 3.87\left(\mathrm{~s}, 3 \mathrm{H}, \mathrm{N}-\mathrm{CH}_{3}\right), 1.76,1.25\left(\mathrm{~m} \times 2,2 \times 2 \mathrm{H}, \mathrm{N}-\mathrm{CH}_{2}-\mathrm{C}_{2}-\right.$ $\left.\mathrm{C}_{2}-\mathrm{CH}_{3}\right), 0.88\left(\mathrm{t}, 3 \mathrm{H},-\mathrm{CH}_{2}-\mathrm{C}_{3}, J_{\mathrm{HH}}=7.3 \mathrm{~Hz}\right) \mathrm{ppm} .{ }^{13} \mathrm{C}\left\{{ }^{1} \mathrm{H}\right\} \mathrm{NMR}\left(\mathrm{DMSO}-\mathrm{d}_{6}\right): \delta 137.2\left(\mathrm{~N}_{2}-\right.$ $\mathrm{CH}), \quad 124.1, \quad 122.7(2 \times \quad \mathrm{N}-\mathrm{CH}), \quad 48.8 \quad\left(\mathrm{~N}-\mathrm{CH}_{2}-\mathrm{CH}_{2}-\mathrm{CH}_{2}-\mathrm{CH}_{3}\right), \quad 36.1 \quad\left(\mathrm{NCH}_{3}\right), \quad 31.8$ $\left(\mathrm{NCH}_{2} \underline{\mathrm{C}} \mathrm{H}_{2} \mathrm{CH}_{2} \mathrm{CH}_{3}\right), 19.2\left(\mathrm{NCH}_{2}-\mathrm{CH}_{2}-\underline{\mathrm{CH}_{2}}-\mathrm{CH}_{3}\right), 13.7\left(\mathrm{~N}-\left(\mathrm{CH}_{2}\right)_{2}-\mathrm{CH}_{2}-\underline{\mathrm{C}} \mathrm{H}_{3}\right)$ ppm. MS (ES, +ve mode) $: m / z$ (abundance) $=357(31)\left[(\mathrm{bmim})_{2} \mathrm{Br}\right]^{+}, 139(100)[\mathrm{bmim}]^{+}$. The characterization data for $\left[\right.$ bmim] $\mathrm{Cl}$ were in good agreement with those reported in the literature. ${ }^{\mathrm{S} 2}$

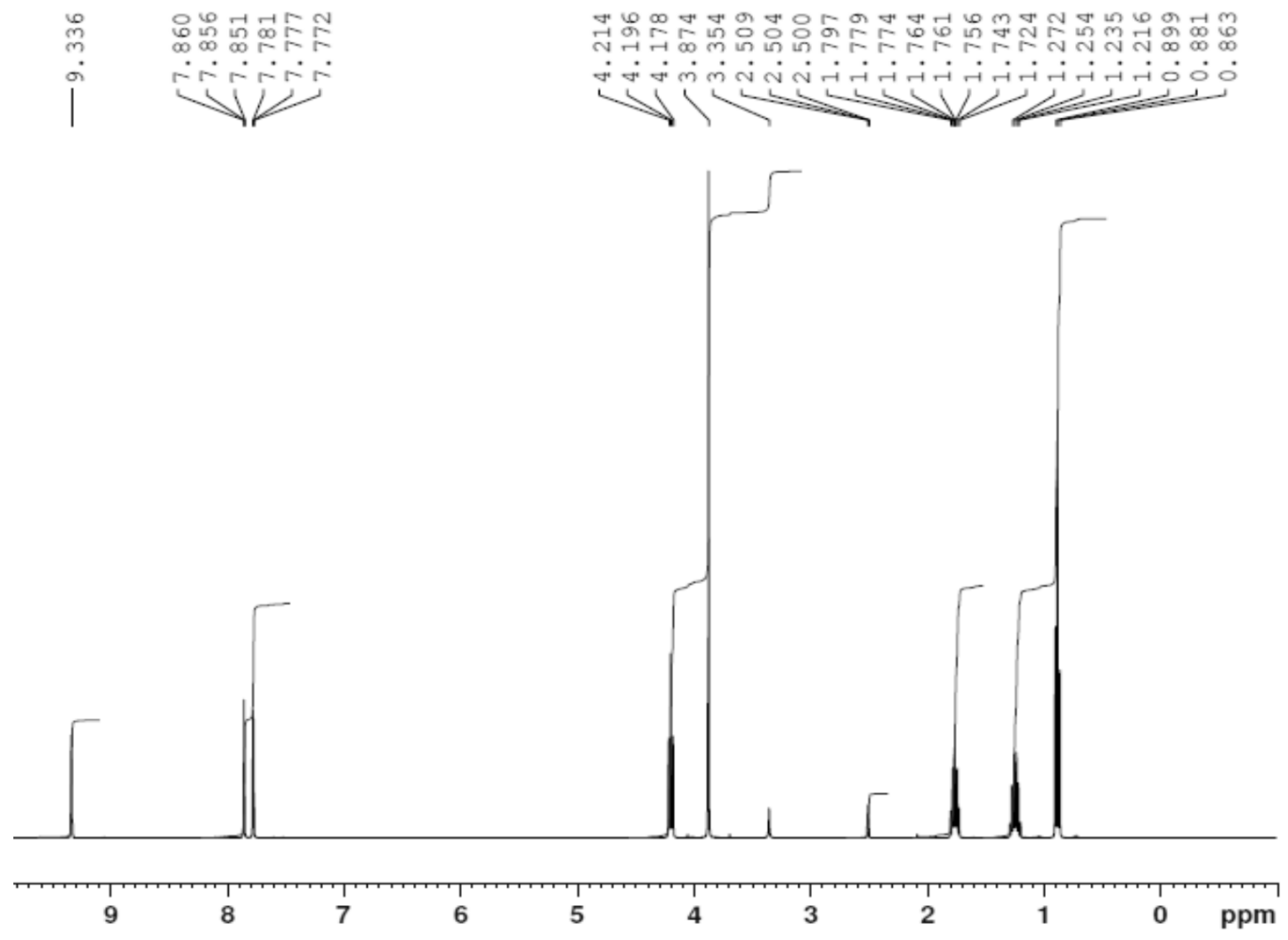

Figure S4. ${ }^{1} \mathrm{H}$ NMR spectrum of [bmim]Br in deuterated DMSO. 


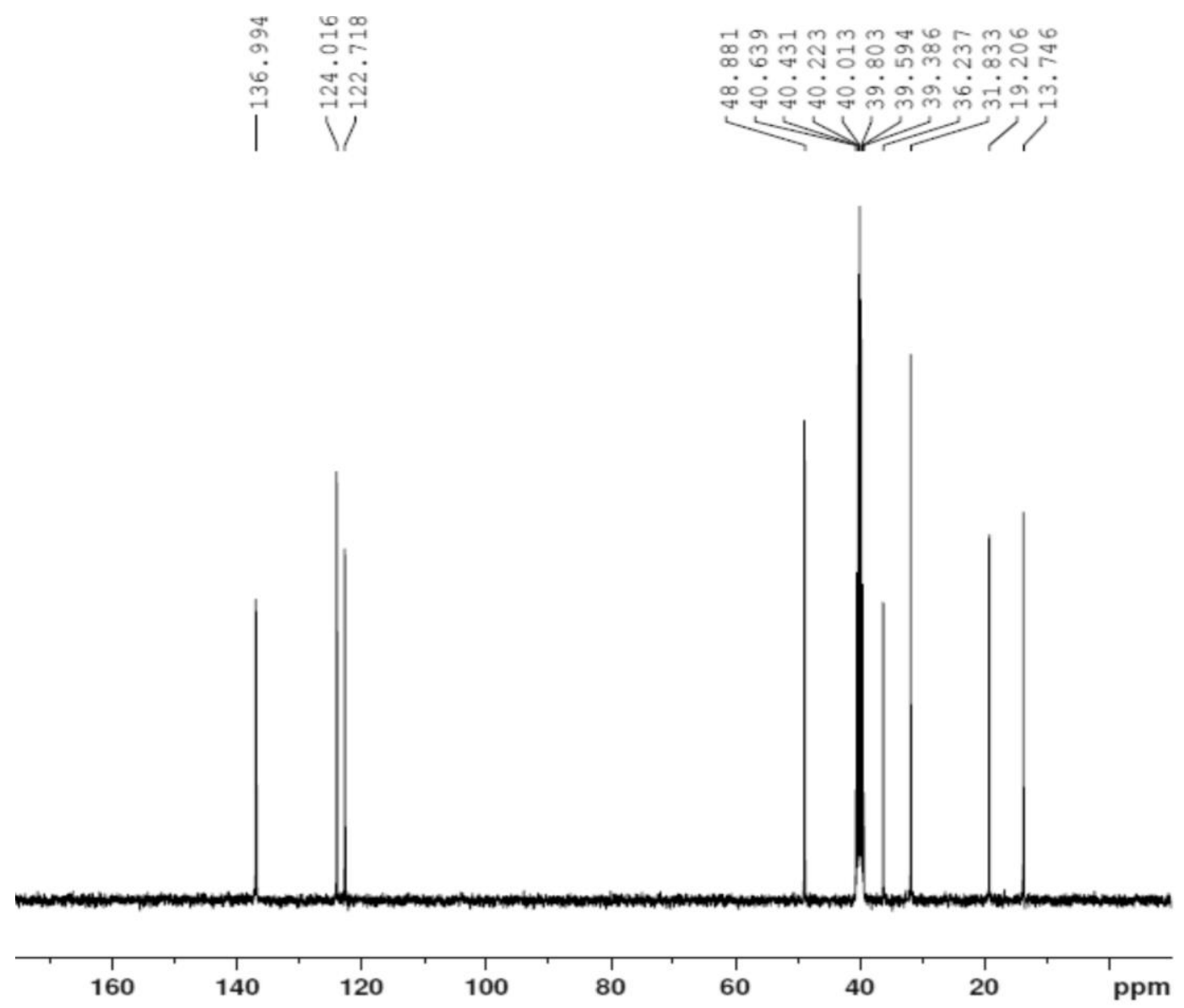

Figure S5. ${ }^{13} \mathrm{C}\left\{{ }^{1} \mathrm{H}\right\}$ NMR spectrum of [bmim]Br in deuterated DMSO.

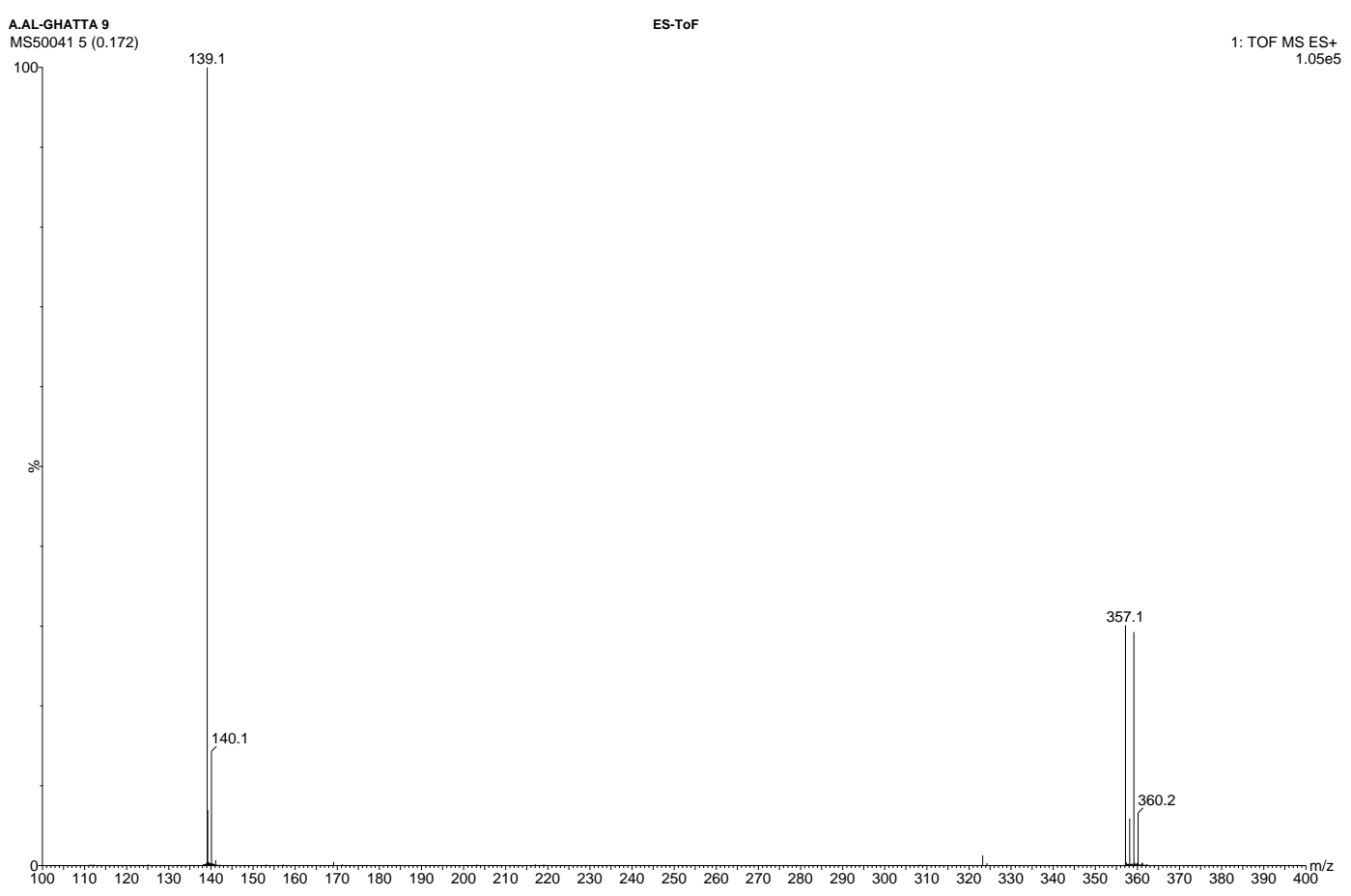

Figure S6. Mass spectrum of [bmim] Br. 


\section{S2.3 Synthesis of [bmpyr]Cl}

The ionic liquid [bmpyr] $\mathrm{Cl}$ was prepared in the same way as $[\mathrm{bmim}] \mathrm{Cl}$ but using $\mathrm{N}$ methylpyrrolidine in place of 1-methylimidazole.

[bmpyr]Cl: ${ }^{1} \mathrm{H}$ NMR (DMSO- $\left.\mathrm{d}_{6}\right)$ : 3.58-3.39 (m, 6H, 2,5-pyrrole- $\mathrm{CH}_{2}$ and $\mathrm{N}-\mathrm{CH}_{2}-\mathrm{CH}_{2}-\mathrm{CH}_{2}-$ $\mathrm{CH}_{3}$ ), $3.03\left(\mathrm{~s}, 3 \mathrm{H}, \mathrm{N}-\mathrm{CH}_{3}\right), 2.02-2.05\left(\mathrm{~m}, 2 \times 2 \mathrm{H}, 3,4-\right.$ pyrrole- $\left.\mathrm{CH}_{2}\right), 1.65(\mathrm{~m}, 2 \mathrm{H}$, $\left.\mathrm{NCH}_{2} \mathrm{C}_{2} \mathrm{CH}_{2} \mathrm{CH}_{3}, J_{\mathrm{HH}}=7.2 \mathrm{~Hz}\right), 1.2\left(\mathrm{~m}, 2 \mathrm{H}, \mathrm{NCH}_{2} \mathrm{CH}_{2} \mathrm{C}_{2} \mathrm{CH}_{3}, J_{\mathrm{HH}}=7.3 \mathrm{~Hz}\right), 0.86(\mathrm{t}, 3 \mathrm{H}, \mathrm{N}-$ $\left.\mathrm{CH}_{2}-\mathrm{CH}_{2}-\mathrm{CH}_{2}-\underline{\mathrm{C}}_{3}, \mathrm{~J}_{\mathrm{HH}}=7.3 \mathrm{~Hz}\right)$ ppm. ${ }^{13} \mathrm{C}\left\{{ }^{1} \mathrm{H}\right\} \mathrm{NMR}\left(\mathrm{DMSO}-\mathrm{d}_{6}\right): 63.7$ (s, N- $\mathrm{CH}_{2}-\mathrm{CH}_{2}$ ), 63.0

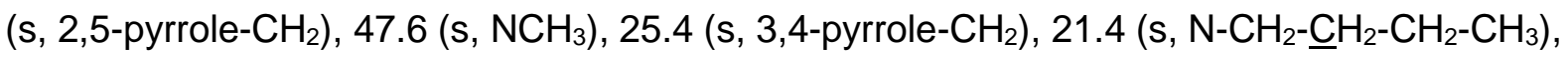

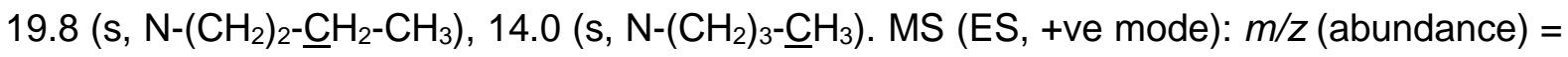
142(100) [bmpyr] $]^{+}$. The characterization data for [bmpyr]Cl were in good agreement with those reported in the literature. ${ }^{\text {S3 }}$

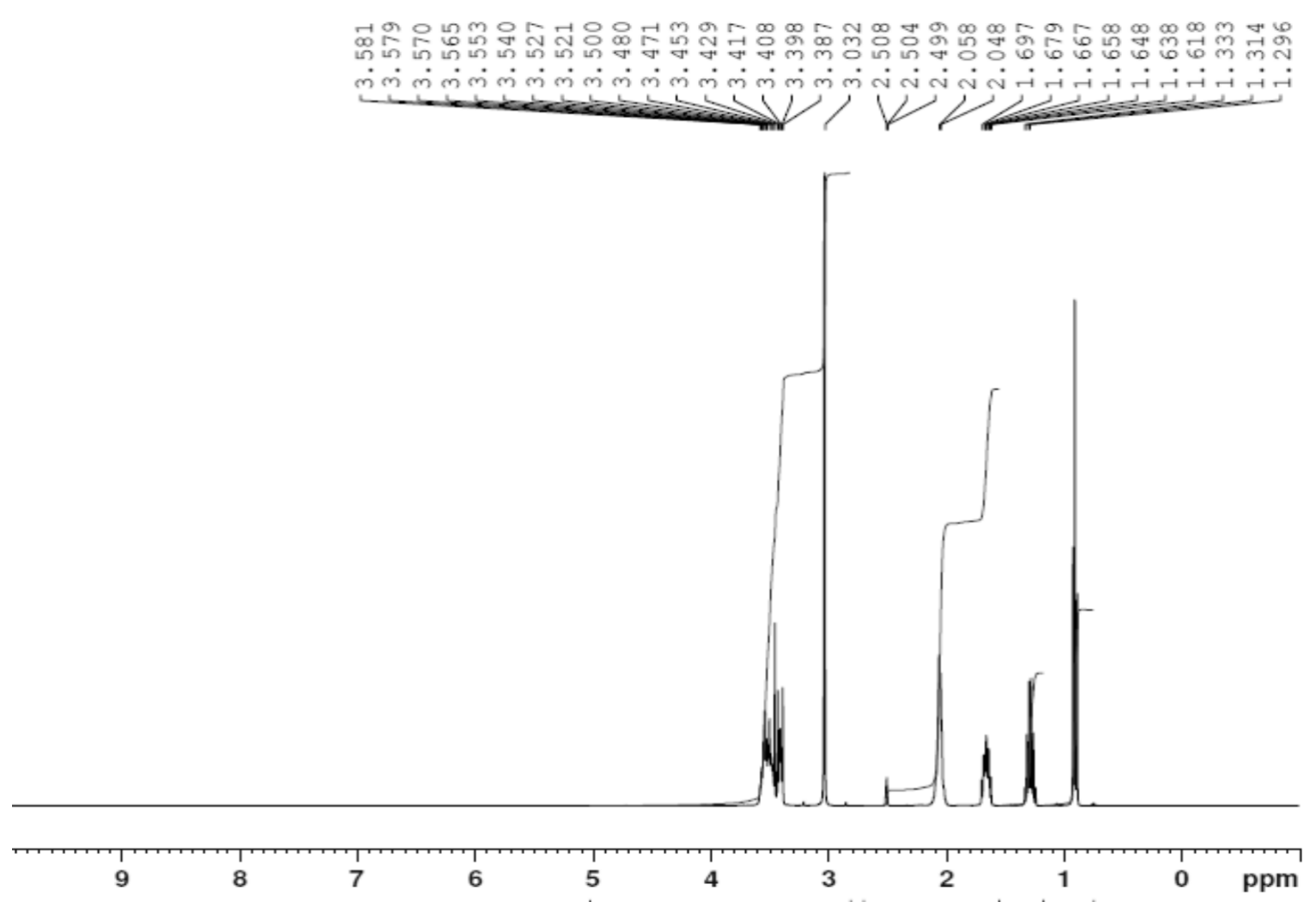

Figure S7. ${ }^{1} \mathrm{H}$ NMR spectrum of [bmpyr]Cl in deuterated DMSO. 


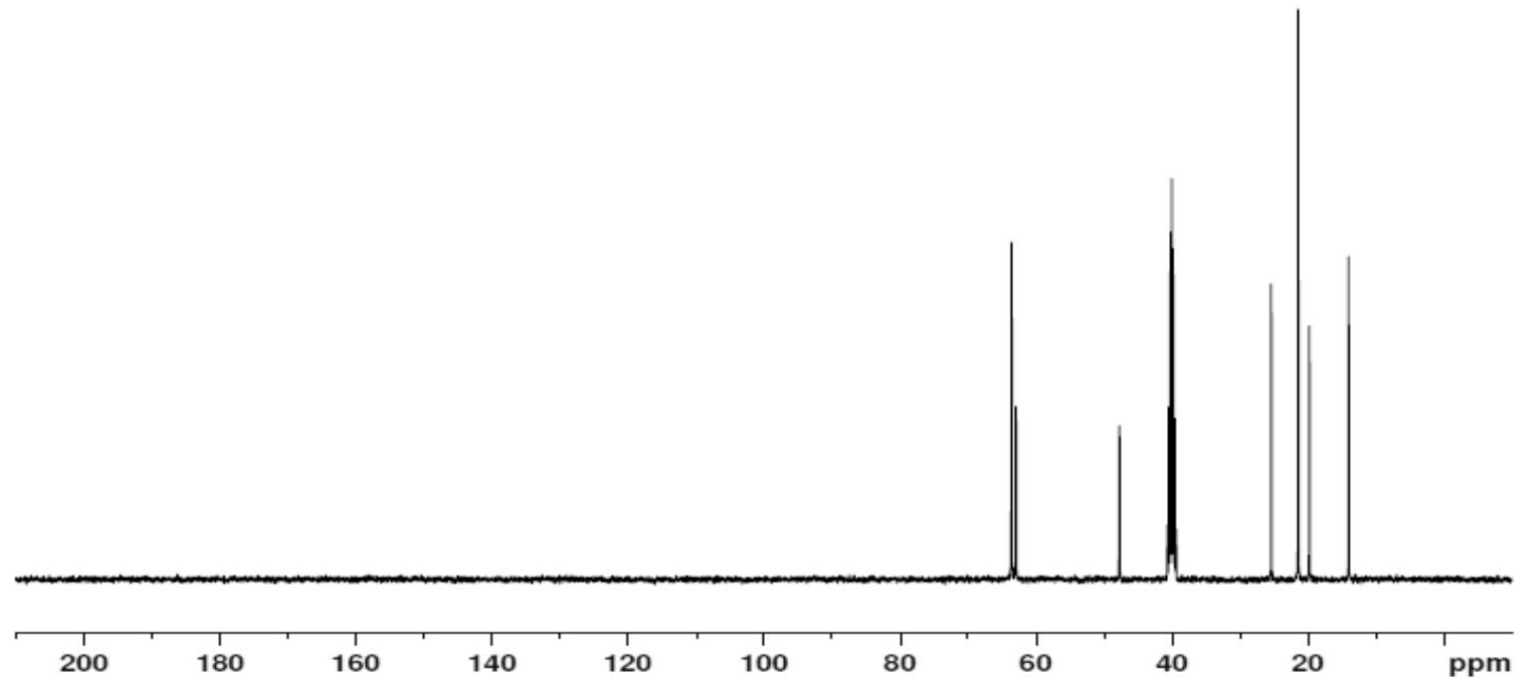

Figure S8. ${ }^{13} \mathrm{C}\left\{{ }^{1} \mathrm{H}\right\}$ NMR spectrum of [bmpyr]Cl in deuterated DMSO.

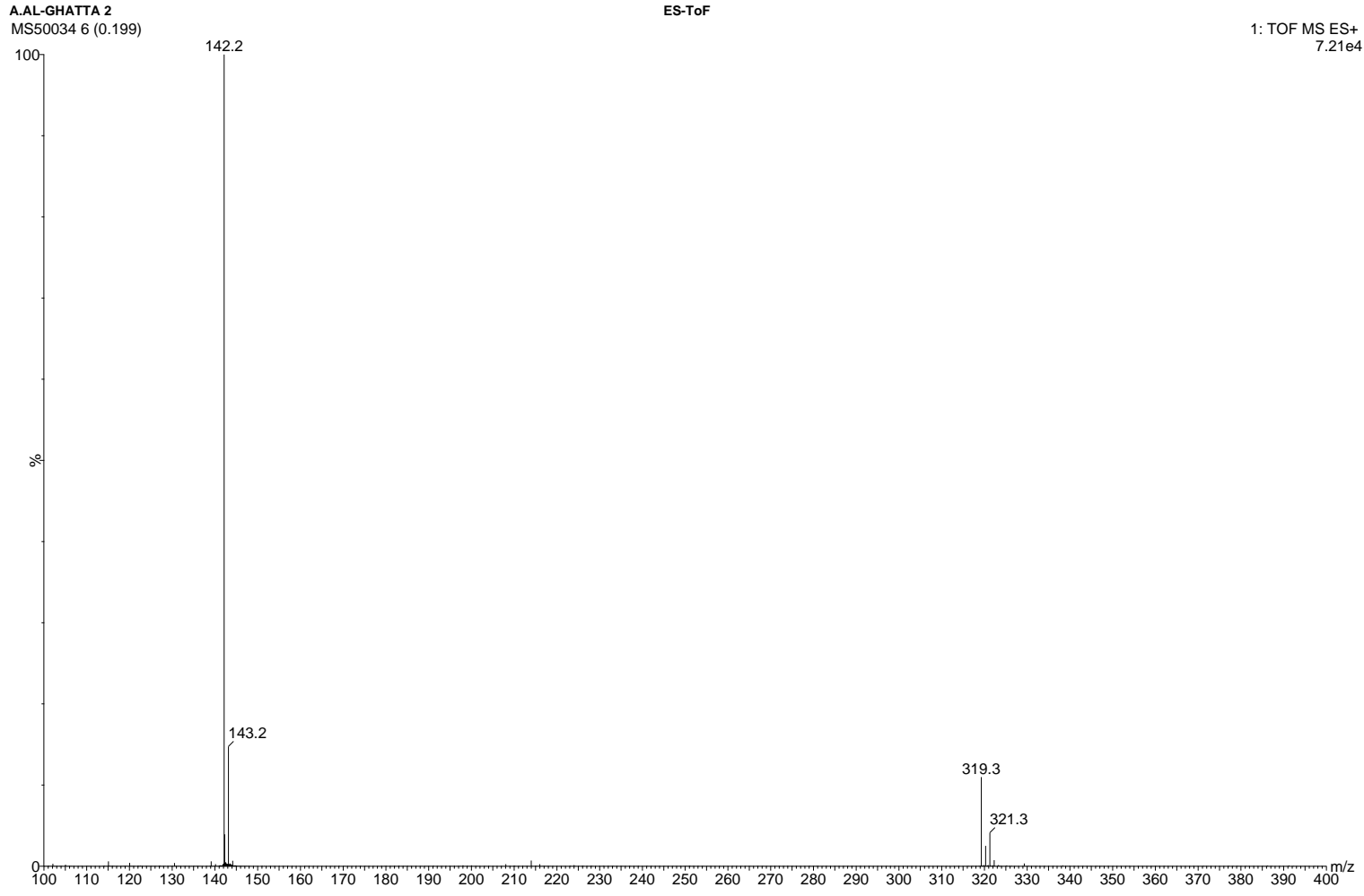

Figure S9. Mass spectrum of [bmpyr]Cl. 


\section{S3 Synthesis of ionic liquids with triflate anions}

\section{S3.1 Synthesis of [bmim][OTf]}

The ionic liquid [bmim]Cl $(30 \mathrm{~g}, 0.17 \mathrm{~mol})$ was dissolved in water $(100 \mathrm{~mL})$ and Li[OTf] $(30 \mathrm{~g}$, $0.179 \mathrm{~mol}, 1.1 \mathrm{eq})$ was dissolved in water $(50 \mathrm{~mL})$. The lithium triflate solution was added dropwise to the chloride solution under vigorous stirring and the reaction left overnight. The product was extracted from aqueous solution using a total of $800 \mathrm{~mL}$ dichloromethane. The organic extracts were washed repeatedly with water $(5 \mathrm{~mL})$ until the silver nitrate test revealed no halide ions (5-6 washes). The organic phase was dried with magnesium sulfate and then evaporated to dryness under reduced pressure. The ionic liquid, [bmim][OTf] was further dried under reduced pressure overnight at $60^{\circ} \mathrm{C}$ (final yield: $47 \mathrm{~g}, 96 \%$ ) and handled thereafter under an inert atmosphere. The characterization data for [bmim] $\mathrm{Cl}$ were in good agreement with those reported in the literature. ${ }^{\mathrm{S}}$

[bmim][OTf]: ${ }^{1} \mathrm{H}$ NMR (DMSO-d $\left.\mathrm{d}_{6}\right): \delta 9.08\left(\mathrm{~s}, 1 \mathrm{H}, \mathrm{N}_{2} \mathrm{CH}\right), 7.76-7.69-(\mathrm{m} \times 2,2 \times 1 \mathrm{H}, \mathrm{N}-\mathrm{CH})$, $4.17\left(\mathrm{t}, 2 \mathrm{H}, \mathrm{N}-\mathrm{C}_{2}-\mathrm{CH}_{2}, \mathrm{~J}_{\mathrm{HH}}=7.2 \mathrm{~Hz}\right), 3.85\left(\mathrm{~s}, 3 \mathrm{H}, \mathrm{N}-\mathrm{CH}_{3}\right), 1.77-1.26\left(\mathrm{~m} \times 2,2 \times 2 \mathrm{H}, \mathrm{N}-\mathrm{CH}_{2}-\right.$ $\mathrm{C}_{2}-\underline{\mathrm{C}}_{2}-\mathrm{CH}_{3}$ ), 0.9 (t, 3H, $-\mathrm{CH}_{2}-\underline{\mathrm{H}}_{3}, \mathrm{~J}_{\mathrm{HH}}=7.4 \mathrm{~Hz}$ ) ppm. ${ }^{13} \mathrm{C}\left\{{ }^{1} \mathrm{H}\right\}$ NMR (DMSO-d 6 ): $\delta 137.7$ $\left(\mathrm{N}_{2}-\mathrm{CH}\right), 123.6-122.4(2 \times \mathrm{N}-\mathrm{CH}), 119.2\left(\mathrm{CF}_{3}\right), 49.1\left(\mathrm{~N}-\underline{\mathrm{C}} \mathrm{H}_{2}-\mathrm{CH}_{2}-\mathrm{CH}_{2}-\mathrm{CH}_{3}\right), 35.8\left(\mathrm{NCH}_{3}\right), 32.0$ $\left(\mathrm{NCH}_{2} \underline{\mathrm{CH}_{2}} \mathrm{CH}_{2} \mathrm{CH}_{3}\right), 19.0\left(\mathrm{NCH}_{2}-\mathrm{CH}_{2}-\underline{\mathrm{CH}_{2}}-\mathrm{CH}_{3}\right), 13.8\left(\mathrm{~N}-\left(\mathrm{CH}_{2}\right)_{2}-\mathrm{CH}_{2}-\underline{\mathrm{CH}_{3}}\right)$ ppm. MS (ES, +ve mode) $: m / z$ (abundance) $=139(100)[\mathrm{bmim}]^{+}$. The characterization data for [bmpyr]Cl were in good agreement with those reported in the literature. ${ }^{S 4}$

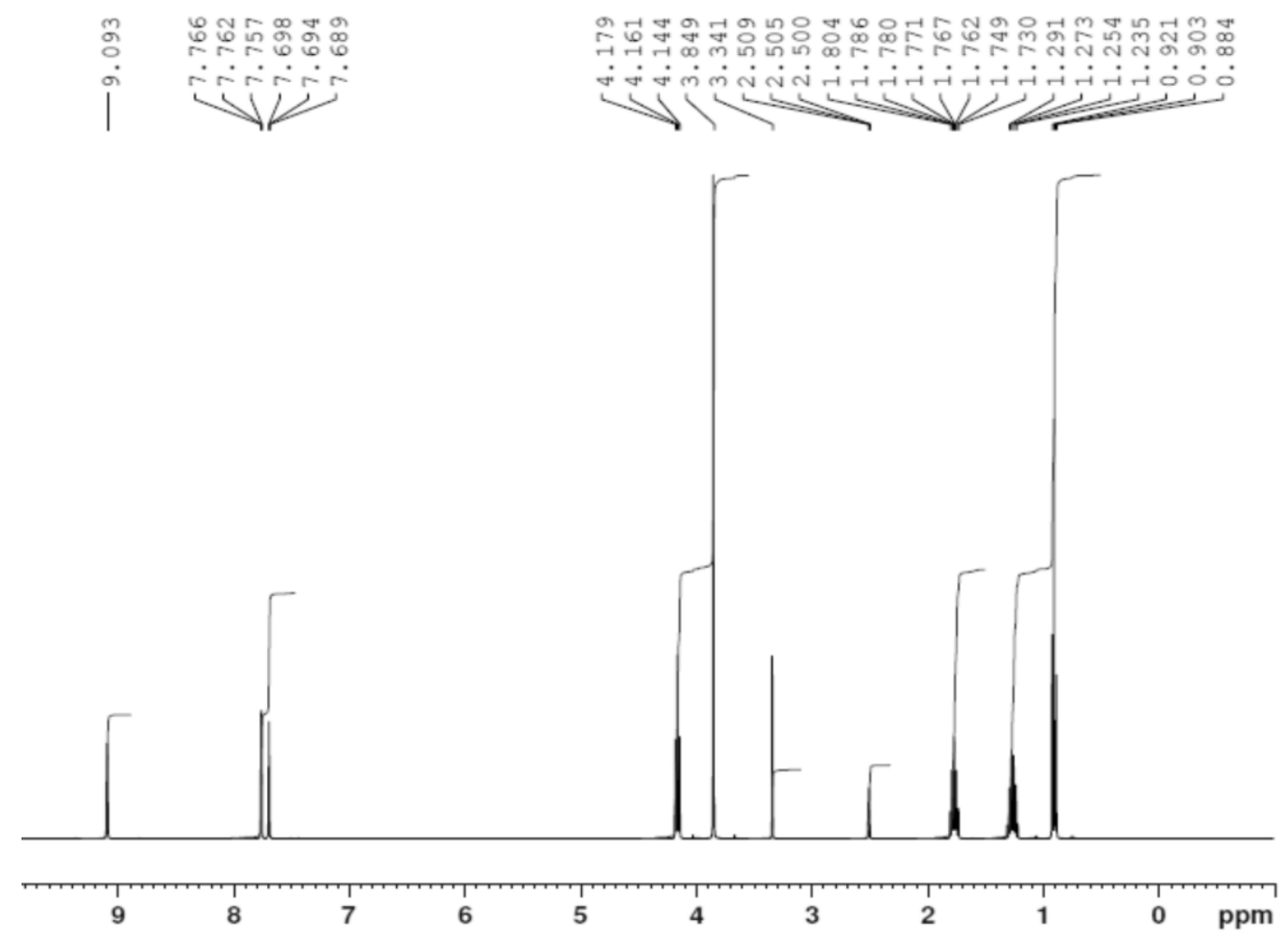

Figure S10. ${ }^{1} \mathrm{H}$ NMR spectrum of [bmim][OTf] in deuterated DMSO. 


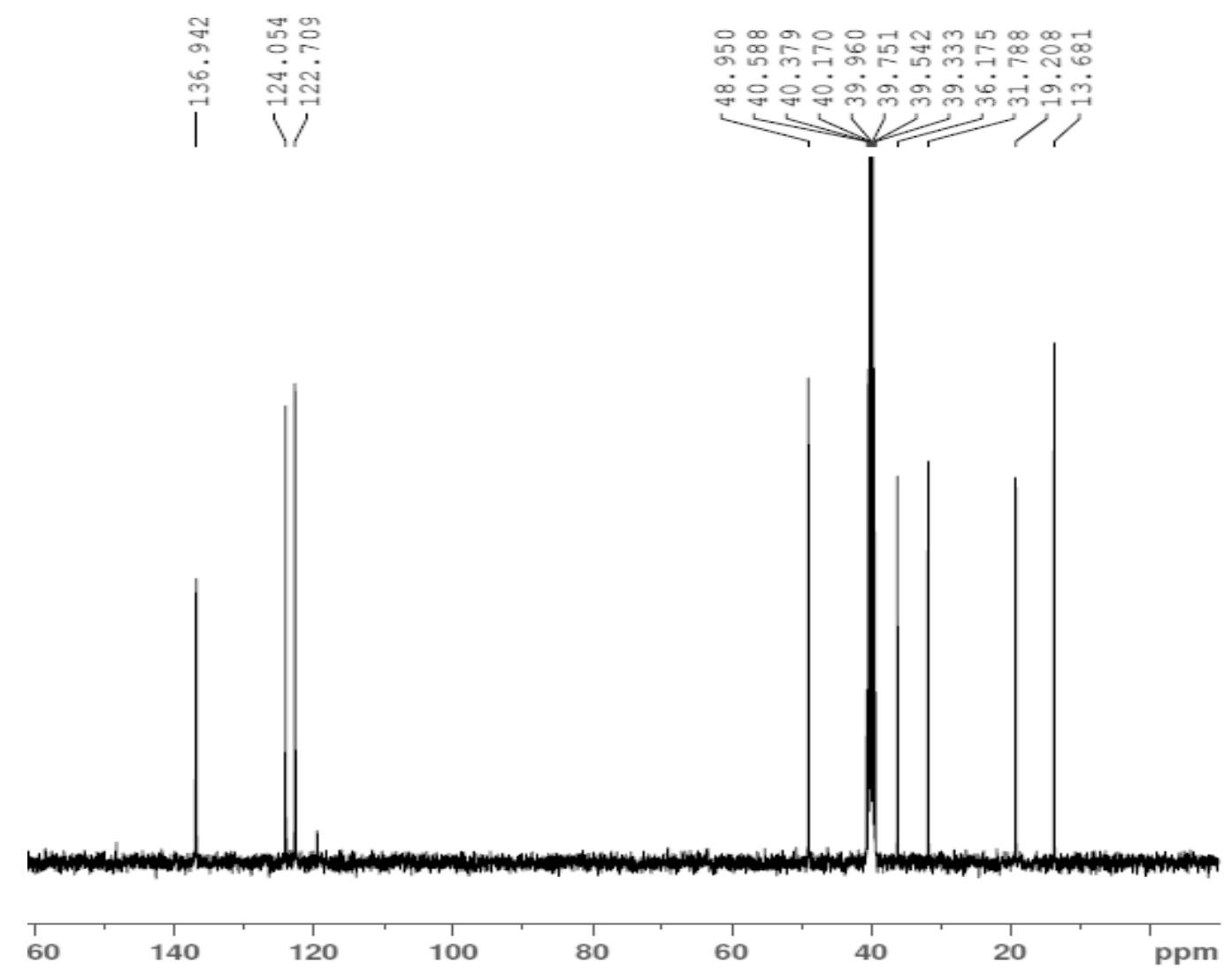

Figure $\mathrm{S} 11 .{ }^{13} \mathrm{C}\left\{{ }^{1} \mathrm{H}\right\}$ NMR spectrum of [bmim][OTf] in deuterated DMSO.

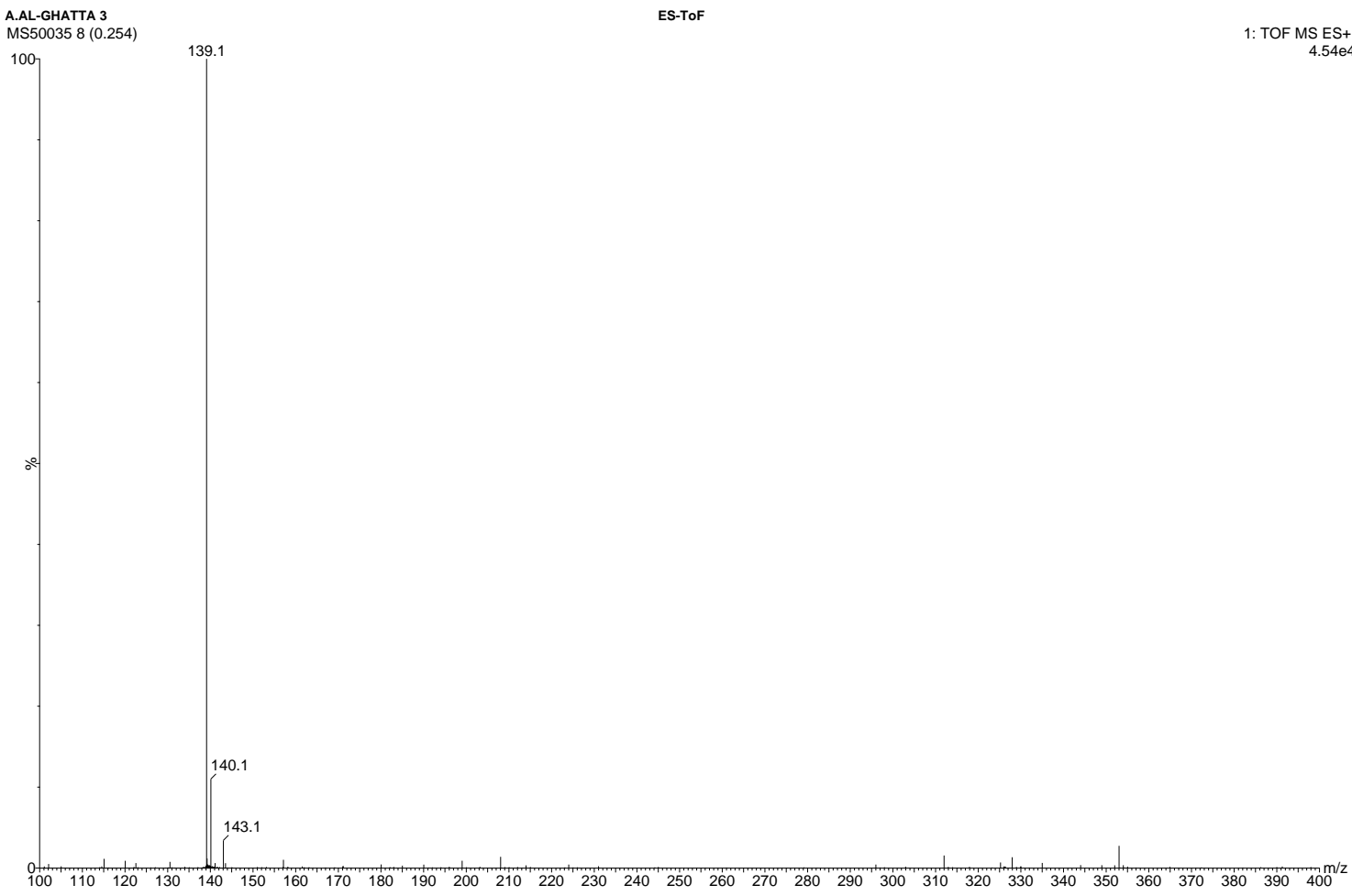

Figure S12. Mass spectrum of [bmim][OTf]. 


\section{S3.2 Synthesis of [bmpyr][OTf]}

The ionic liquid [bmpyr][OTf] was synthesized by the same procedure as employed for [bmim][OTf] but starting from [bmpyr]Cl.

[bmpyr][OTf]: ${ }^{1} \mathrm{H}$ NMR (DMSO- $\left.\mathrm{d}_{6}\right)$ : 3.51-3.26 (m, 6H, 2,5-pyrrole- $\mathrm{CH}_{2}$ and $\mathrm{N}-\mathrm{CH}_{2}-\mathrm{CH}_{2}-\mathrm{CH}_{2}-$ $\mathrm{CH}_{3}$ ), 2.98 (s, 3H, N-CH ), 2.02-2.08 (m, $2 \times 2 \mathrm{H}, 3,4-$ pyrrole- $\left.\mathrm{CH}_{2}\right), 1.68(\mathrm{~m}, 2 \mathrm{H}$, $\left.\mathrm{NCH}_{2} \underline{\mathrm{H}}_{2} \mathrm{CH}_{2} \mathrm{CH}_{3}, J_{\mathrm{HH}}=7.2 \mathrm{~Hz}\right), 1.32\left(\mathrm{~m}, 2 \mathrm{H}, \mathrm{NCH}_{2} \mathrm{CH}_{2} \mathrm{C}_{2} \mathrm{CH}_{3}, J_{\mathrm{HH}}=7.3 \mathrm{~Hz}\right), 0.94(\mathrm{t}, 3 \mathrm{H}$, $\mathrm{N}-\mathrm{CH}_{2}-\mathrm{CH}_{2}-\mathrm{CH}_{2}-\mathrm{CH}_{3}, J_{\mathrm{HH}}=7.3 \mathrm{~Hz}$ ) ppm. ${ }^{13} \mathrm{C}\left\{{ }^{1} \mathrm{H}\right\} \mathrm{NMR}\left(\mathrm{DMSO}-\mathrm{d}_{6}\right): 119.4\left(\mathrm{CF}_{3}\right), 63.9$ (s, N$\underline{\mathrm{CH}}_{2}-\mathrm{CH}_{2}$ ), 63.3 (s, 2,5-pyrrole- $\mathrm{CH}_{2}$ ), 47.8 (s, $\mathrm{NCH}_{3}$ ), 25.2 (s, 3,4-pyrrole- $\mathrm{CH}_{2}$ ), 21.5 (s, N-

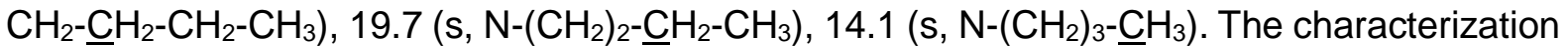
data for $[\mathrm{bmpyr}] \mathrm{Cl}$ were in good agreement with those reported in the literature. ${ }^{\mathrm{S} 5}$

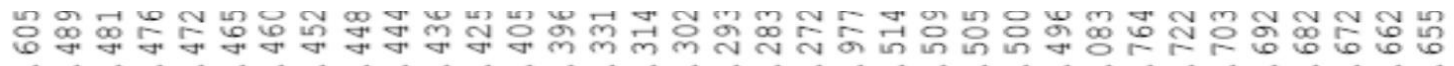

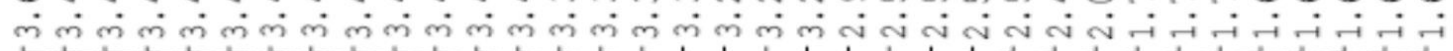

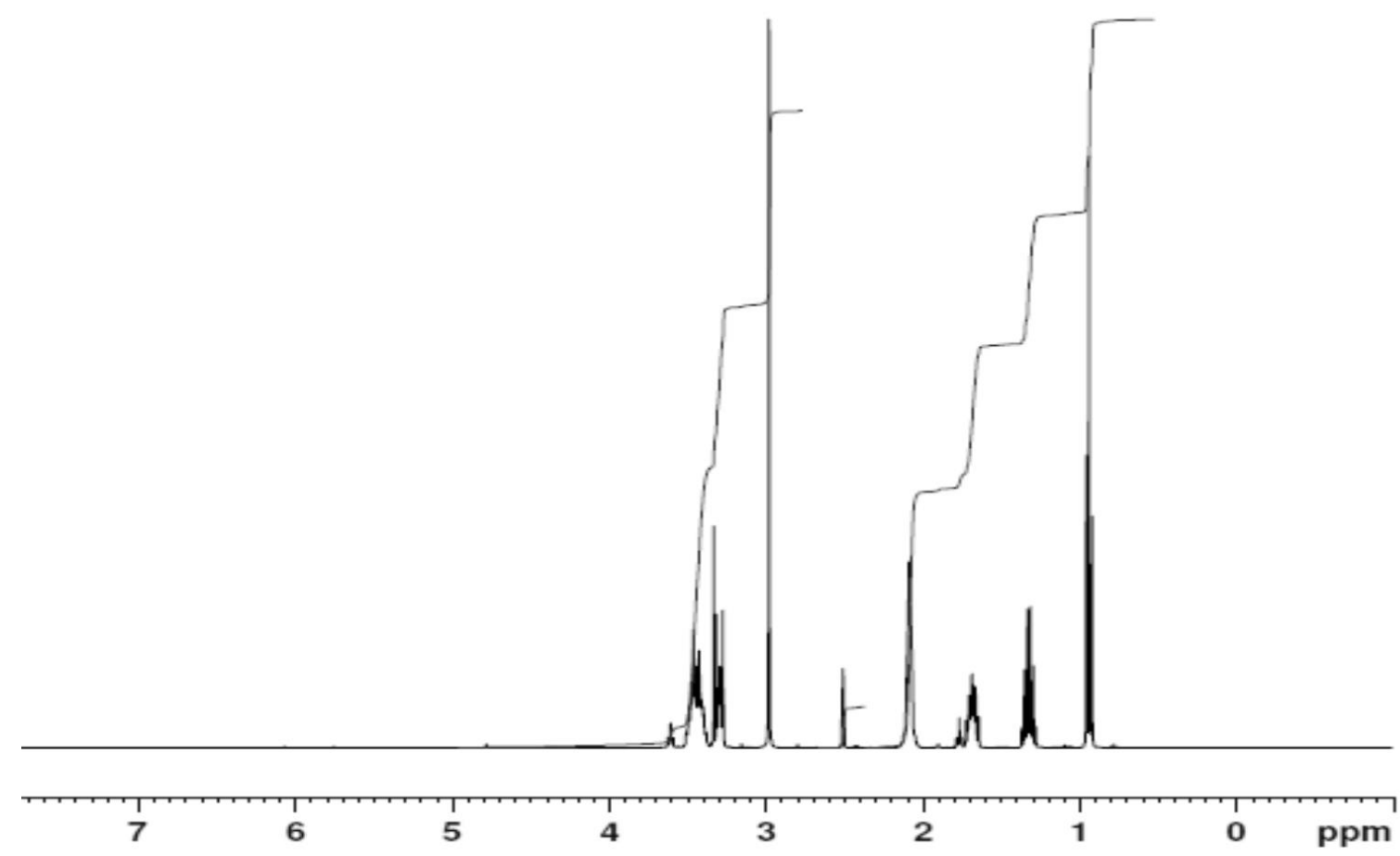

Figure S13. ${ }^{1} \mathrm{H}$ NMR spectrum of [bmpyr][OTf] in deuterated DMSO. 


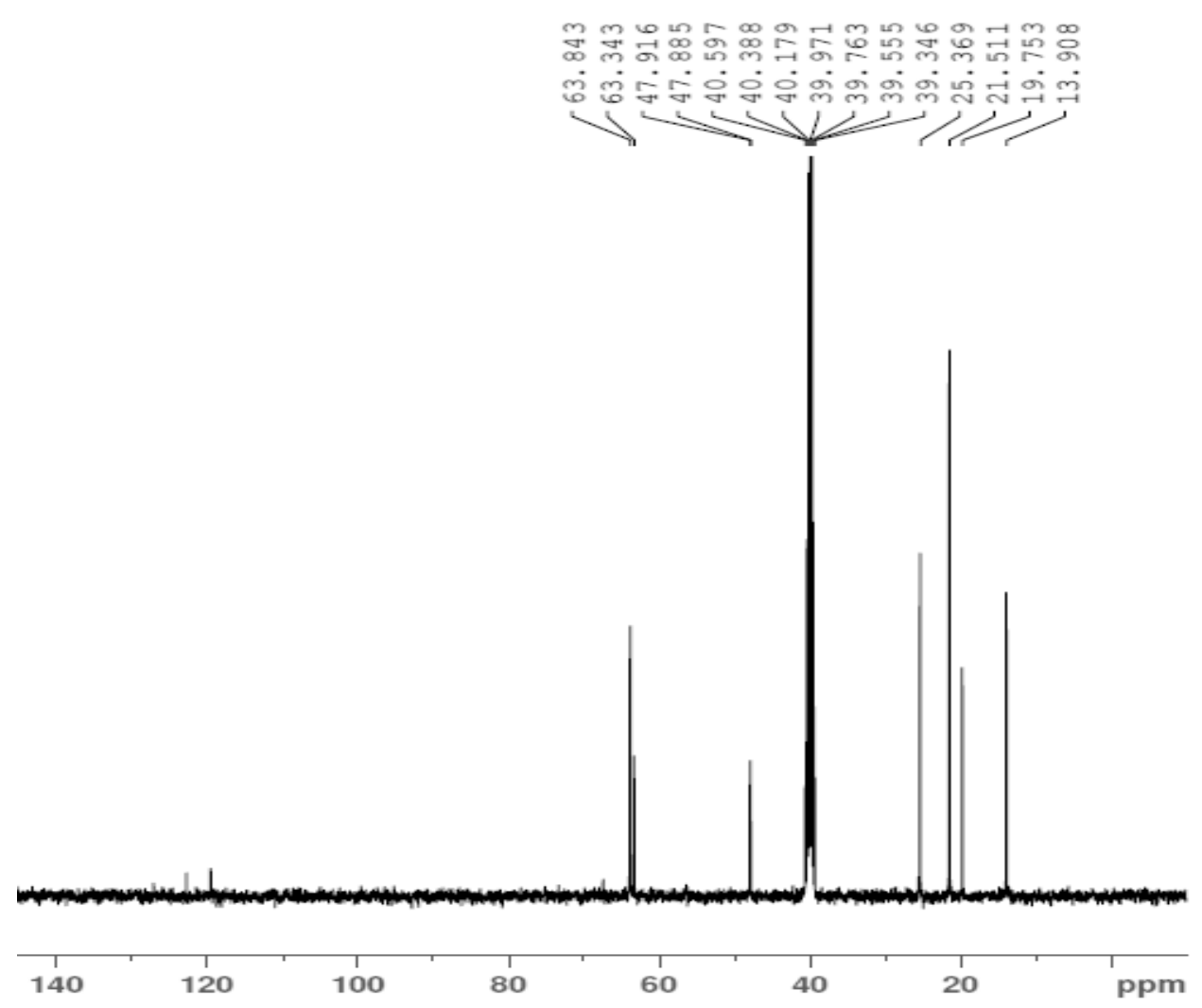

Figure $\mathrm{S} 14 .{ }^{13} \mathrm{C}\left\{{ }^{1} \mathrm{H}\right\}$ NMR spectrum of [bmpyr][OTf] in deuterated DMSO.

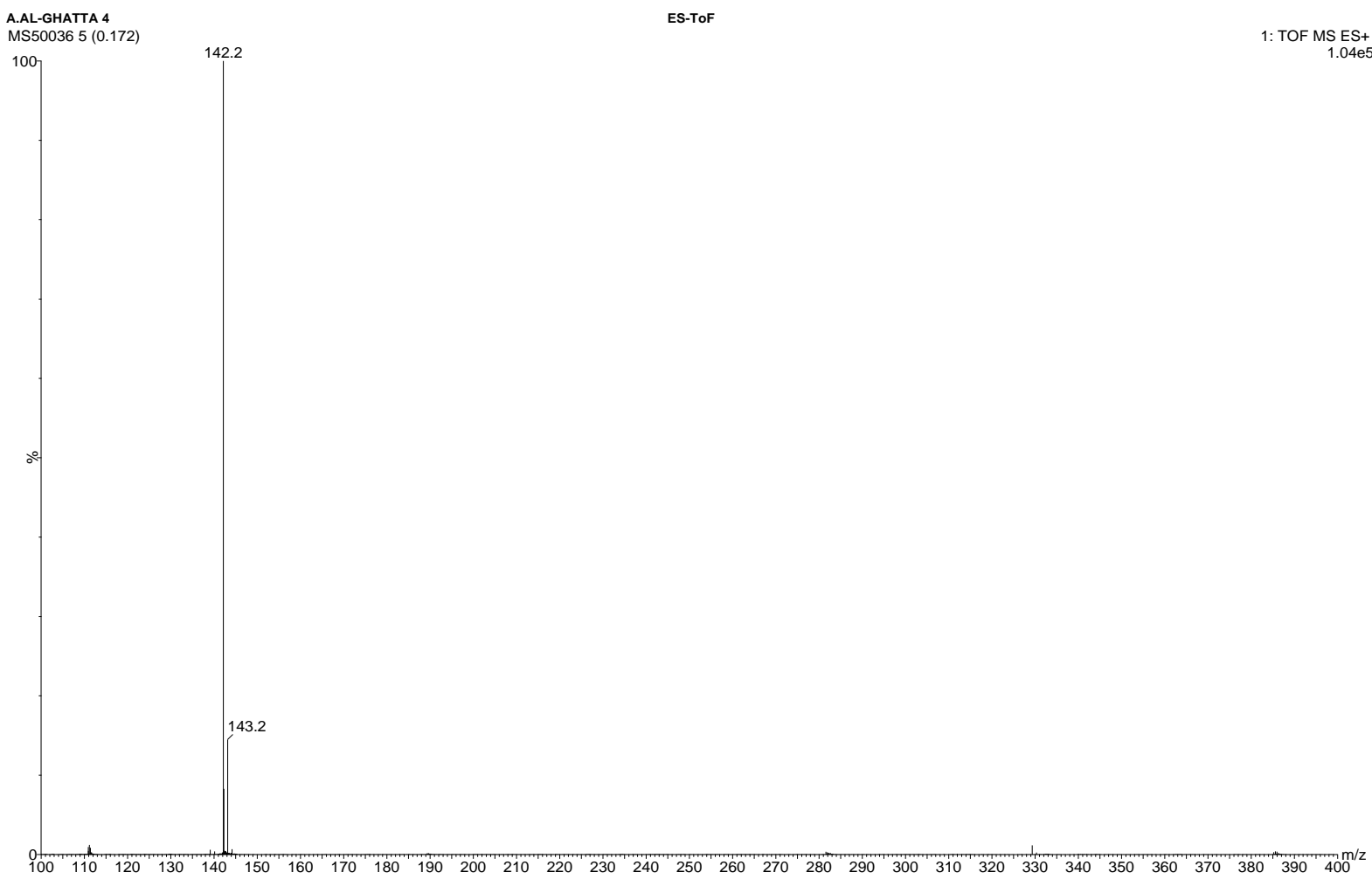

Figure S15. Mass spectrum of [bmpyr][OTf]. 


\section{S4 Mass spectrometry analysis of oligomers}

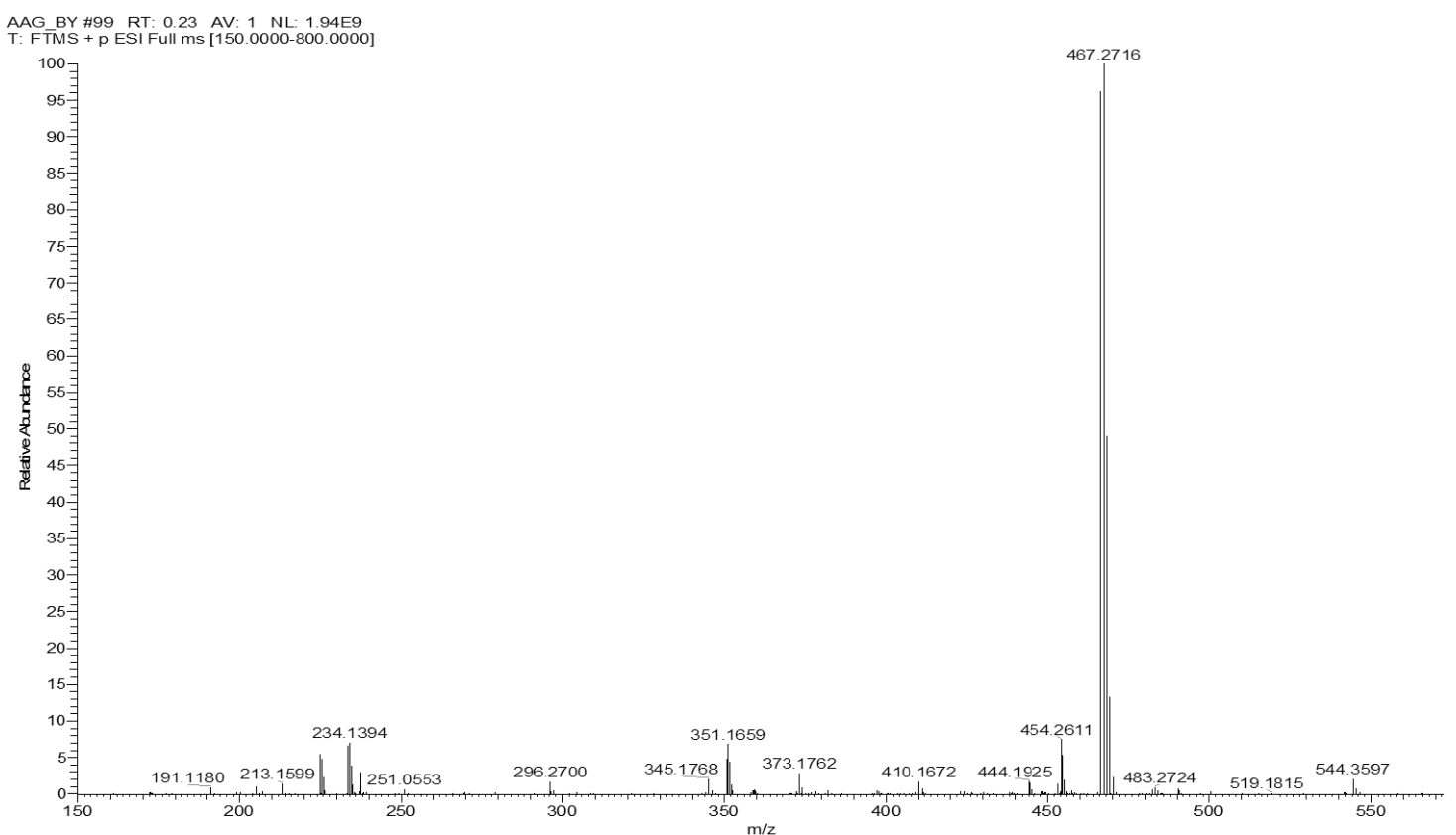

Figure S16. Mass spectrometry of oligomers. Mass at $\mathrm{m} / \mathrm{z} 467.2716$ corresponds to the tetramer unit

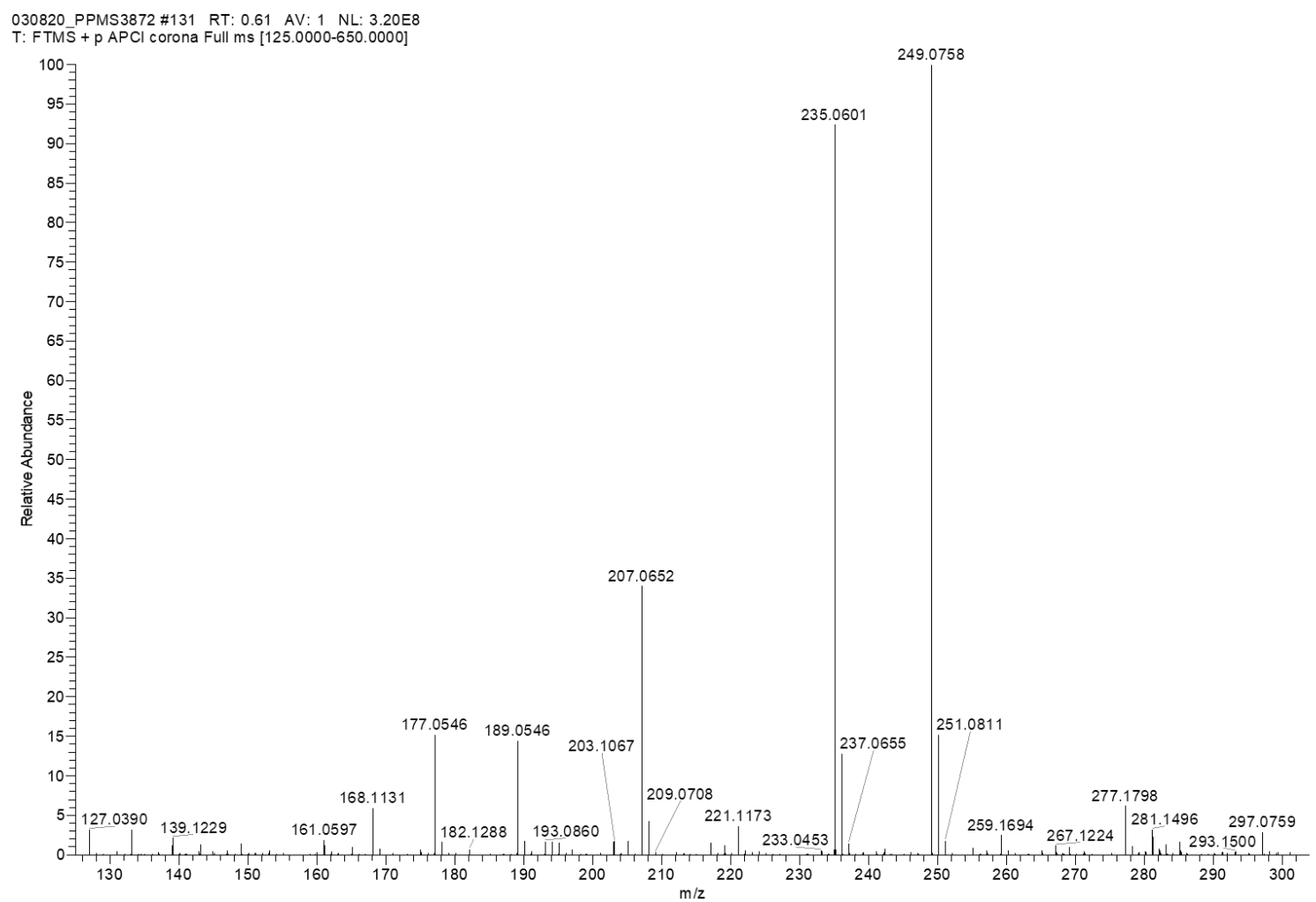

Figure S17. Mass spectrometry of oligomer at lower $\mathrm{m} / \mathrm{z}$ value. Mass at $\mathrm{m} / \mathrm{z} 235.0601$ corresponds to the HMF dimer. 


\section{S5 Scanning Electron Microscopy images of the materials}

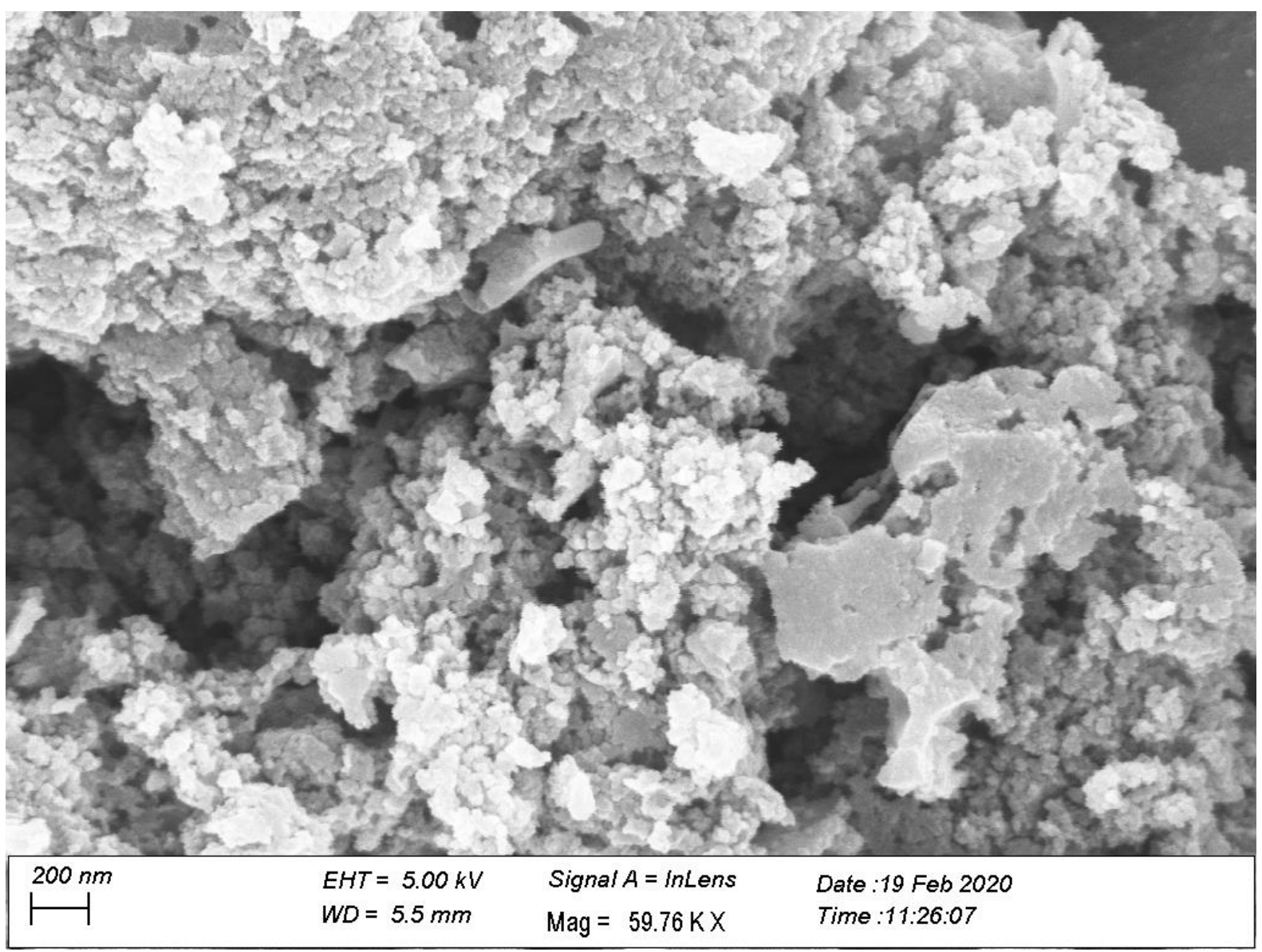

Figure S18. SEM image showing morphology of commercial activated carbon. 


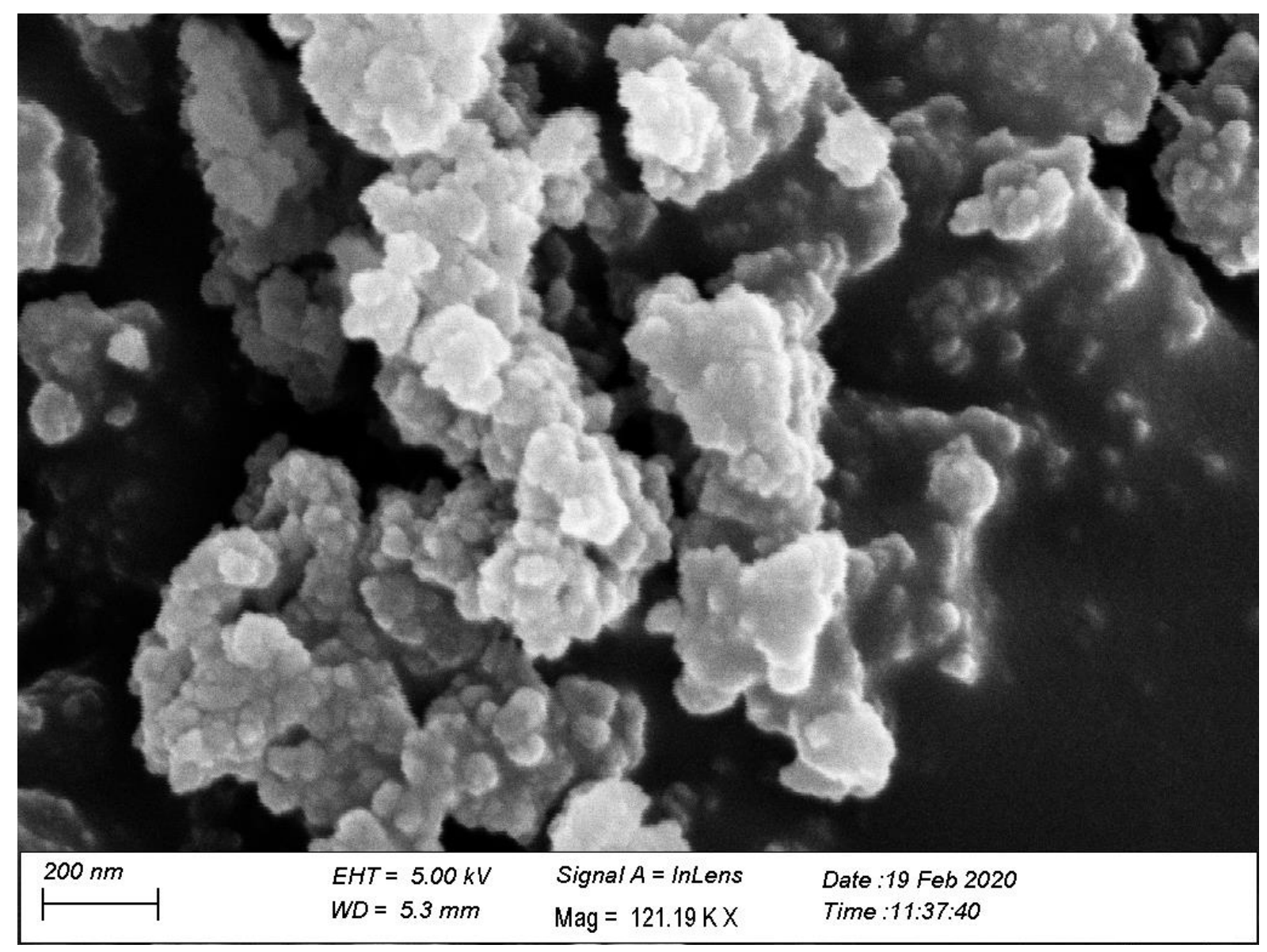

Figure S19. SEM image showing morphology of the humins formed in [bmim] $\left[\mathrm{HSO}_{4}\right]$ 


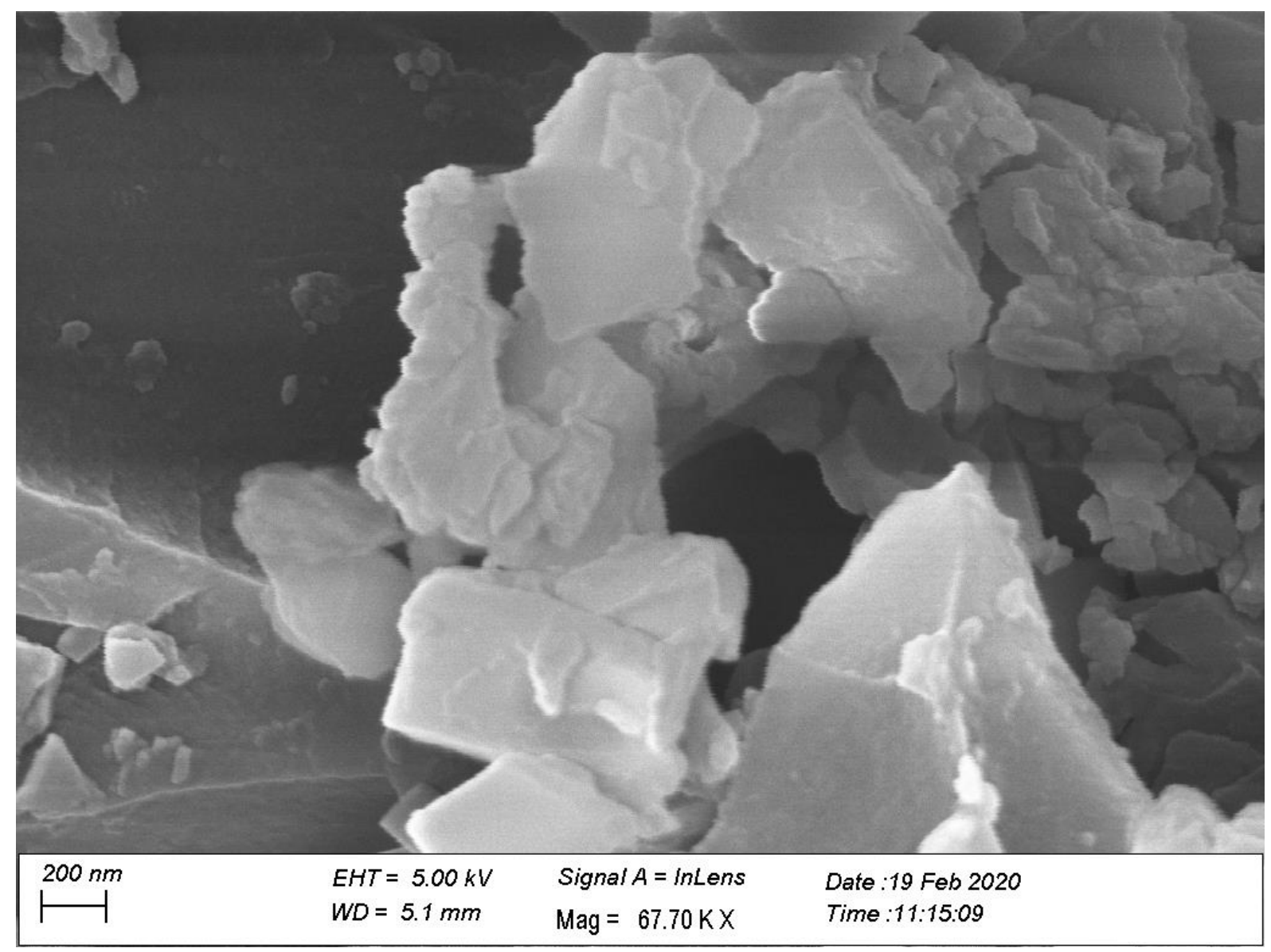

Figure S20. SEM image showing morphology of the humins formed in [bmim]Br 


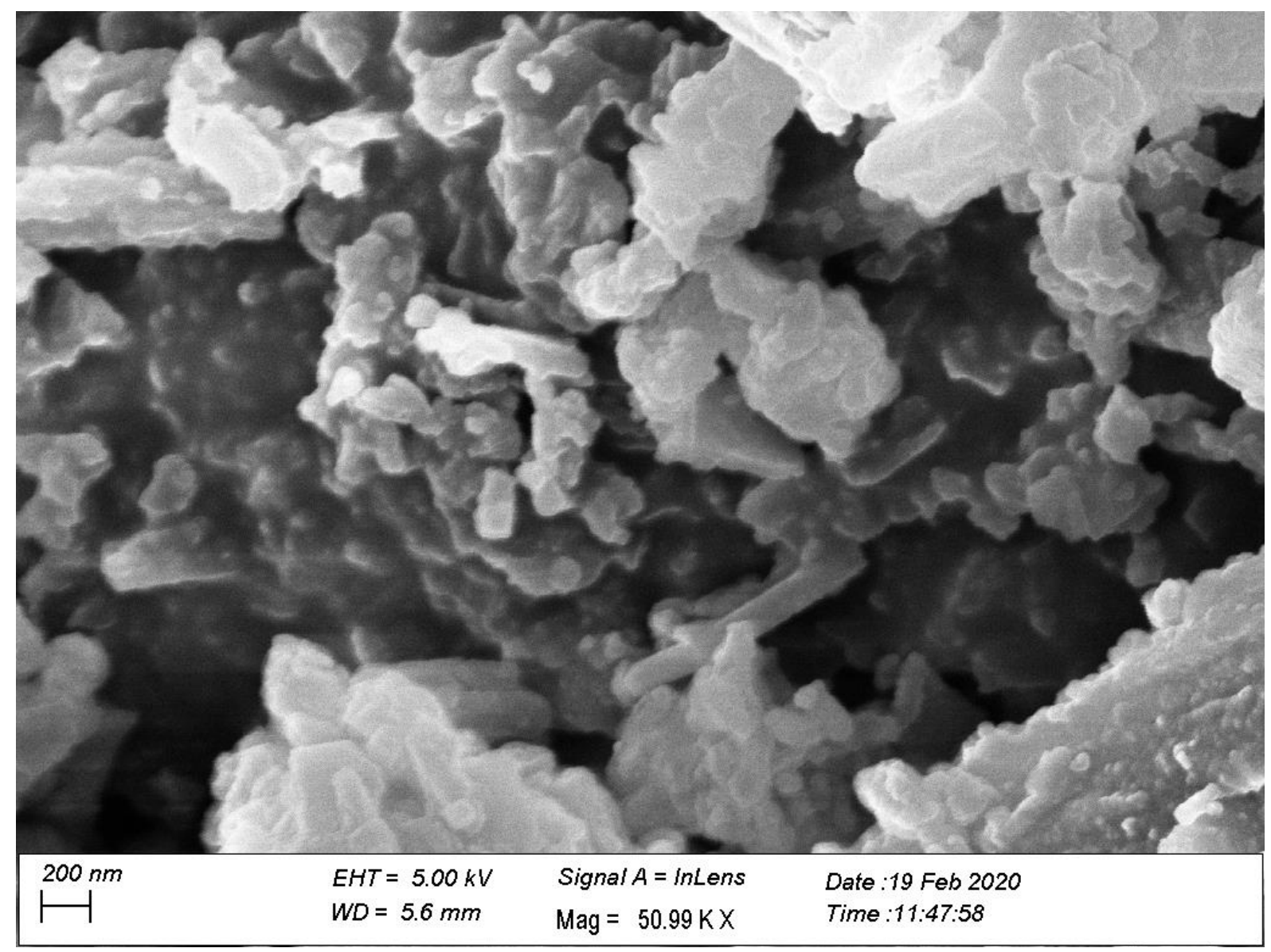

Figure S21. SEM image showing morphology of the humins formed in [bmim][OTf]. 
S6 X-ray Photoelectron Spectroscopy (XPS)

Table S1. XPS data for Humins formed in [bmim][OTf].

\begin{tabular}{lcc}
\hline \multicolumn{1}{c}{ Assignment } & Position (eV) & FWHM \\
\hline C-O (ether) & 286.07 & 1.56 \\
C=O (aldehyde) & 287.54 & 1.80 \\
O-C=O (carboxylic acid) & 289.46 & 1.80 \\
C=C & 284.50 & 1.47 \\
\hline
\end{tabular}

$\mathrm{FWHM}=$ full width at half maximum

Table S2. XPS data for Humins formed in [bmim] $\left[\mathrm{HSO}_{4}\right]$.

\begin{tabular}{lcc}
\hline \multicolumn{1}{c}{ Assignment } & Position (eV) & FWHM \\
\hline C-O (ether) & 286.00 & 1.7 \\
C=O (aldehyde) & 287.91 & 1.75 \\
O-C=O (carboxylic acid) & 288.49 & 1.8 \\
C=C & 284.5 & 1.4 \\
\hline
\end{tabular}

FWHM = full width at half maximum

\section{Freundlich fitting for antimony adsorption}

Table S3. Freundlich fittings for antimony adsorption. The parameters are affected by a degree of error since a perfect plateau was not reached.

\begin{tabular}{llll}
\hline \multicolumn{1}{c}{ Material } & \multicolumn{1}{c}{$\mathbf{Q}_{\text {sat }}$} & \multicolumn{1}{c}{ K } & \multicolumn{1}{c}{$\mathbf{n}$} \\
\hline Activated carbon & $214 \pm 46$ & $7.29 \times 10^{-5} \pm 7.17 \times 10^{-5}$ & $1.89 \pm 0.28$ \\
Humins formed in [bmim][OTf] & $341 \pm 78$ & $1.28 \times 10^{-4} \pm 1.01 \times 10^{-4}$ & $1.71 \pm 0.26$ \\
Humins formed in [bmim] $\left[\mathrm{HSO}_{4}\right]$ & $>500$ & $6.12 \times 10^{-5} \pm 1.10 \times 10^{-4}$ & $1.01 \pm 0.014$ \\
\hline
\end{tabular}




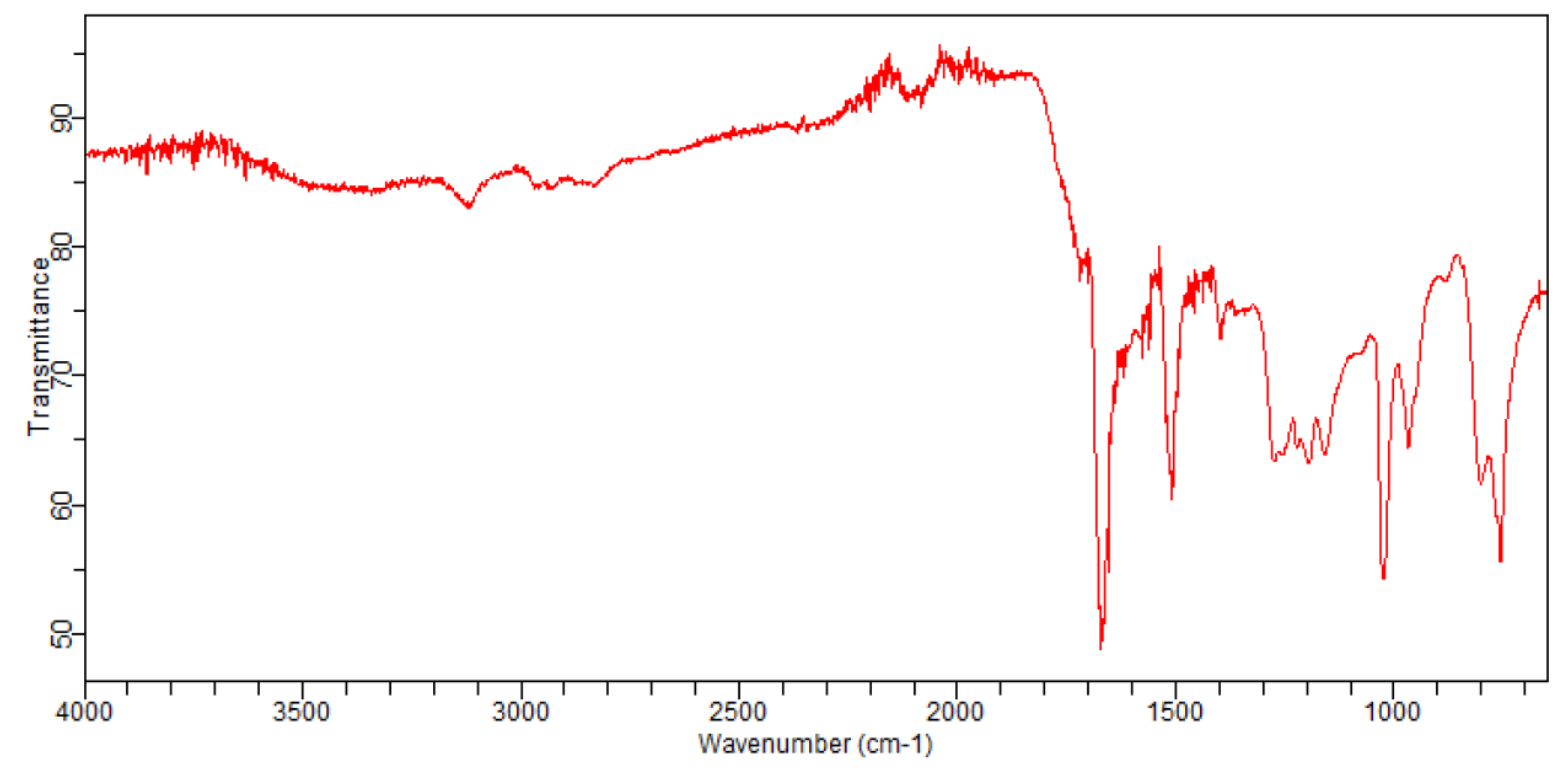

Figure S22. FT-IR spectrum of humins derived from [bmim][OTf] after washing with ethanol.

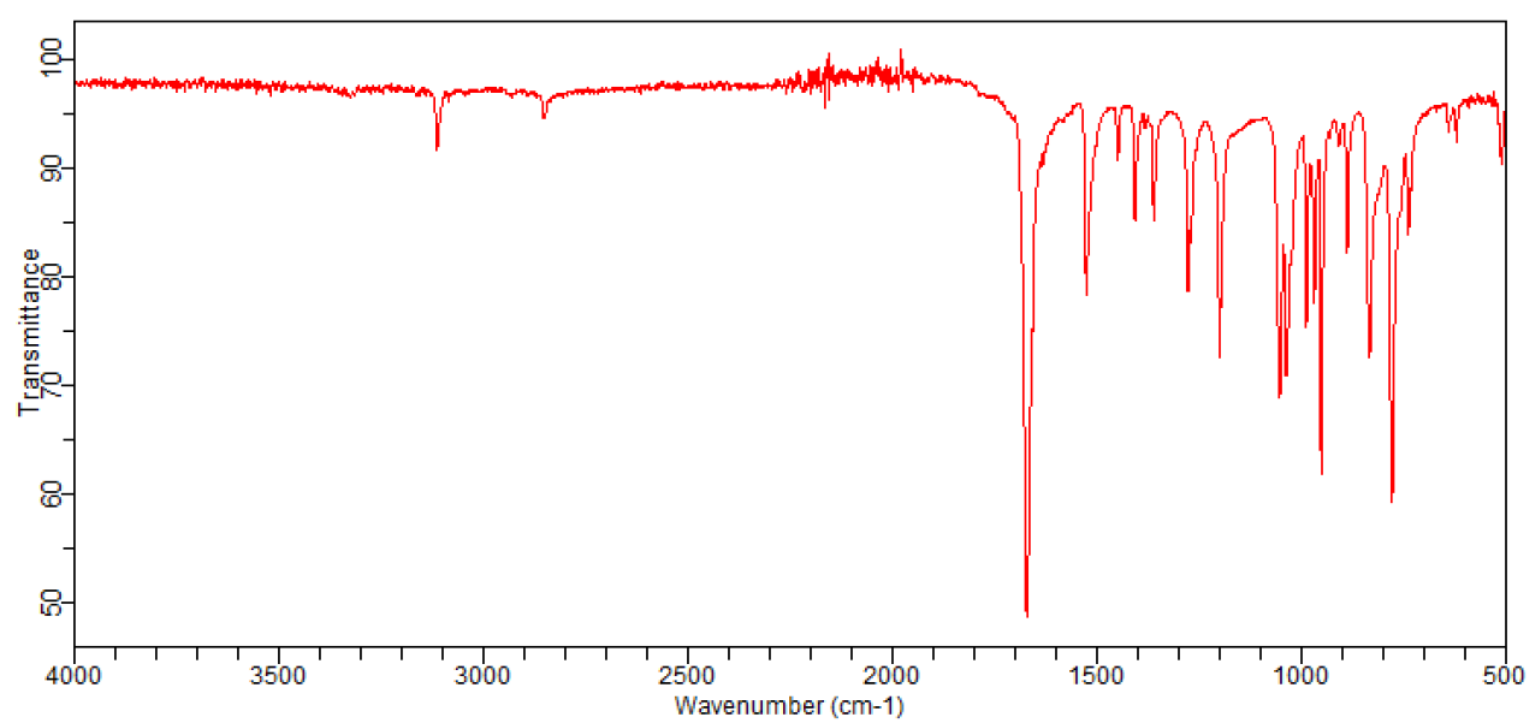

Figure S23. FT-IR spectrum of humins derived from [bmim][OTf] before washing 


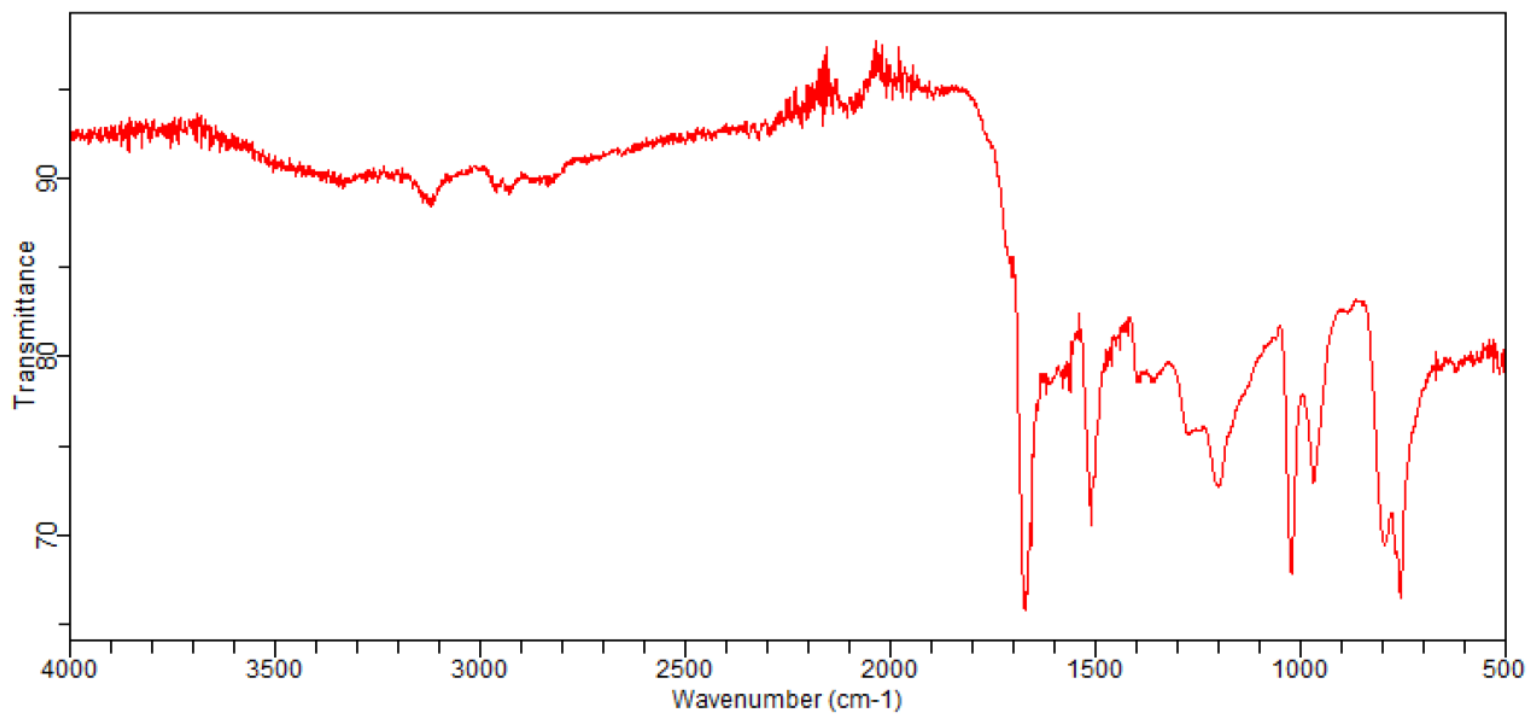

Figure S24. FT-IR spectrum of the humins formed in [bmim] $\left[\mathrm{HSO}_{4}\right]$.

Table S4. IR frequencies and suggested vibrational assignments for humins formed in [bmim] $\left[\mathrm{HSO}_{4}\right]$ and $[\mathrm{bmim}] \mathrm{Br}$. Sym. = symmetrical.

\begin{tabular}{|c|c|c|c|}
\hline \multicolumn{2}{|c|}{ Humins formed in [bmim] $\left[\mathrm{HSO}_{4}\right]$} & \multicolumn{2}{|c|}{ Humins formed in [bmim] Br } \\
\hline Absorption $\left(\mathrm{cm}^{-1}\right)$ & Assignment & Absorption $\left(\mathrm{cm}^{-1}\right)$ & Assignment \\
\hline $3120-2950$ & $\mathrm{sp}^{3}$-like $\mathrm{C}-\mathrm{H}$ stretches & $3110-2870$ & $\mathrm{sp}^{3}$-like $\mathrm{C}-\mathrm{H}$ stretches \\
\hline 1670 & $\mathrm{C}=\mathrm{O}$ stretch & 1664 & $\mathrm{C}=\mathrm{O}$ stretch \\
\hline 1523 & $\mathrm{C}=\mathrm{C}$ sym. stretches & 1523 & $\mathrm{C}=\mathrm{C}$ sym. stretches \\
\hline 1390 & $\mathrm{sp}^{3}$-like $\mathrm{C}-\mathrm{H}$ wagging & 1341 & $\mathrm{O}-\mathrm{H}$ twisting \\
\hline $1277-1192$ & $\begin{array}{l}\text { Multiple rocking and } \\
\text { C-C-C bending }\end{array}$ & $1270-1193$ & $\begin{array}{l}\text { Multiple rocking and } \\
\text { C-C-C bending }\end{array}$ \\
\hline 1020 & $\begin{array}{l}\text { Furanic C-H } \\
\text { Scissoring }\end{array}$ & 1020 & $\begin{array}{l}\text { Furanic } \mathrm{C}-\mathrm{H} \\
\text { scissoring }\end{array}$ \\
\hline 964 & $\begin{array}{c}\text { Furanic } \\
\text { C-O-C sym. stretch }\end{array}$ & 964 & $\begin{array}{c}\text { Furanic } \\
\text { C-O-C sym. stretch }\end{array}$ \\
\hline $755-788$ & $\begin{array}{l}\text { Furanic } \mathrm{C}-\mathrm{H} \text { twisting } \\
\text { and wagging }\end{array}$ & $755-788$ & $\begin{array}{l}\text { Furanic } \mathrm{C}-\mathrm{H} \text { twisting } \\
\text { and wagging }\end{array}$ \\
\hline
\end{tabular}


Table S5. IR frequencies and suggested vibrational assignments for humins formed in [bmim][OTf]. Sym. = symmetrical, Asym. = asymmetrical.

Humins formed in [bmim][OTf]

\begin{tabular}{cc}
\hline Absorption $\left(\mathrm{cm}^{-1}\right)$ & Assignment \\
\hline $3111-2848$ & $\mathrm{sp}^{3}$-like $\mathrm{C}-\mathrm{H}$ stretches \\
\hline 2848 & $\mathrm{sp}^{3}$-like $\mathrm{C}-\mathrm{H}$ stretch \\
\hline 1670 & $\mathrm{C}_{6}=\mathrm{O}_{2}$ stretch \\
\hline 1523 & $\mathrm{C}=\mathrm{C}$ stretch \\
\hline $1447-1359$ & $\mathrm{sp}^{3}$-like $\mathrm{C}-\mathrm{H}$ scissoring and wagging \\
\hline $1276-1198$ & Multiple rocking and $\mathrm{C}_{4}-\mathrm{C}_{5}-\mathrm{C}_{6}$ bending \\
\hline $1051-1037$ & $\mathrm{C}-\mathrm{O}-\mathrm{C}$ asym. stretch \\
\hline 986 & $\mathrm{C}-\mathrm{H}$ rocking out of place \\
\hline 967 & Furanic $\mathrm{C}-\mathrm{O}-\mathrm{C}$ sym. stretch \\
\hline 950 & $\mathrm{sp}{ }^{3}$-like $\mathrm{C}-\mathrm{H}$ rocking \\
\hline 886 & $\mathrm{C}-\mathrm{O}-\mathrm{C}$ sym. stretch \\
\hline $832-777$ & Furanic $\mathrm{C}-\mathrm{H}$ twisting and wagging \\
\hline
\end{tabular}




\section{S8 References}

S1. Eminov, S.; Wilton-Ely, J. D. E. T.; Hallett, J. P. ACS Sustainable Chem. Eng. 2014, 2, 978-981.

S2. Jamehbozorg, B.; Sadeghi, R. J. Chem. Eng. Data 2018, 63, 331-340.

S3. Wells, T. P.; Hallett, J. P.; Williams, C. K.; Welton, T. J. Org. Chem. 2008, 73, 55855588.

S4. Correia, I.; Welton, T. Dalton Trans. 2009, 4115-4121.

S5. Tsurumaki, A.; Ohno, H. Chem. Commun. 2018, 54, $2-9$. 